\title{
Charge-Dipole Acceleration of Polar Gas Molecules towards Charged Nanoparticles: Involvement in Powerful Charge-Induced Catalysis of Heterophase Chemical Reactions and Ball Lightning Phenomenon
}

\author{
Oleg Meshcheryakov \\ Wing Ltd Company, 33 French Boulevard, 65000, Odessa, Ukraine \\ Correspondence should be addressed to Oleg Meshcheryakov, wing99@mail.ru
}

Received 27 July 2010; Revised 6 November 2010; Accepted 10 November 2010

Academic Editor: Donglu Shi

Copyright ( $) 2010$ Oleg Meshcheryakov. This is an open access article distributed under the Creative Commons Attribution License, which permits unrestricted use, distribution, and reproduction in any medium, provided the original work is properly cited.

\begin{abstract}
In humid air, the substantial charge-dipole attraction and electrostatic acceleration of surrounding water vapour molecules towards charged combustible nanoparticles cause intense electrostatic hydration and preferential oxidation of these nanoparticles by electrostatically accelerated polar water vapour molecules rather than nonaccelerated nonpolar oxygen gas molecules. Intense electrostatic hydration of charged combustible nanoparticles converts the nanoparticle's oxide-based shells into the hydroxide-based electrolyte shells, transforming these nanoparticles into reductant/air core-shell nanobatteries, periodically shortcircuited by intraparticle field and thermionic emission. Partially synchronized electron emission breakdowns within trillions of nanoparticles-nanobatteries turn a cloud of charged nanoparticles-nanobatteries into a powerful radiofrequency aerosol generator. Electrostatic oxidative hydration and charge-catalyzed oxidation of charged combustible nanoparticles also contribute to a selfoscillating thermocycling process of evolution and periodic autoignition of inflammable gases near to the nanoparticle's surface. The described effects might be of interest for the improvement of certain nanotechnological heterophase processes and to better understand ball lightning phenomenon.
\end{abstract}

\section{About the Possible Magnitude and Polarity of a Net Electrostatic Charge of Ball Lightning}

Despite numerous attempts, including the most recent ones [1-7], an adequate theoretical and experimental simulation of ball lightning still remains incomplete. At the same time, a simple analysis of the numerous witness descriptions of this phenomenon $[8,9]$ can provide us with useful information, in particular, concerning the possible magnitude and polarity of a net electrostatic charge of lightning balls. Some witnesses described a strong attraction of their hair towards lightning balls flying in immediate proximity to them (at distances of about two-three feet). It is interesting that such a strictly directed attraction of human hair to lightning balls with diameters of $\sim 10-20$ centimetres was repeatedly observed by different witnesses indoors [9]. Our own experience of experimental work, both with highly charged water-based artificial clouds and with megavoltage equipment, shows that an attraction of human hair towards such highly charged objects becomes apparent when an average electrostatic intensity reaches $\sim 1-2 \mathrm{kV} / \mathrm{cm}$. Therefore, in the above cases, one can assume that a potential difference between the visible surfaces of lightning balls and the witnesses' hair could be at least $\sim 60-120 \mathrm{kV}$, and so, a net electrostatic charge of such lightning balls could be at least $\sim 1$ microcoulomb. The most probable polarity of these lightning balls was negative with respect to the grounded witnesses. Several descriptions from other witnesses of ball lightning [9] show that lightning balls can sometimes relatively uniformly and slowly fall from thunderclouds, only appreciably accelerating downwards when approaching the earth's surface. This sudden acceleration, which takes place not far from the 
earth's surface, and the lightning balls' final elongation to the form of an ellipse before they touch the earth's surface, can indirectly indicate that these balls were charged negatively rather than positively-the earth's surface is almost always positively charged with respect to the base of thunderclouds during a thunderstorm.

There are also several detailed descriptions of direct observations of a relatively low-temperature process of ball lightning formation, that is, ball lightning formation without a previous visible stroke of normal lightning $[8,9]$. In particular, such ball lightning formation was repeatedly observed on grounded metal objects, for example, on cast-iron and steel pins of previously destroyed pin-type insulators that were found on pylons of old inoperative electric lines $[8,9]$. During a thunderstorm, these grounded rusty pins could probably generate invisible positive streamers, simultaneously electrostatically spraying the positively charged iron/carbon-based aerosol particles. Such an intense selective corrosion process accompanied by electrospraying of combustible particles from relatively cold corona-forming metal emitters along with a synchronous local generation of a water gas-based reducing atmosphere could be named "fieldassisted metal dusting corrosion" because of its high physical similarity to ordinary metal dusting corrosion processes [1016]. At this point, however, it is only important to note that a cloud of the unipolar charged combustible nano-or microparticles, which are possibly produced in this highvoltage corrosion-electrospraying process, could be a material basis of lightning balls, generated from the grounded conductors during thunderstorms, and probably such lightning balls could be charged positively rather than negatively.

The positive polarity of the charged lightning balls generated from grounded conductors can also explain their typical horizontal flying trajectories at relatively low heights of about $0.5-2$ metres above the ground.

In these cases, the identical polarity of the charged lightning balls and the ground might be responsible for a Coulomb repulsion of these balls from grounded objects. Having analyzed various witness observations, we can assume the following.

(a) The net electrostatic charge of lightning balls can be both positive and negative.

(b) Sometimes lightning balls can be exposed to a partial discharging due to corona or spark discharges from their surface; the discharging processes can perhaps reduce the magnitude of an initial electrostatic charge of lightning balls.

(c) Sometimes a corona discharge from the surface of lightning balls can cause a corona charging of neighbouring low conducting objects with a polarity similar to the polarity of these balls; such a process can cause a subsequent immediate electrostatic repulsion these balls from neighbouring low conducting objects.

(d) A net electrostatic charge of the lightning balls, which attract human hair at distances of about two-three feet, can perhaps reach at least $\sim 1$ microcoulomb. (e) The lightning balls that are frequently described as avoiding contact with grounded conductors and maintaining their approximately constant low flying heights above the ground can be charged with the same polarity as the ground (i.e., positively rather than negatively during thunderstorms), consequently the force of electrostatic repulsion of these balls from the ground and grounded objects at short distances can partially compensate for their weight, contributing to a buoyancy of these balls; a net electrostatic charge of such relatively long-living lightning balls with typical diameters of $\sim 10-20$ centimetres can be much higher than it was supposed in [17]; thus, the magnitude of the net electrostatic charge of lightning balls avoiding contact with grounded conductors can probably also reach at least $\sim 1$ microcoulomb, being in fact limited by a voltage of corona ignition from their surface.

It was repeatedly assumed, for example in $[18,19]$, that the high electrostatic charge of lightning balls could play a major role in the existence of ball lightning. Equally we share this opinion, and in the present paper, we will examine the possible role electrostatic charge plays in the life of ball lightning, still assuming that ball lightning is a cloud of combustible aerosol particles that are exposed to a slow, predominantly electrochemical oxidation [20]. Such a process of the electrochemical oxidation of nano or submicron aerosol particles converts these combustible particles into aerosol batteries - further, for short "nanobatteries" - that are periodically (and perhaps with very high frequencies) short-circuited by intra-particle breakdowns.

According to [20], we can assume the following.

(a) The aerosol particles-batteries can exist either in the form of nano or submicron aggregates, or in the form of nano or submicron core-shell capsules, or in a more realistic combination of these two simplest types, that is, in the form of aerosol aggregates consisting of mixed nano or submicron core-shell particles.

(b) The aerosol particles-batteries can contain at least one reductant component, for example, a metal or carbon-based component, and at least one electrolyte component.

(c) These aerosol particles can use either an internal compact oxidant or external oxidant from ambient air, that is, oxygen gas and/or water vapour.

(d) During the electrochemical oxidation the aerosol particles automatically turn into aggregated or coreshell structured aerosol nanobatteries periodically short-circuited by the intra-particle breakdowns due to both field and thermoionic electron emission taking place within and on the surface of particlesbatteries.

(e) The short-circuited aerosol nanobatteries are free magnetic dipoles, and so they can be exposed to an intense mutual magnetic dipole-dipole attraction, 
forming ball-shaped clouds with high magnetic polarizability.

(f) The non-short-circuited aerosol nanobatteries are free electric dipoles with substantial electric dipole moments, and so they can be exposed to an intense mutual electric dipole-dipole attraction, forming ball-shaped self-assembling clouds with high electric polarizability (Figure 1).

(g) The repeating processes of the short circuits within the separate aerosol nanobatteries can be partially or totally synchronized within a ball lightning cloud; such repeating synchronized collective short circuits of trillions of nanobatteries can generate powerful electromagnetic radiation, which could explain the repeatedly observed cases of red heat of incandescent bulbs' filaments switched off from power sources; such a temporary red heat of the filaments repeatedly was distantly induced during the slow flying of ball lightning at distances of about one-two feet from the switched off bulbs [9].

When discussing the simplest possible processes of spontaneous formation of the short-circuited aerosol nanobatteries from combustible aerosol nanoparticles in a storm atmosphere, it was assumed in [20] that a high concentration of water vapour in the air can significantly modify the mechanism of oxidation of many metal aerosol nanoparticles, converting a normal process of direct oxidation of these nanoparticles by neutral oxidizing species into the predominantly electrochemical, that is, ion-mediated oxidation process in this highly humid atmosphere. During such an electrochemical oxidation, water vapour from humid air contributes to the formation of the hydrated, hydroxide or oxyhydroxide-based, thermolabile porous layers on the surface of metal nanoparticles rather than contributing to the formation of more thermostable layers of mixed anhydrous metal oxides [19], normally growing on the surface of nanoparticles either at very high, dehydrating temperatures or only in dry oxygen [21-26].

In humid air, a quick diffusion transfer of ions can take place through the hydroxide-based electrolyte layers growing on the surface of the nanoparticles due to their oxidation by water vapour. The quick processes of the diffusion of ionized oxidizing species, such as ions $\mathrm{OH}^{-}, \mathrm{O}_{2}{ }^{-}, \mathrm{CO}_{3}{ }^{2-}$, that easily migrate through the dynamic surface electrolyte layers of the mixed hydroxides towards the metal core of the nanoparticle, can considerably prevail above the much slower alternative processes of the inward diffusion of neutral oxidizing species, such as molecular $\mathrm{O}_{2}, \mathrm{H}_{2} \mathrm{O}, \mathrm{CO}_{2}$, or neutral radicals like $\mathrm{OH}$.

Similarly, the quick processes of the diffusion of ions of metal core, such as $\mathrm{Fe}^{2+}, \mathrm{Fe}^{3+}, \mathrm{Fe}(\mathrm{OH})^{+}$, or $\mathrm{Fe}(\mathrm{OH})^{2+}$, to the nanoparticle surface also can significantly prevail above much slower alternative processes of diffusion of neutral atoms from the metal core to the surface of the nanoparticle.

Thus, at relatively low temperatures, while electrolyte layers growing on the surface of metal nanoparticles remain thermostable, a process of the water vapour -induced electrochemical oxidation of combustible nanoparticles can become a most preferable process of their oxidation in humid air because of the possibility of the quick intra-particle transport of ions, and particularly because of the possibility of the quick transport of ionized oxidizing species through the hydrated surface electrolyte layers towards oxidable cores of the combustible nanoparticles.

It seems that in addition to high air humidity another extremely important complementary condition is necessary in order that the process of electrochemical oxidation of combustible aerosol nanoparticles can substantially prevail over the alternative process of their normal oxidation by neutral oxidizing species from ambient air. We suppose that such a complementary condition, radically changing the process of oxidation of combustible nanoparticles in humid air, can be a high electrostatic charge of these nanoparticles. Further it will be shown how and why a presence of electrostatic charges on combustible aerosol nanoparticles can make an important contribution to their preferential oxidation by water vapour molecules but not by much more numerous oxygen gas molecules in humid air that is, how and why electrostatic charges distributed on the surface of combustible aerosol nanoparticles can become powerful selective catalysts of water vapour-induced oxidation of these nanoparticles.

\section{Electrostatic Hydration of Atmospheric Ions and Charged Aerosol Nanoparticles}

As is well known, in normal, humid air, intense chargedipole interaction between atmospheric ions and highly polar molecules of water vapour causes immediate hydration of the ions. A hydrated ion includes a central ion and a water shell normally consisting of several $\mathrm{H}_{2} \mathrm{O}$ molecules. The hydrated ions are extremely stable because of the huge electrostatic energy which keeps polar water molecules in immediate proximity to the central ion (the typical energies of complete dehydration of atmospheric ions can be $\sim$ several electron-volts). Therefore the hydrated ions are a standard form of gaseous ions in the lower troposphere $[8,18,27]$. The charge-dipole interaction between gaseous ions and surrounding polar gas molecules is a powerful and long-range attraction, and intense hydration of gas ions caused by the ion-dipole attraction finds useful applications, for example for an effective operation of the Wilson chamber where gaseous ions generated by ionizing radiation act as condensation nuclei in order to visualize tracks of ionizing particles. In this well known case, hydrated or alcoholsolvated gaseous ions quickly turn into water or alcohol based charged nanoparticles. These charged nanoparticles quickly grow into micrometre-sized droplets due to their further intense electrostatic hydration/solvation under supersaturation conditions.

Electrostatically charged aerosol nanoparticles, especially "small" nanoparticles with characteristic sizes of about several nanometres, almost do not differ from gaseous ions in their ability to attract surrounding polar gas molecules, including water vapour molecules, from ambient atmosphere due to the powerful long-range charge-dipole interaction. In humid air, a surplus electrostatic charge of such nanoparticles is almost always localized to trapping 


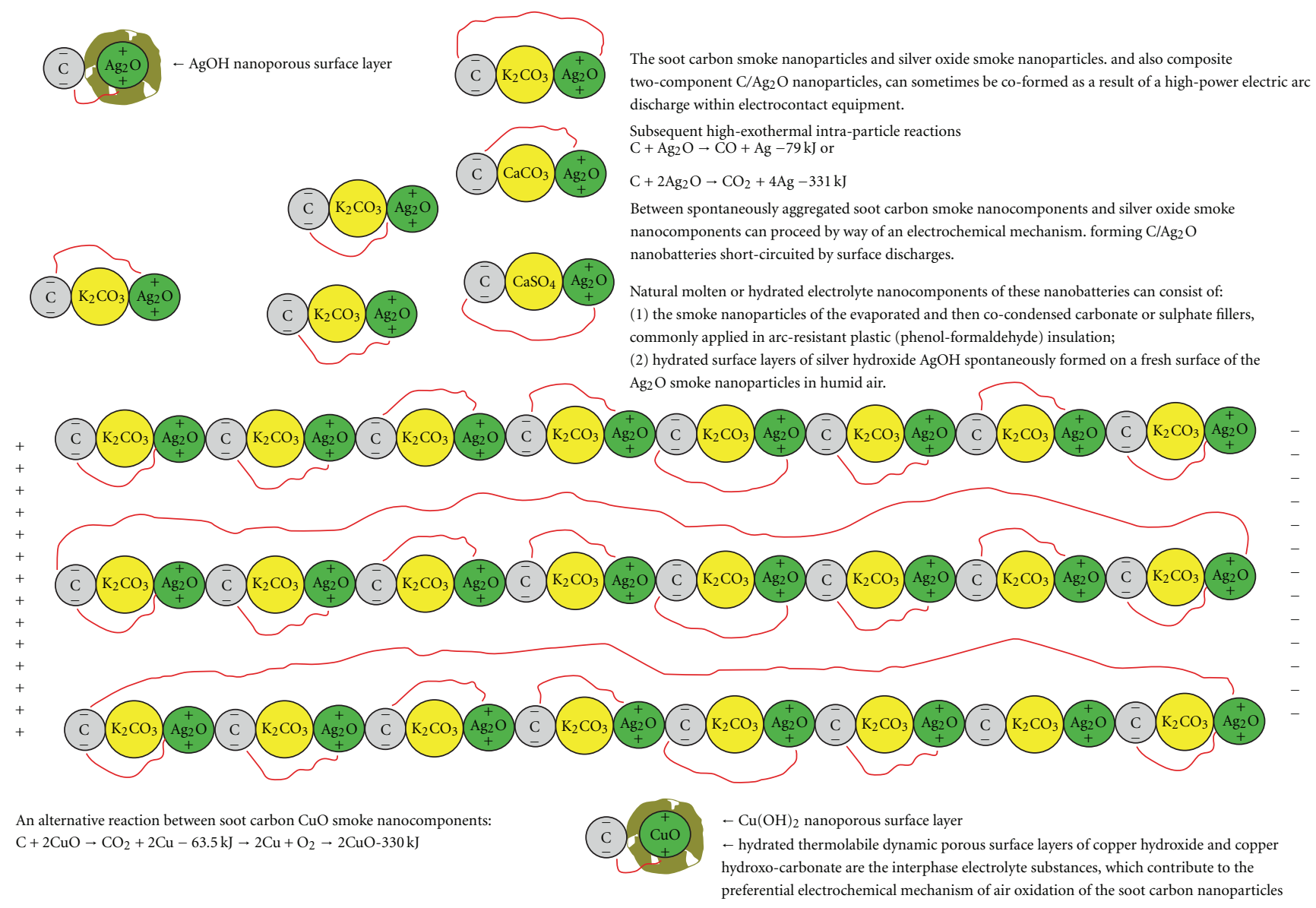

FIGURE 1: electrostatic fields and the high initial concentration of aggregated aerosol nanoparticles-nanobatteries facilitate electrostatic aggregation of individual nanobatteries and automatic conversion of these composite aerosol aggregates to microscopic nanoparticlebased high-voltage generators. Ball lightning can contain some hundreds of billions of individual nanobatteries per cubic centimetre. Every such individual nanobattery is an electric dipole and every short-circuited individual nanobattery is also a magnetic dipole. If the initial concentration of the aerosol nanobatteries is high enough, these nanobatteries can spontaneously form metastable micrometre-sized aerosol chains aggregates due to the mutual electrostatic dipole-dipole attraction. However, an external electrostatic field, for example a thunderstorm electrostatic field, can modify this self-assembling process. The electrostatic aggregation of the nanobatteries enables the periodic formation of relatively long electric circuits consisting of many nanobatteries temporarily connected in series. The micrometresized chain aggregates, which contain only ten thousand individual particles-nanobatteries, can locally generate a ten kilovolt voltage with corresponding macroscopic spark discharges. In particular, the aggregated composite aerosol nanobatteries can be spontaneously formed from soot aerosol nanoparticles-nanoanodes and metal oxide oxidant nanoparticles-nanocathodes. Unipolar charged aggregated aerosol nanobatteries consisting, for example, of the soot carbon-reductant nanoparticles and $\mathrm{CuO}$ oxidant nanoparticles (or also of the soot carbon reductant nanoparticles and $\mathrm{Ag}_{2} \mathrm{O}$ or $\mathrm{PdO}$ oxidant nanoparticles) could be one of the most probable components of lightning balls frequently generated from electrical equipment during thunderstorms.

sites on the nanoparticles surface (in the form of either one or several adsorbed hydrated ions), that is, very close to surrounding polar gas molecules.

If the charged nanoparticles are conducting, surplus electrostatic charges can migrate around their surface in a random manner. In addition, both the conducting and nonconducting charged aerosol nanoparticles can freely and irregularly revolve on their axes due to continuous stochastic Brownian collisions with surrounding gas molecules. In this case, a time-average density of surplus electrostatic charge on the nanoparticle surface can be practically equivalent to the time-average charge density on the surface of the same charged nanoparticle whose surplus electrostatic charge is localized in its centre that is, the surplus electrostatic charge is quasidistributed on the surface of the nanoparticle.

One way or another, it is very likely that the surplus charges distributed on the surface of the hydrophobic or hydrophilic nanoparticles will make a major contribution to their local or total surface hydration in humid air due to the powerful charge-dipole attraction of surrounding polar molecules of water vapour, which is very similar to the hydration of atmospheric ions mentioned above.

In fact, in this case the charge-dipole attraction of water vapour molecules towards the charged surface of the 
nanoparticle plays the role of a powerful electrostatic water pump.

If the charged nanoparticle is cold enough, the electroadsorbed water molecules can strongly hold on to the cold charged surface of the nanoparticle.

If the charged nanoparticle is heated to a high enough temperature, the water vapour molecules previously electroadsorbed on the charged surface of this nanoparticle can be thermally desorbed when heating.

If the electroadsorbed water molecules are not consumed on the surface of the cold charged nanoparticle, for example due to some possible surface reactions, the surface of the charged nanoparticle will remain highly hydrated till the nanoparticle is cold enough.

If the charged nanoparticle consists of a substance that can be oxidized by water vapour at a given temperature, the water vapour-induced oxidative reactions will inevitably take place on the surface of such a charged nanoparticle.

In many cases, during water vapour-induced oxidation of combustible nanoparticles, for example metal nanoparticles, a complete cascade of the primary and secondary oxidative reactions accompanying the water vapour-induced nanoparticle oxidation can be highly exothermal, and so, because of the series of such highly exothermal oxidative reactions, a temperature of the charged "electrohydrated" nanoparticle will grow to some limiting value, while the electroadsorbed water vapour molecules will be subject to thermal desorption from the heated nanoparticle surface, despite the continuous functioning of the "electrostatic water pump". The thermal desorption of the water molecules will reduce the rate of the water vapour-induced oxidative surface reactions, consequently the charged nanoparticle will quickly cool, and so the process of the electrostatic, charge-dipole adsorption of the water vapour molecules will recommence. Again, the rate of the exothermal oxidative reactions will grow, and it will again cause the growth of temperature of the charged nanoparticle and so on. This cyclical oxidative process will repeat until the combustible charged nanoparticle is completely oxidized. Probably a frequency of such a self-oscillating oxidative process might be very high, as possible rates of heating/cooling of the nanoparticles are extremely high.

Clearly, a balance between the competing processes of the electrostatic oxidative adsorption of water vapour molecules on the surface of the charged nanoparticle and thermal desorption of water molecules from this surface can be achieved either in a mode of a synchronous running of both these processes, or in the self-oscillating mode of a successive alternation of these processes, or in a combination of these two modes. It seems, however, that the selfoscillating mode of the successive alternation of the processes of the electrostatic oxidative adsorption of the water vapour molecules on the surface of the charged nanoparticle and their thermal desorption from this surface would be one of the most probable modes. The additional reasons for the high probability of such a self-oscillating mode of water vapour induced oxidation of combustible charged nanoparticles will be discussed in the next section.

\section{The Mechanisms and Products of Water Vapour-Induced Oxidation of Combustible Nanoparticles Radically Differ from the Mechanisms and Products of Their Oxidation by Oxygen Gas}

At least two main gas oxidants with substantial partial pressures are available in the air to oxidize combustible aerosol nanoparticles, irrespective of whether these nanoparticles are electrostatically charged, and consequently, they can actively electroadsorb polar molecules of water vapour from ambient air, or these nanoparticles are electrostatically neutral, and consequently, they will be much more indifferent to surrounding water vapour.

These two main atmospheric oxidants are the following:

(a) oxygen gas, $\mathrm{O}_{2}$, with a mole fraction of oxygen molecules in the air at Sea level, $n_{O}, \sim 0.21$ (i.e., $0.21 \mathrm{~mol}$ of oxygen gas per one mol of air),

(b) water vapour, $\mathrm{H}_{2} \mathrm{O}$, with a mole fraction of water molecules, $n_{W}$, ranging in the air at sea level between $\sim 0.002$ (at very low air temperatures, in particular, in Antarctica) to $\sim 0.01-0.02$ (at normal summer temperatures in temperate latitudes), to $\sim 0.03$ air humidity maximum (in the tropics or during summer thunderstorms in temperate latitudes).

In this paper, we will use the term "humid air" for normal air atmosphere, where a mole fraction of molecules of water vapour, $n_{W}$, ranges from 0.01 to $0.03 \mathrm{~mol}$ of water vapour per one mol of air.

Thus, the term "humid air" will be in fact equivalent to the term "normal air", as the humidity of such "humid air" is typical not only for most thunderstorms, but also practically for any summer weather.

In this paper, we also conditionally use the term "combustible nanoparticles", which requires a more precise definition. This term is used by us to denote aerosol nanoparticles or substrate-integrated/substrate-precipitated nanostructures consisting of condensed materials, which are able to be oxidized by surrounding molecules of both oxygen gas, and water vapour, irrespective of the specific mechanism, rate and optimal temperature of such oxygen-gas or water vapour-induced oxidation.

And so the term "combustible nanoparticles" can be, in particular, applied to aerosol nanoparticles or substrateintegrated/substrate-precipitated nanostructures consisting of the overwhelming majority of metals, metalloids, sulfides, hydrides, carbides, phosphides, nitrides, silicides, borides, lower oxides, many organic compounds, particularly unsaturated organic compounds, many polymer and biopolymer structures. A lot of carbon-based nanoparticles, for example soot nanoparticles, fullerenes, or carbon nanotubes can also be considered as "combustible nanoparticles", because they can be theoretically oxidized by both surrounding oxygen gas molecules and/or water vapour molecules. Thus, in this paper, a wide range of nanoparticles will be conditionally considered as "combustible nanoparticles" in the above aspect. 
The mechanisms of oxidation of combustible aerosol nanoparticles by each of the two competing atmospheric oxidants are radically differing. As mentioned above, reactions of the dry oxygen oxidation of many combustible (e.g., metal or metalloid) aerosol nanoparticles can give reaction products in the form of solid or molten layers of mixed oxides, growing on the surface of the nanoparticles during the process of their oxidation. Alternatively, reactions of oxidation of the combustible aerosol nanoparticles by molecules of pure water vapour usually give simultaneously two types of different reaction products:

(a) solid reaction products in the form of the more or less hydrated, more or less thermostable, more or less porous metal hydroxide shells on the surface of the nanoparticles, and in addition,

(b) combustible gases, in particular, hydrogen gas, when oxidizing the nanoparticles of the great number of reactive metals or metalloids, for example, when oxidizing the nanoparticles of such different substances such as aluminium, iron, tungsten, molybdenum, zirconium, calcium, cadmium, and silicon

As an example, let us compare the reaction products synthesized when oxidizing the silicon based aerosol nanoparticles either in dry oxygen, or in pure water vapour, or in humid air.

Aerosol nanoparticles that consist of pure silicon can be oxidized in dry oxygen to generate nanolayers of the mixed silicon oxides growing on their surface

$$
\begin{aligned}
& \mathrm{Si}_{\text {(nanoparticle core) }}+\mathrm{O}_{2} \\
& \quad=\mathrm{SiO}_{2_{\text {(surface nanolayer }}}(\text { dielectric }) . \\
& 2 \mathrm{Si}_{\text {(nanoparticle core) }}+\mathrm{O}_{2} \\
& \quad=2 \mathrm{SiO}_{(\text {surface nanolayer })} \text { (volatile combustible dielectric) }
\end{aligned}
$$

At high temperatures $\left(\sim 700-1300^{\circ} \mathrm{C}\right)$, either in pure water vapour or in humid air the silicon aerosol nanoparticles can be oxidized to generate growing surface layers of the mixed dielectric silicon oxides plus the evolved hydrogen gas

$$
\begin{aligned}
& \mathrm{Si}_{\text {(nanoparticle core) }}+2 \mathrm{H}_{2} \mathrm{O}_{\text {(vapour) }} \\
& \quad=\mathrm{SiO}_{2 \text { (surface nanolayer) }}(\text { dielectric) })+2 \mathrm{H}_{2} \uparrow, \\
& \mathrm{SiO}_{\text {(surface nanolayer) }}+\mathrm{H}_{2} \mathrm{O}_{\text {(vapour) }} \\
& \quad=\mathrm{SiO}_{2 \text { (surface nanolayer) }}(\text { dielectric) })+\mathrm{H}_{2} \uparrow .
\end{aligned}
$$

At relatively low temperatures, in humid air products of the water vapour-induced oxidation of silicon nanoparticles can include the evolved hydrogen gas and mixed layers of silicic acids, growing on the nanoparticles surface instead of mixed anhydrous silicon oxides. These dynamic porous surface nanolayers can consist of either metasilicic acid $\mathrm{H}_{2} \mathrm{SiO}_{3}$, orthosilicic acid $\mathrm{H}_{4} \mathrm{SiO}_{4}$, disilicic acid $\mathrm{H}_{2} \mathrm{Si}_{2} \mathrm{O}_{5}$, or pyrosilicic acid $\mathrm{H}_{6} \mathrm{Si}_{2} \mathrm{O}_{7}$, or of the mixed silicic acids. Such hydrated surface nanolayers are water-soluble electrolytes with a relatively low thermal stability and low ionic conduction, which, nevertheless, can probably make some contribution to the process of low temperature electrochemical oxidation of silicon nanoparticles [20]:

$$
\begin{aligned}
& \mathrm{Si}_{\text {(nanoparticle core) }}+\mathrm{H}_{2} \mathrm{O}_{\text {(vapour) }}+\mathrm{O}_{2} \\
& \quad=\mathrm{H}_{2} \mathrm{SiO}_{3} \text { (water-soluble volatile electrolyte), } \\
& \mathrm{SiO}_{2 \text { (surface nanolayer) }}+\mathrm{H}_{2} \mathrm{O}_{\text {(vapour) }} \\
& \quad=\mathrm{H}_{2} \mathrm{SiO}_{3} \text { (water-soluble volatile electrolyte). }
\end{aligned}
$$

Silicon-containing aerosol nanoaggregates, which in addition to nanoparticles of pure silicon contain some mineral nanocomponents, such as co-aggregated nanoparticles of either $\mathrm{NaOH}$, or $\mathrm{KOH}$, or $\mathrm{Ca}(\mathrm{OH})_{2}$, or $\mathrm{Ba}(\mathrm{OH})_{2}$ can be co-condensed from the plasma-evaporated silicate or aluminosilicate minerals in a carbon monoxide reducing local atmosphere, which is almost identical with the process described in [19].

In humid air, products of the water vapour-induced oxidation of such silicon containing aerosol nanoaggregates, can include evolved hydrogen gas and surface nanolayers of thermostable water-soluble and/or molten silicate based electrolytes, automatically forming silicon/air coreshell nanobatteries with silicon core, silicate electrolyte shell and external air oxidant

$$
\begin{aligned}
& \mathrm{Si}_{\text {(nanoparticle) }}+2 \mathrm{NaOH}_{(\text {mineral nanoimpurity })}+\mathrm{H}_{2} \mathrm{O}_{\text {(vapour) }} \\
& \quad=\mathrm{Na}_{2} \mathrm{SiO}_{3}(\text { electrolyte })+2 \mathrm{H}_{2} \uparrow \\
& \mathrm{Si}_{\text {(nanoparticle) }}+2 \mathrm{KOH}_{(\text {mineral nanoimpurity })}+\mathrm{H}_{2} \mathrm{O}_{\text {(vapour) }} \\
& \quad=\mathrm{K}_{2} \mathrm{SiO}_{3}(\text { electrolyte })+2 \mathrm{H}_{2} \uparrow
\end{aligned}
$$

Thus, the described above example of the possible alternative pathways of the dry oxygen and humid air-induced oxidation of the silicon or silicon-based nanoparticles illustrates a simple fact: in humid air, reactions of oxidation of combustible aerosol nanoparticles by water vapour frequently can be accompanied by an evolving of combustible gases, most often hydrogen gas. Such an evolving of combustible gases can take place through the process of water vapour induced oxidation of very different nanoparticles. Naturally, this water vapourinduced gradual evolving of the combustible gases on the nanoparticle surface in turn can be accompanied by their periodical spontaneous ignition in humid air.

\section{Partially Synchronized Collective Processes of Electrostatic Oxidative Hydration of Combustible Nanoparticles, Evolution, and Autolgnition of Combustible Gases and Generation of Electromagnetic Radiation in a Cloud of Unipolar Charged Nanoparticles-Nanobatteries}

Within a small cloud of predominantly unipolar charged red-hot nanoparticles-nanobatteries, that is, within ball 
lightning, there are several potential reasons for the continuous or repeating processes of spontaneous ignition of evolved combustible gases. For example, such a spontaneous ignition of combustible gases can occur due to the repeating collective discharge processes of short circuits taking place within and on the nanoporous surface of the aggregated aerosol nanobatteries. On the other hand, such repeating processes of auto-ignition of the combustible gases can occur due to contact of the evolved gases with a fraction of micrometre-sized permanently red-hot aerosol particles"permanent aerosol igniters"- that in turn were previously heated by the preceding intracloud exothermal oxidative reactions, including for example oxygen gas-induced oxidative reactions; these substantially slowed oxygen-induced oxidative reactions can still proceed in humid air on a relatively low-hydrated surface of a fraction of minimally charged or uncharged particles co-aggregated with highly charged particles in order to form composite charged aerosol aggregates, perhaps constituting ball lightning.

It would be reasonable to assume that if ball lightning really is a cloud of charged combustible particlesnanobatteries subjected to slow, predominantly electrochemical oxidation, this cloud most probably contains an extremely polydisperse ensemble of highly aggregated and variously charged particles. One hypothetical fraction of aerosol particles constituting ball lightning could include nano or submicron particles positively charged with possible charge limits of $\sim 1$ up to $\sim 10$ surplus elementary charges per one nano/submicron particle. Another fraction of aerosol particles, which initially form ball lightning cloud, could include nano or submicron particles negatively charged with the same charge limits. Gradual electrostatic aggregation of the first and second aerosol fractions can result in gradual mutual recombination of opposite charges within ball lightning. As mentioned above, the positive and negative charges are substantially unbalanced in ball lightning, totally generating a net electrostatic charge of ball lightning that can reach $\sim 0.1-1$ microcoulomb. A third hypothetical fraction of combustible aerosol particles constituting ball lightning could contain minimally charged or neutral nano-, submicron, or micrometre-sized aerosol particles, which can be aggregated in the form of chains [19] by both a charge-dipole and dipole-dipole electrostatic interaction. A fourth fraction of aerosol particles constituting ball lightning could include relatively large, micrometresized highly charged particles, which play the role of local electrostatic collectors precipitating numerous surrounding chain aerosol aggregates consisting of minimally charged or polarized nano- and submicron particles. A large surface of such an aerosol collector can be charged with tens or even hundreds of surplus elementary charges. These charges, that is, adsorbed hydrated ions, relatively evenly distributed on the surface of the micrometre-sized particles, are local centres of intense attraction of the surrounding polarized chain aerosol aggregates. Electrostatic precipitation of the oppositely charged chain nanoaggregates radially directed to the surface of the micrometre-sized particles-collectors, can contribute to a formation of the sea-urchin/hedgehog-like structures, such as those described in [6].
Surplus charges, distributed on the surface of the micrometre-sized particles-collectors, are centres of electrostatic attraction not only for surrounding chain aerosol nanoaggregates but also for surrounding polar molecules of water vapour. Therefore, the highly charged surface of such micrometre-sized particles can play the role of a catalytic surface where the combustible nano or submicron particles constituting chain aggregates will consecutively react with electroadsorbed (and locally electrostatically accelerated) molecules of water vapour (Figure 2). In this case, electrostatic charges permanently fixed on the surface of the highly charged particles-collectors in the form of adsorbed ions, such as $\mathrm{H}_{3} \mathrm{O}^{+}\left(\mathrm{H}_{2} \mathrm{O}\right)_{n}, \mathrm{OH}^{-}\left(\mathrm{H}_{2} \mathrm{O}\right)_{n}$ or $\mathrm{O}_{2}{ }^{-}\left(\mathrm{H}_{2} \mathrm{O}\right)_{n}$, can play the role of selective catalysts of local water vapour induced oxidation of surrounding nano- or submicron combustible particles, either oppositely charged or only polarized.

So, during the electrostatic precipitation of the chains of the aggregated nano- and submicron aerosol particles on the charged surface of the large particles collectors, the combustible particles from these chains can be exposed to intense water vapour-induced oxidation, locally catalyzed by the surplus electrostatic charges fixed on this surface. Such a scenario of the water vapour induced oxidation of the minimally charged or even neutral but polarized combustible nanoparticles really could require the presence of the fourth hypothetical fraction consisting of the micrometre-sized highly charged aerosol particles - natural catalytically active electrostatic aerosol collectors of both water vapour and surrounding combustible nanoparticles.

Alternatively, surplus electrostatic charges, capable of catalyzing the water vapour induced oxidation of combustible aerosol nanoparticles, could move along chain aggregates, step-by-step contributing to the consecutive oxidation of nanoparticles within chains. Such consecutive charge-catalyzed water vapour-induced oxidation of aggregated combustible nanoparticles by a stepped shift of surplus charge along the chain of the aggregated nanoparticles could resemble the process of Bickford's fuse combustion (Figures $3,4)$.

Clearly, if characteristic sizes of a combustible particle are of $\sim 10-1000 \mathrm{~nm}$ or greater, unipolar surplus charges (i.e., adsorbed hydrated ions) distributed on the surface of such an aerosol particle can form only local mobile surface spots of extremely high electrostatic intensity.

The charge-dipole interaction will cause intense attraction and local acceleration of the surrounding water vapour molecules to these highly charged surface spots, consequently such intense electrostatic hydration of the charged spots on the surface of a combustible particle will result in active water vapour induced oxidation of these small charged sites of the surface of a combustible particle. Being thermally activated during the oxidative process, the surplus electrostatic charges (adsorbed hydrated ions) can jump on the surface of the heated particle in a random manner. And so, this consecutive electrostatic water vapour induced "charge spot corrosion" of the submicron- or micrometresized particles step-by-step can oxidize even such large charged particles-particularly when their surface is highly hydrophilic and so locally electroadsorbed water vapour 


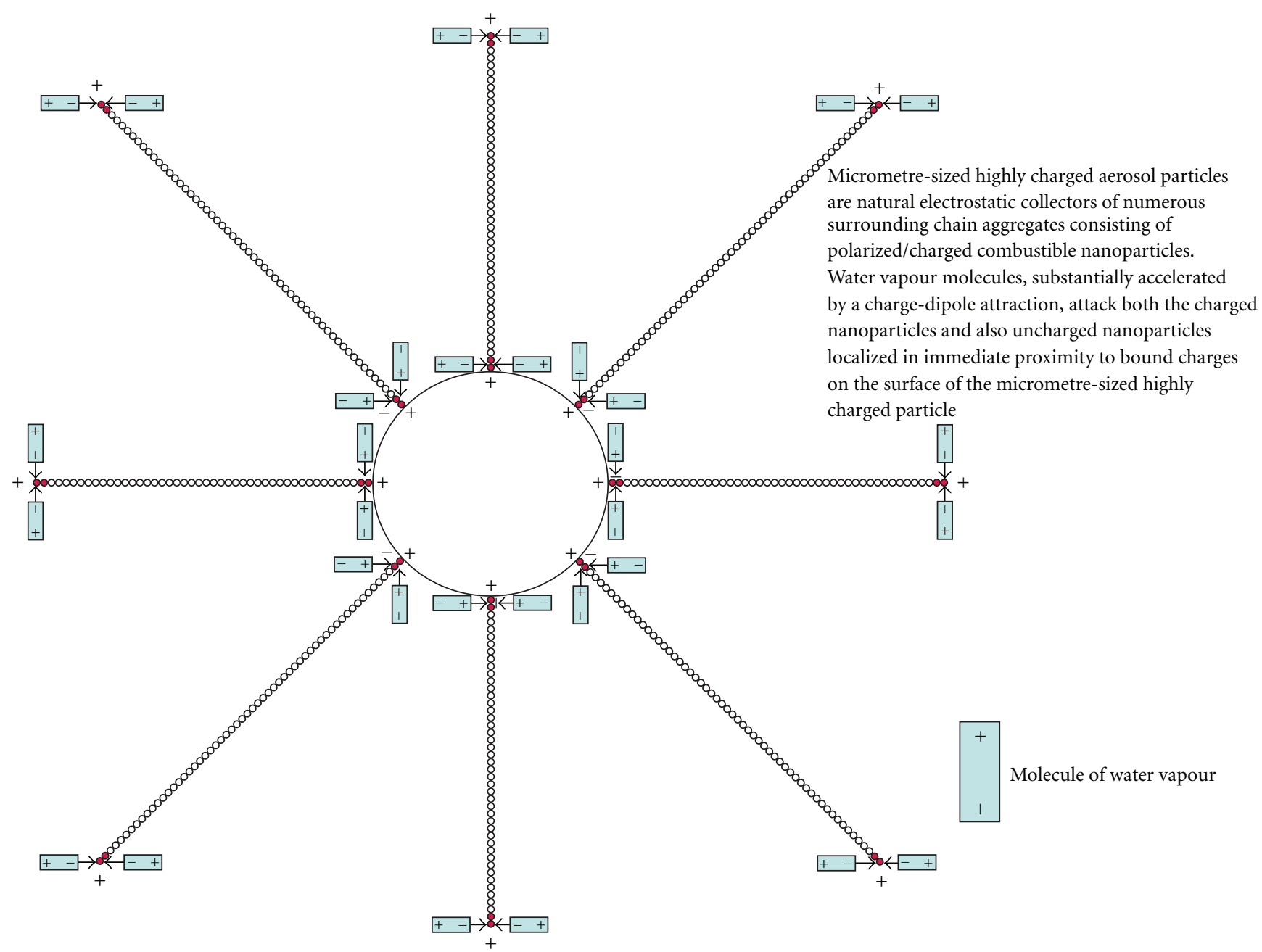

FIGURE 2: Radius of the nanoparticle surface zone, most intensively attacked by surrounding electrostatically accelerated water vapour molecules, can reach $\sim 1-2 \mathrm{~nm}$ in immediate proximity to the site where the surplus charge (i.e., adsorbed hydrated ion) is located at this moment.

molecules can be next distributed on all the surface of the particle by wetting (Figure 5).

Thus, a water vapour induced oxidation of combustible nanoparticles, and particularly the charge-catalyzed water vapour induced oxidation of combustible nanoparticles, is almost always accompanied by an evolution of combustible gases.

Only when a concentration of the evolved combustible gases reaches a lower flammability limit within the nanoparticles cloud, the gases can locally ignite, and a radially spreading deflagration wave can then ignite the whole cloud. Such high-temperature deflagration waves can periodically propagate within the cloud of combustible aerosol nanoparticles and contribute to the periodic thermal dehydration of the electrohydrated nanoparticles. This periodic thermal dehydration of the combustible nanoparticles can temporarily retard their water vapour induced oxidation. However if aggregated combustible nanoparticles constituting this small cloud contain a fraction of charged nanoparticles, the powerful electrostatically induced adsorption of polar molecules of water vapour from ambient humid air will continuously rehydrate these charged nanoparticles, contributing to reactivation of their water vapour induced oxidation, as well as probably to an intra-cloud cyclization and synchronization of the successive processes of electrostatic oxidative hydration and thermal dehydration of the charged combustible nanoparticles.

Each repeating cycle of such intra-cloud collective processes can consist of successive stages:

(a) electrostatic oxidative hydration of the charged combustible nanoparticles accompanied by evolving combustible gases,

(b) autoigniting the evolved gases, for example by their contact with a fraction of the permanently red-hot "igniter" micrometre-sized aerosol particles,

(c) thermal dehydration of the majority of the combustible nanoparticles.

Within a bal lightning cloud, these three-stage cycles can recur repeatedly in the form of a self-oscillating process. 


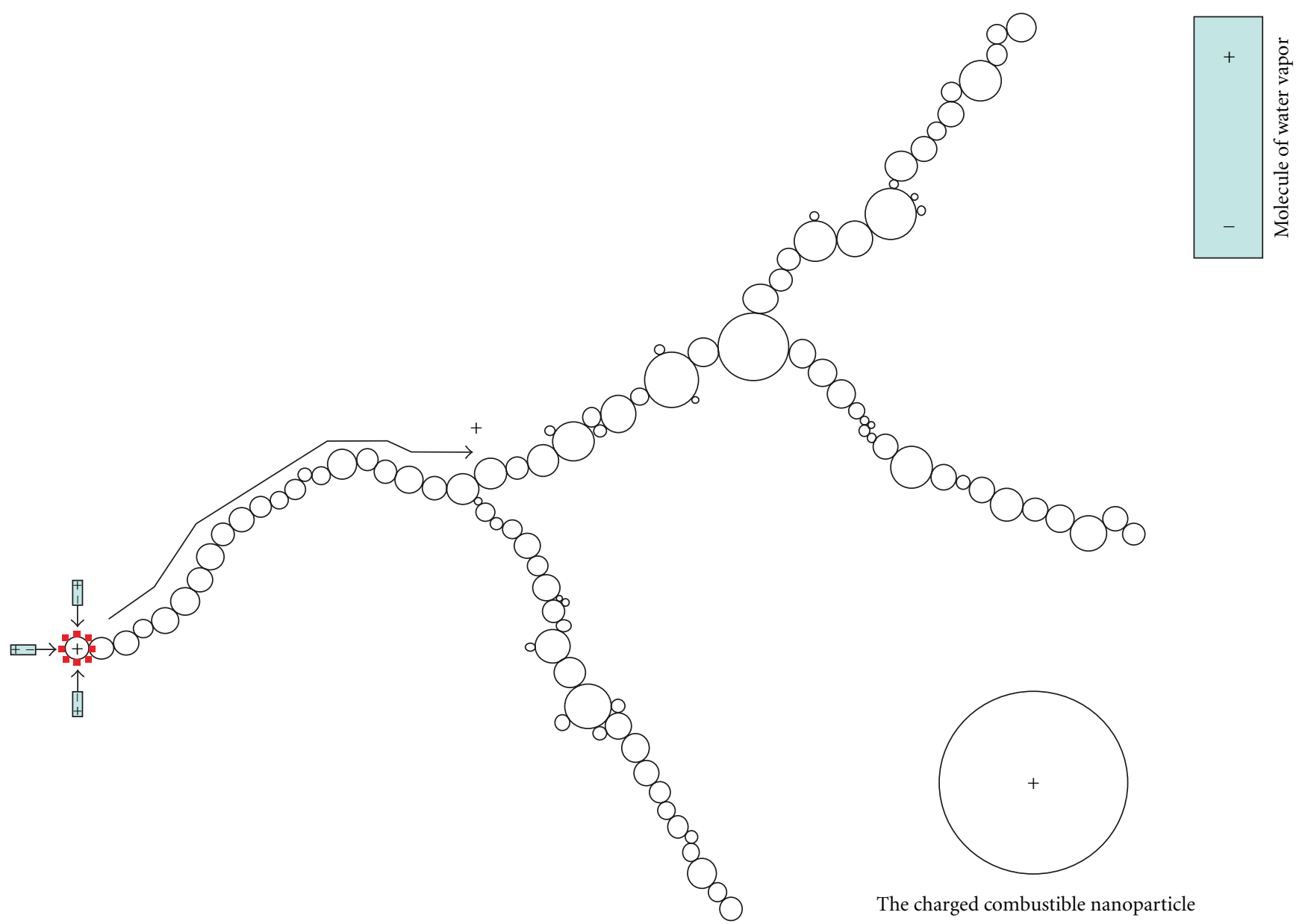

FIGURE 3: Stepped movement of an electrostatic charge (e.g., a hydrated ion) along a chain of combustible aerosol nanoaggregates successively catalyzes water vapour-induced oxidation of these nanoparticles, step-by-step burning the nanoaggregates.

An intra-cloud synchronization of the repeating stages of water vapour-induced oxidation of charged combustible nanoparticles is probably only one of the possible scenarios, but a tendency to such an intra-cloud thermodynamic stage synchronization can arise from at least six interconnected circumstances: (1) relatively slow evolving combustible gases within the ball lightning cloud; (2) relatively fast intra-cloud propagation of the deflagration waves, which consequently can contribute to practically synchronous thermal dehydration of almost all the nanoparticles within the cloud; (3) extremely low thermal inertia of the majority of nanoparticles within the cloud; (4) relatively high thermal inertia of the small fraction of micrometer sized permanently redhot aerosol particles-igniters within the cloud; (5) existence of the lower flammability limits of evolved combustible gases; (6) existence of the relatively prolonged ignition delay time, including ignition hydrogen/air mixtures by highfrequency streamer discharges [28]. If the gas evolved by charged combustible nanoparticles-nanobatteries during the process of their water vapour-induced oxidation is hydrogen, this combustible gas can be periodically autoignited either by collective discharge short circuits within and on the surface of the aerosol particles-nanobatteries or by contact of the hydrogen with the fraction of the large, micrometresized aerosol particles-igniters permanently heated to a temperature higher than $\sim 585^{\circ} \mathrm{C}$, which is the autoignition temperature of air-hydrogen mixtures, or even at a lower temperature-as a result of potential catalytic effects of the highly developed oxidized surface of the heated nanoparticles (such as iron based nanoparticles, which can substantially catalyze the air-hydrogen oxidation reactions).

If the cloud of metal aerosol nanoparticles is exposed to a preferential oxidation by water vapour, the evolving hydrogen can be either autoignited or nonautoignited depending on the concrete local temperature and concentration conditions.

The evolving hydrogen gas can react with ambient atmospheric oxygen both directly on the surface of the metal aerosol nanoparticles and partially in the surrounding gas phase. When the evolving hydrogen is autoignited by oxygen from ambient humid air, we do not see a flame directly, because the air-hydrogen flame is visible only in ultraviolet and not in visual range. However, the ball lightning cloud of electrostatically charged red-hot nanoparticles can play the role of a relatively low-quality natural aerosol visualizer of the air-hydrogen flame generated by this cloud during the 


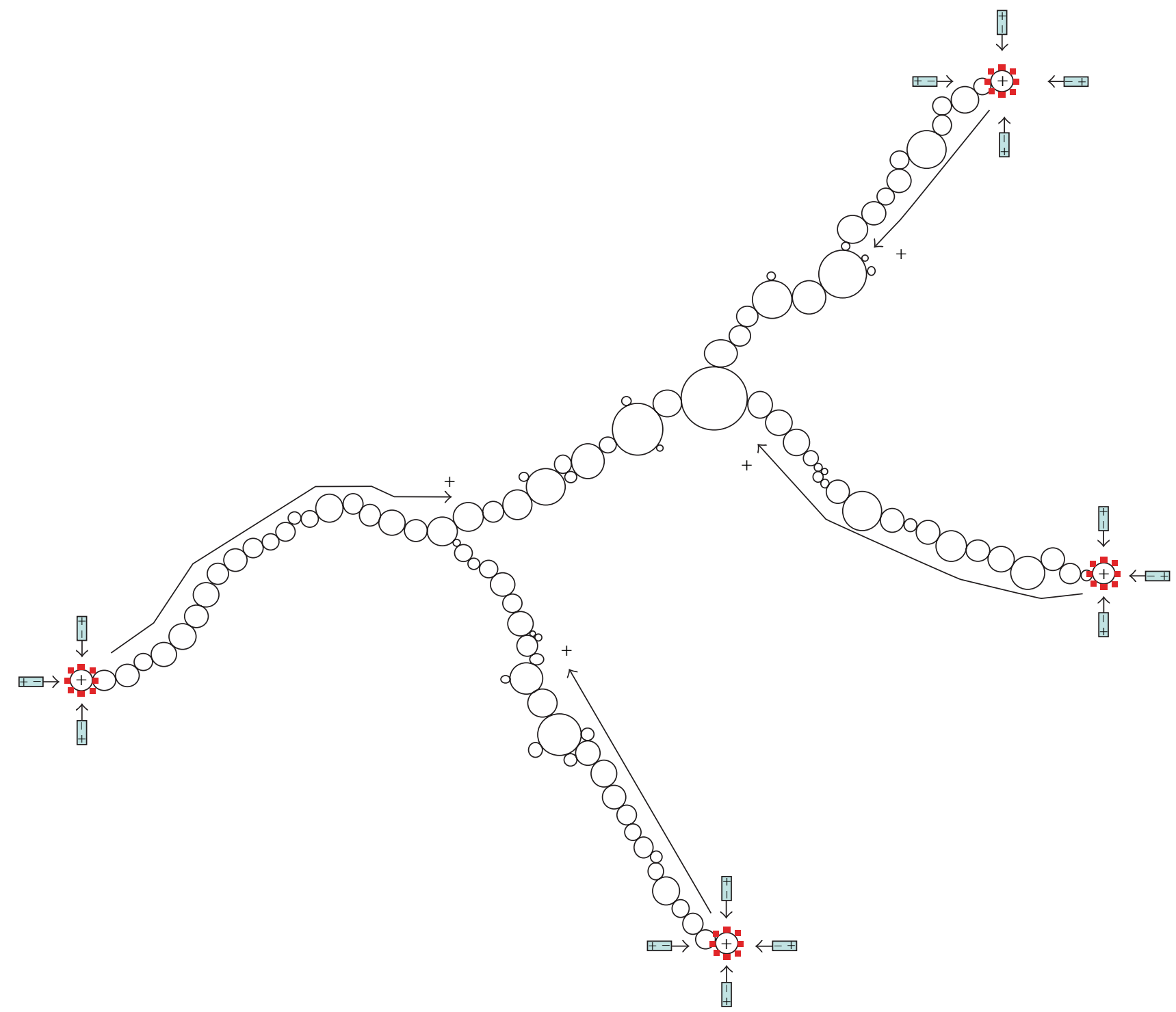

FIGURE 4: Branched chain aggregates of combustible soot or metal nanoparticles are a frequent aerosol product of high-temperature condensation of carbon/metal vapour evaporated by an electric arc. These aggregates can contain a lot of small nanoparticles. Every such aggregate can be charged with several elementary charges. An elementary charge (a hydrated ion) located on a peripheral combustible nanoparticle in the chain is a powerful point catalyst of its oxidation by surrounding polar molecules of water vapour. When the first peripheral nanoparticle is entirely oxidized, the elementary charge jumps onto the next nanoparticle in the chain catalyzing its water vapourinduced oxidation, then the process repeats again, and so forth. During such a charge-catalyzed successive oxidation of nanoparticles in aerosol nanoaggregates, the elementary charge moves along the chain of the oxidizable nanoparticles linked with a wave of water vapourinduced oxidation of the nanoparticles in a similar way to how a flame front moves along a Bickford's fuse. Thus, even a single elementary charge can enable the successive catalytic oxidation of a lot of the small combustible nanoparticles, when moving this charge along the aerosol nanoaggregate. The stepped movement of adsorbed ions along the chains of combustible aggregates successively catalyzes their water vapour induced oxidation, ultimately, completely burning off these aggregated aerosol particles.

process of the preferential water vapour induced oxidation of the charged metal nanoparticles. If the combustible aerosol nanoparticles are strongly interconnected within the cloud by a long-range mutual attraction, for example by the dipole-dipole magnetic attraction, they can form a relatively heavy stable ball-shaped cloud, and so convection currents produced by the air-hydrogen flame cannot appreciably influence both the motion and shape of this heavy stable aerosol cloud [20].

Thus, in this case, we can only see a luminous ballshaped cloud of the distantly interconnected red-hot aerosol nanoparticles, but not an air-hydrogen flame cone.

In addition to hydrogen, the different combustible gases can also be generated through the process of the water 

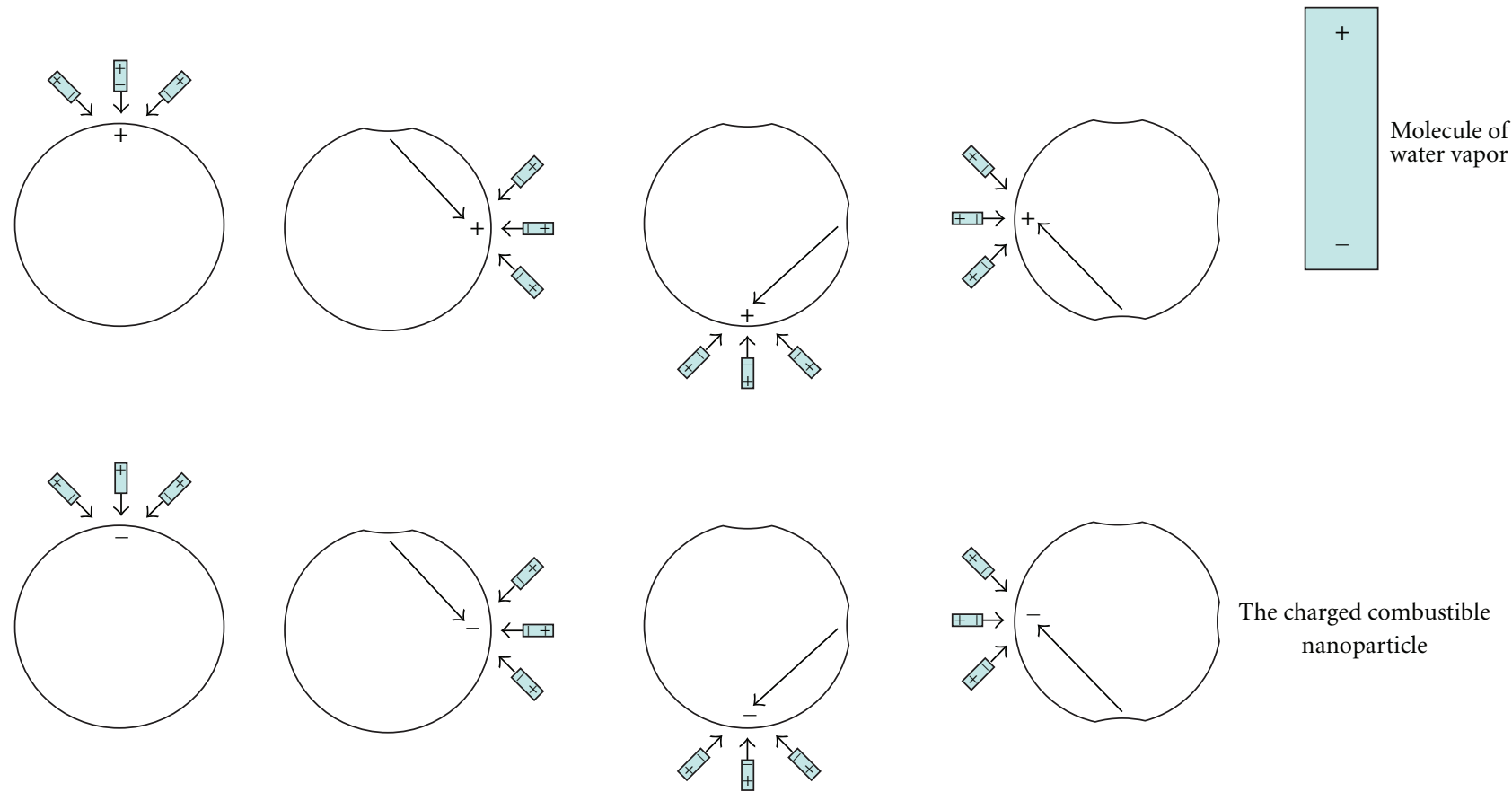

Figure 5: Successive "jumping" of the surplus charge-a local catalyst of water vapour-induced oxidation of combustible nanoparticlebetween the charge trapping sites on the surface of the relatively large (i.e., $\sim 5-100 \mathrm{~nm}$ in diameter) combustible nanoparticle causes numerous successive events of local corrosion of this surface with a final water vapour-induced oxidation of the whole nanoparticle.

vapour-induced oxidation of different combustible aerosol nanoparticles, including nonmetallic ones. For example, a combustible mixture of carbon monoxide and hydrogen, socalled water gas, can be evolved during the process of preferential water vapour-induced oxidation of carbon-based nanoparticles, such as soot nanoparticles, fullerenes, carbon nanotubes, or metal carbide nanoparticles. A completed water vapour induced oxidation of these nanoparticles will generate gas reaction products in the form of carbon dioxide and combustible hydrogen. Alternatively, the completed dry oxygen-induced oxidation of these nanoparticles would generate only noncombustible carbon dioxide. Similarly, a combustible gas mixture of phosphine and hydrogen can be evolved through the process of water vapour-induced oxidation of calcium phosphide aerosol nanoparticles. These nanoparticles can be generated as aerosol products of plasma-chemical reduction of organic calcium phosphate by organic carbon. A formation of such a small cloud of calcium phosphide nanoparticles can, for example, arise when a bird is struck by regular lightning. Similarly, in humid air a combustible hydrogen sulphide can be generated through the process of the water vapour-induced oxidation of many metal sulfide nanoparticles, such as natural particles of weathered iron sulfide minerals and also some organic sulfide nanoparticles. Alternatively, a completed dry oxygen-induced oxidation of metal sulfide nanoparticles can generate only noncombustible solid metal sulphates, metal oxides, as well as the sulphur dioxide and sulphur trioxide gases. Thus, as one can see the mechanisms and products of water vapour-induced oxidation of many absolutely different combustible nanoparticles radically differ from the mechanisms and products of their oxidation by oxygen gas.

Although a cone of flame is as a rule invisible over ball lightning, in the literature, there are several alternative descriptions of observations of lightning balls combined with tongues of flame emerging from them $[8,9]$. On the one hand, these rare observations can show that sometimes forces of a dipole-dipole attraction between separate aerosol particles-nanobatteries constituting ball lightning are inadequate to keep all the particles inside the ball cloud, and some part of the hot aerosol particles can be captured by ascending convective currents. On the other hand, these rare observations show that sometimes combustible gases, evolved during the process of water vapour-induced oxidation of aerosol particles-batteries constituting ball lightning, contain components, which are able to generate visually distinguishable tongues of combustion products.

In our preliminary experiments, small burning clouds of combustible, ethanol and methanol-based, droplets with the droplets diameters of $\sim 1-3$ micrometres were generated. A flow of these droplets suspended in the air was generated with the help of a simple ultrasonic drug inhalator with an oscillator frequency of $\sim 1$ megahertz. The ethanol and/or methanol-based solutions contained from 2 up to 5 percent by weight of acetyl salicylic acid (99.0\%, Sigma-Aldrich) plus from 2 to 3 percent by weight of calcium nitrate tetrahydrate (98.0\%, Sigma-Aldrich) and plus from 2 to 5 percent by weight of cupric nitrate hemipentahydrate $(98.0 \%$, SigmaAldrich). It is important to note that in these experiments the micrometre-sized droplets of the combustible solutions 
were not exposed to additional artificial electrostatic charging. Only minimal spontaneous bipolar charging of the micrometre-sized combustible droplets took place during their ultrasonic generation.

When burning, the droplets of the ethanol and/or methanol based solutions were subjected to immediate evaporation, initially turning into solid particles of acetyl salicylic acid mixed with calcium nitrate and copper nitrate. These solid aerosol particles in turn were exposed to a thermal oxidative decomposition to form small flame clouds consisting of submicron soot particles impregnated with a combination of calcium nitrate/calcium hydroxide/calcium oxide and copper nitrate/copper hydroxide/copper oxides. Obviously, the final target products of the thermal oxidative decomposition form as aggregated soot-based combustible submicrometre particles consisting of amorphous carbon mixed with the calcium and copper oxides. In addition, some other organic/inorganic solution compositions originally containing nitrates of alkaline metals also were investigated with the purpose of formation of the clouds of such carbon/air aerosol nanobatteries.

Several fragments of video of these almost electrostatically neutral burning small clouds are shown in Figure 6. As one can see, the clouds have a high-temperature stable ball-shaped core and a relatively cold and transparent cone of flame. Diameters of the ball-shaped cores of these small burning clouds were from $\sim 10$ to $35 \mathrm{~mm}$. These ball-shaped flame clouds could be retained as long as the aerosol flow of the micrometre-sized droplets was directed into the zone of combustion. The time of independent life of such ballshaped small flame clouds was only of $\sim 50-120$ milliseconds.

It is necessary to note that video fragments shown in Figure 6 are used here only to experimentally illustrate the possibility of coexistence (and a visual observation) of a ball-shaped cloud (consisting of the burning hot submicron soot particles impregnated with inorganic electrolyte components) and visually distinguishable tongues of flame over this spherical cloud. Thus, the above illustration will not be connected to our further discussion, because burning particles constituting ball-shaped flame clouds shown above were only slightly charged with negligible bipolar charges, and the low bipolar charges of these aerosol particles were probably generated due to:

(1) thermoionic electron emission from the surface of hot particles during combustion,

(2) adsorption of negative gas ions to the surface of relatively cold aerosol particles.

The same type of thermoionic bipolar electrostatic charging of nanoparticles takes place in many processes involving dusty plasma, and probably also in the processes describing microwave-induced formation of ball-shaped dusty plasma in $[5,6]$.

Undoubtedly, due to an intense charge-dipole attraction, in humid air both the positively and negatively charged combustible nanoparticles suspended either in a flame or in low-temperature air dusty plasma can be actively attacked and oxidized by the surrounding highly polar molecules of water vapour rather than by non-polar molecules of oxygen. However, parallel with the nanoparticle charging processes, active synchronous processes of neutralization of the bipolar charges of the flame/plasma suspended particles (caused either by cooling of the particles or by mutual recombination of opposite charges of the co-aggregated bipolar particles) can strongly reduce the final charges of these particles.

Consequently, because of gradual recombination of bipolar charges, within such practically quasineutral, flame or dusty plasma clouds containing overwhelming majority of uncharged or minimally charged combustible nanoparticles, the electrostatic delivery of polar molecules of water vapour to the reactive surface of these hot particles will be significantly slowed down, and so the charge-catalyzed water vapour-induced oxidation of these quasi-neutral aerosol particles will be finally minimized.

Thus, if we wish to accomplish a continuous process of predominantly water vapour-induced oxidation of permanently charged combustible aerosol particles, a surplus, uncompensated electrostatic charge is necessary for a cloud of such particles.

So, as assumed above and as it will be quantitatively proved later, in humid air, electrostatically charged combustible particles can be exposed to preferential oxidation by surrounding polar molecules of water vapour rather than to alternative oxidation by non-polar molecules of oxygen gas due to intense charge-dipole attraction of the polar gas molecules to the charged aerosol particles. During the process of preferential water vapour induced oxidation of the charged aerosol nanoparticles, the evolving and autoigniting of combustible gases, in particular, hydrogen gas, on the one hand, and a synchronous growing of the hydroxide-based dynamic thermolabile electrolyte layers on the surface of these charged nanoparticles, on the other hand, can take place within the cloud of such nanoparticles. Both the surface growth of the hydroxide-based porous electrolyte layers and evolution of the combustible reducing gas on the nanoparticles surface hamper oxidation of the nanoparticles by external neutral oxidizing species, first of all such as molecules $\mathrm{O}_{2}$, but at the same time, the hydrated surface electrolyte layers can effectively transport either metal ions from reductant cores to the nanoparticles surface or negatively charged, ionized oxidizing species from the outer surface of the nanoparticles to their reductant cores, contributing to predominantly electrochemical, that is, ion-mediated oxidation of these combustible nanoparticles. Consequently, the electrostatically charged, periodically electrohydrated combustible aerosol particles, being coaggregated with numerous surrounding polarized neutral particles, spontaneously transform into aerosol nanobatteries periodically short-circuited by the field and thermoionic electron emission from their reductant/metal cores (the electron emitting anodes of these nanobatteries) towards external surfaces of their porous thermolabile electrolyte shells (the air depolarized cathodes of these nanobatteries) [20].

The repeating processes of auto-ignition and combustion of evolved combustible gases can make an important contribution to synchronously repeating processes of heating of 


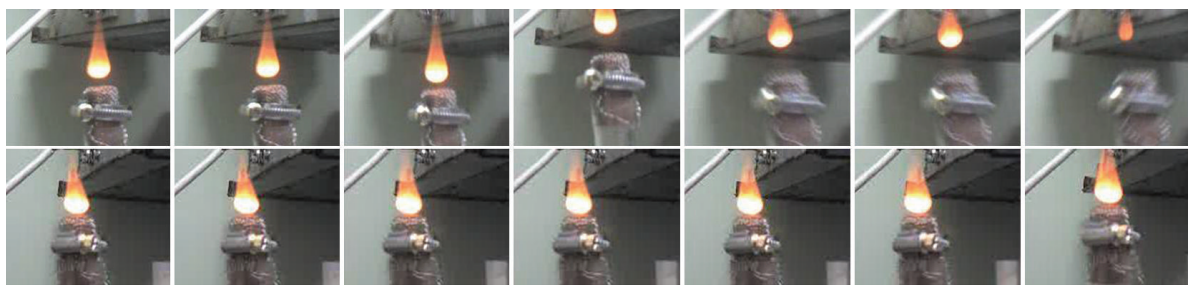

FIgURE 6: Practically neutral burning small clouds with a high-temperature ball-shaped core and a relatively cold and transparent cone of flame consist of billions of submicron soot-based aerosol particles, which can be only minimally charged with almost balanced bipolar charges.

the charged nanoparticles-nanobatteries and consequently to their repeating flame thermal dehydration.

During such "thermocycling," consisting of alternating stages of the electrostatic, charge-dipole hydration of the nanoparticles and their subsequent flame thermal dehydration, electrophysical properties of the interphase contact between a reductant, for example metal core of the nanoparticle-nanobattery and its growing, either metal oxide or metal hydroxide, shell periodically will radically change, with an alternation from the metal-semiconductor (metaldielectric) core-shell junction (in the stage of thermal dehydration of the nanoparticle shell) to the metal-electrolyte core-shell junction (in the stage of electrostatic surface rehydration of the temporarily cooled nanoparticle).

These fast cyclic changes of the electrophysical characteristics of the interphase core-shell contacts will result in cyclic processes of the interphase electron-ion transfer (electronion jumping), forming strong local interphase core-shell electrostatic fields, which in turn will control the specific stage-dependent mechanisms of the nanoparticles oxidation, in particular, from the Cabrera-Mott oxidation mechanismin the stage of flame thermal surface dehydration of the combustible nanoparticle-to the electrochemical oxidation mechanism - in the stage of electrostatic surface rehydration of this nanoparticle $[20,29,30]$.

It is important to note that in the case of both the Cabrera-Mott mechanism of the nanoparticle oxidation and the electrochemical mechanism of its oxidation, each instantaneous event of gradual oxidation of combustible metal/metalloid aerosol nanoparticle will necessarily result in the generation of a powerful instantaneous local coreshell electrostatic field and consequently the generation of an instantaneous electric dipole moment of this nanoparticle.

Clearly, when a combustible aerosol particle is nanoor submicrometre sized, each instantaneous event of its gas phase oxidation from one side cannot be compensated by the same synchronous event of its oxidation from the other side (stochastic nature of such successive spotty oxidation of small aerosol particles is absolutely similar to a locally unbalanced character of Brownian collisions). Therefore, individual nano- or submicrometre combustible aerosol particles will always possess the instantaneous uncompensated electric dipole moments generated during their gas phase oxidation.

The Cabrera-Mott oxidation mechanism assumes primary migration of electrons from a metal core into a nanoporous dielectric or semiconductor metal-oxide shell. In this case, the initial electron migration generates a local electrostatic field between the metal core and the metal oxide shell, and this field further contributes to intense outward electrodiffusion of the metal ions with their following surface oxidation.

The alternative electrochemical mechanism of oxidation assumes primary diffusion migration of metal ions from a metal core into a nanoporous surface electrolyte, for example into a more or less hydrated metal hydroxide shell. In this case, initial outward migration of the metal ions generates a local electrostatic field between the metal core and the metal hydroxide shell, and this field further contributes to intense field/ thermoionic electron emission from the metal core to the outer surface of the nanoparticle, with following surface electrochemical oxidation of the metal ions (naturally, only when these ions recombine with emitted electrons).

In fact, both these mechanisms of oxidation are electrochemical, because diffusion (or electrodiffusion) of ions through porous surface layers is a key process that precedes the events of the metal ion oxidation in both cases.

Both these types of oxidation of metal or metalloid based aerosol nanoparticles can generate powerful momentary electrostatic fields, high instantaneous electric dipole moments and strong instantaneous relaxation electron/ion core-shell currents within the nanoparticles irrespective of whether these nanoparticles are extra charged or not.

Consequently, both these mechanisms of oxidation can convert combustible aerosol nanoparticles into shortcircuited nanobatteries.

It is possible to assume, however, that if a process of the metal/metalloid oxidation takes place in the real, that is, humid air, then a local negative surface charge generated by electrons, which migrate from a metal core into growing metal oxide surface layers (according to the CabreraMott oxidation mechanism) will always cause immediate electrostatic hydration of such highly charged surface sites, locally transforming these surface sites from their purely metal oxide (i.e., dielectric or semi-conductor) state into a hydrated, for example metal-hydroxide (i.e., electrolyte) state.

Thus, it seems that a process of atmospheric oxidation of many metals or metalloids can frequently be automatically converted from the Cabrera-Mott oxidation mode into the true electrochemical mode of oxidation only due to powerful fast spontaneous electrostatic hydration of oxidatively 
charged surface sites, which is inevitable in the conditions of the real, humid air oxidation.

It is important to emphasize that such a predominantly electrochemical mode of atmospheric oxidation of many metal structures, including metal nanoparticles, can arise only owing to natural redistribution of electrons between a metal core and a dielectric or semi-conductor metal oxide surface layer (i.e., without additional electrostatic charging of a metal object exposed to atmospheric oxidation).

Probably, in many cases, within a ball lightning cloud the heat of combustion of evolved combustible gases e.g., hydrogen gas) can considerably exceed the heat that is generated or is consumed in primary heterophase reactions of water vapour-induced oxidation of metal, or metalloid, or carbonbased combustible particles constituting ball lightning.

Thus, the highly endothermic stage of the thermal dehydration of the electrostatically charged combustible nanoparticles can be appreciably delayed in time with respect to the stage of their electrostatic oxidative hydration-only the heat of combustion of the evolved gases can effectively temporarily dehydrate the charged nanoparticles.

So, within ball lightning electrostatically charged combustible nanoparticles can be subjected to preferential oxidation by polar molecules of water vapour rather than nonpolar molecules of oxygen gas. A periodic fast alternating of the processes of electrostatic hydration and thermal dehydration of the charged nanoparticles in humid air can be partially or totally synchronized with the repeating processes of evolution of combustible gases, their auto-ignition, and following quick flame extinction.

Naturally, the fast alternating processes of electrostatic oxidative hydration and thermal dehydration of the charged aerosol particles constituting ball lightning can cause periodic melting and hydrogen gas-induced foaming these particles; formation of submicron or micrometre-sized electrostatically charged metal/metal oxide/metal hydroxide hollow globules can be a natural outcome of such remelting/refoaming.

Many witnesses who observed ball lightning at very short distances described ball lightning as a relatively lowtemperature object, which did not radiate intensive thermal radiation $[8,9]$. Indeed a time-average temperature of the charged nanoparticles can be relatively low owing to a quick alternating of the processes of their intense heating and cooling. At the same time, peak "colour" temperatures of the hot nanoparticles, which are periodically reached in the quickly repeating processes of their oxidative or flame reheating, could be $\sim 750-950^{\circ} \mathrm{C}$ (for most typical red or orange lightning balls), and so these nanoparticles could thermally emit a pulsating red or orange light with a ripple frequency high enough so that the light from ball lightning did not flicker.

Probably, however, total luminous radiation of nanoparticles-nanobatteries within ball lightning could arise from a combination of all three main light emitting processes: (1) nanoparticles-nanobatteries can emit faint light (faint thermal radiation) due to their direct oxidative heating or due to their periodic heating caused by a flame from combustion of combustible gases evolved in water vapour induced oxidative reactions; (2) nanoparticles-nanobatteries can emit pulsating luminous and ultra-violet radiation (as well as powerful pulsating wideband radio/micro waves) at moments of their partially or totally synchronized short circuits; (3) nanoparticles-nanobatteries can probably sometimes contain (or produce during oxidation) fluorescent or phosphorescent materials (e.g., some mixed metal oxides or sulphides), consequently, these particles-batteries can generate relatively low-temperature ultra-violet induced photoluminescence, also electroluminescence or cathodoluminescence.

Equally, many semi-conductor-based nanoparticles-nanobatteries can probably possess properties of quantum dots. In particular, the highly charged silicon based nanoparticles-silicon/air core-shell nanobatteries [20], continuously retransformed from the high-temperature, thermally dehydrated, $\mathrm{Si} / \mathrm{SiO}_{2}$ core/shell nanostructure into the lowtemperature, electrohydrated, $\mathrm{Si} / \mathrm{Si}(\mathrm{OH})_{4}$ core/shell nanostructure, can possess properties of quantum dots [31]. Such semi-conductor-based nanoparticles-nanobatteries, possessing properties of quantum dots, could be able to an intense low-temperature photo-, cathodo- and electroluminescence stimulated either by ultra-violet radiation of air-hydrogen flame or by collective discharge electron emission processes within the short-circuited nanobatteries.

Thus, a time-average temperature of many lightning balls can be relatively low, and consequently a light intensity from the ball lightning can practically be not connected with the time-average temperature of ball lightning.

\section{Steel Containing Objects and Aluminium Containing Objects Were Frequently Involved in the Directly Observed Processes of Ball Lightning Formation}

The involvement of iron/carbon-, and/or aluminium-based objects in ball lightning formation processes was repeatedly mentioned in numerous witnesses' reports $[8,9]$.

In particular, a lot of these reports were devoted to descriptions of a "high-temperature" ball lightning formation, in which a high-voltage arc evaporation of iron/carbonbased materials and further condensation of the evaporated materials in the form of a small smoke cloud could be the most probable events. At the same time, alternative, relatively "low-temperature" processes of ball lightning formation, that is those without the involvement of a visible electric arc, also were repeatedly described as connected with the frequent participation of the cast iron-based or steel based objects $[8,9]$.

Probably, it would be reasonable to assume that the unipolarly charged, iron-, and/or carbon-, and/or aluminium-based combustible aerosol particles could be initially generated from the electrode materials in all such ball lightning formation phenomena.

Therefore special attention to the process of water vapour induced oxidation of the electrostatically charged iron-, or carbon-, or aluminium-based aerosol nanoparticles, or their compositions in the form of electrostatically hydrated 
aggregated nanobatteries (or additionally in the form of electrostatically hydrated aggregated nanothermites/nanopyrotechnics) will be pertinent to our further discussion.

\section{Some Quantitative Estimations of a Charge-Dipole Interaction between a Charged Aerosol Nanoparticle and Surrounding Polar Gas Molecules}

It seems that on the one hand, a charge-dipole interaction between a charged aerosol nanoparticle and polar gas molecules from ambient air, for example, surrounding molecules of water vapour, can increase the number of collisions of these polar gas molecules with the charged nanoparticle, without a similar influence on the number of collisions of this particle with non-polar gas molecules.

On the other hand, it would be reasonable assume that the charge-dipole interaction between the charged aerosol nanoparticle and the surrounding polar gas molecules can in addition to increase kinetic energy of the polar molecules, accelerating these gas molecules towards the charged nanoparticle at a short distance of their mean free path from the charged nanoparticle.

Let us consider a simple case of oxidation of a combustible aerosol nanoparticle charged with the minimum possible charge, that is, charged with either a positive or negative elementary charge, $Q=|e|=1.6 \cdot 10^{-19}(\mathrm{C})$.

Again, with the purpose of simplification, let us assume that:

(a) this charged aerosol nanoparticle is spherical and it can freely and irregularly revolve on its axis due to continuous stochastic Brownian collisions with surrounding gas molecules;

(b) a time-average density of surplus electrostatic charge on the surface of this nanoparticle is practically equivalent to the time-average charge density on the surface of the same charged nanoparticle whose surplus electrostatic charge is localized in its centre, that is, the surplus electrostatic charge is quasidistributed on the surface of the nanoparticle, with the possibility of free fast migration of this charge on the nanoparticle surface.

In humid air, the majority of possible oxidative reactions on the surface of the discussed combustible nanoparticle can be caused by molecules of the two main competing atmospheric oxidants, oxygen gas and water vapour.

A flux of oxygen molecules incident upon the surface of the charged combustible nanoparticle suspended in humid air determines the frequency of collisions of the oxygen gas molecules with the surface of the charged nanoparticle, $f_{O}(\mathrm{~mol} / \mathrm{s})$. Correspondingly, this molecular flux determines also the rate of oxidative surface reactions caused by a fraction of the high-energy oxygen molecules possessing enough kinetic energy to climb the activation energy barriers of such oxygen induced oxidative surface reactions.

Similarly, a flux of water vapour molecules incident upon the surface of the charged combustible nanoparticle suspended in humid air determines the frequency of collisions of the water vapour molecules with the surface of the charged nanoparticle, $f_{W}(\mathrm{~mol} / \mathrm{s})$. And correspondingly, this molecular flux also determines the rate of oxidative surface reactions caused by a fraction of the high-energy water vapour molecules possessing enough kinetic energy to climb the activation energy barriers of such water vapour induced oxidative surface reactions.

\section{Humid Air Oxidation of a Spherical ( $\sim 2 \mathrm{~nm}$ in Diameter) Iron Metal Aerosol Nanoparticle Charged with the Minimum Positive Charge $Q=|e|=1.6 \cdot 10^{-19}(\mathbf{C})$ at a Relatively Low Temperature of about $300 \mathrm{~K}$.}

As a first example, let us consider a relatively lowtemperature process of humid air oxidation of a spherical $(\sim 2 \mathrm{~nm}$ in diameter) iron metal-based aerosol nanoparticle charged with the minimum possible positive charge $Q=$ $|e|=1.6 \cdot 10^{-19}(\mathrm{C})$, that is, with a single lost electron.

Let us assume that exothermic oxidation of this iron metal nanoparticle in the humid air is slow enough, because the water vapour and oxygen gas-induced intense growth of the passivating layers consisting of the mixed hydrated iron hydroxides, iron oxy-hydroxides, and iron oxides takes place on the surface of the iron metal nanoparticle during its humid air oxidation.

Assuming that the process of the mixed, water vapour and oxygen gas-induced exothermic oxidation of the discussed charged nanoparticle is slow enough, we could also suppose, for the first example, that both a time-average temperature of the nanoparticle surface, $T_{s}$, and a timeaverage temperature of ambient humid air around this nanoparticle, $T$, can remain approximately constant, and both these temperatures are approximately equal to the initial temperature of the nanoparticle oxidation process, that is, to $\sim 300 \mathrm{~K}$.

$$
T_{s}=T=300(\mathrm{~K}) .
$$

First, let us compare the flux of oxygen molecules with the competing flux of water vapour molecules incident upon the surface of the discussed charged nanoparticle in the humid air.

As oxygen molecules are non-polar and their permanent electric dipole moment, $p_{O}$, is zero, a flux of oxygen molecules incident upon the surface of an uncharged aerosol nanoparticle is practically equal to a flux of oxygen molecules incident upon the surface of the same charged nanoparticle. In other words, the frequency of collisions of non-polar oxygen molecules with the surface of an uncharged nanoparticle, $f_{O \text { unch }}(\mathrm{mol} / \mathrm{s})$, is practically equal to the frequency of collisions of the oxygen molecules with the surface of a similar but charged aerosol nanoparticle, $f_{O}(\mathrm{~mol} / \mathrm{s})$, which could be written as follows:

$$
f_{O \text { unch }}=f_{O}=A n_{O} v \sigma_{O}=A n_{O} v \pi R^{2},
$$

where $A$-a numerical factor, $n_{O}=0.21$-the mole fraction of oxygen molecules in humid air $(\mathrm{mol} / \mathrm{mol}), v$ - the mean 
speed of gas molecules at a given temperature $(\mathrm{m} / \mathrm{s}), \sigma_{O}=$ $\pi R^{2}$ - the cross section for collisions of surrounding nonpolar oxygen molecules with the charged (or also uncharged) aerosol nanoparticle $\left(\mathrm{m}^{2}\right), R$ - the radius of the nanoparticle (m).

If a combustible aerosol nanoparticle suspended in humid air is uncharged, non-polar molecules of atmospheric oxygen have a significant quantitative advantage over polar molecules of atmospheric water vapour when colliding (and also when reacting) with this nanoparticle, because the mole fraction of oxygen gas molecules is approximately tenfold greater than the mole fraction of water vapour molecules in humid air. Therefore, non-polar oxygen molecules can be a principal gas oxidant of uncharged combustible nanoparticles in humid air, while relatively few polar molecules of water vapour probably play a secondary role in the processes of humid air oxidation of uncharged nanoparticles (Figure 7).

The high-energy oxygen molecules possessing enough kinetic energy to climb the activation energy barriers of the oxidative surface reactions also can collide and react with the surface of uncharged combustible nanoparticle suspended in the humid air much more often in comparison with the relatively few high-energy water vapour molecules.

The preferential formation of the mixed, iron oxide layers on the surface of an uncharged iron nanoparticle could be a natural outcome of such a significant quantitative advantage of oxygen gas molecules over water vapour molecules in humid air. Indeed, it seems that oxygen induced formation of layers consisting predominantly of mixed iron oxides on the surface of the uncharged iron nanoparticle in humid air is a much more probable process than an alternative process of water vapour-induced formation of mixed hydrated, iron hydroxide, and/or iron oxy-hydroxide, surface layers.

However, it would also be reasonable to suppose that intense electrostatic hydration that locally converts iron oxides into iron hydroxides and iron oxy-hydroxides in humid air can still take place on oxidatively charged sites of the iron oxide surface of the originally uncharged iron nanoparticle, because electron migration from the iron metal core into the iron oxide $\mathrm{Fe}_{3} \mathrm{O}_{4} / \mathrm{Fe}_{2} \mathrm{O}_{3}$ semiconductor shell will continuously generate new negative surface charges even during a predominantly oxygen gas-induced oxidation of this originally uncharged iron nanoparticle.

At the same time, it seems that a different situation can arise when the discussed minimally charged iron nanoparticle is exposed to oxidation in humid air. Because water vapour molecules are high-polar, in contrast to nonpolar oxygen molecules, and the permanent electric dipole moment of a water vapour molecule is

$$
p_{W}=1.84(\mathrm{D})=0.6 \cdot 10^{-29}(\mathrm{C} \cdot \mathrm{m}),
$$

the intense charge-dipole attraction between the charged nanoparticle and surrounding polar molecules of water vapour can make an important contribution to the process of intense electrostatic nanoparticle hydration and, correspondingly, to a predominantly water vapour induced oxidation of the nanoparticle. Owing to the substantial charge-dipole attraction between the charged nanoparticle and surrounding polar molecules of water vapour, both the frequency and the intensity of collisions of the water vapour molecules with the surface of the charged aerosol nanoparticle can considerably exceed the corresponding frequency and intensity of collisions of these molecules with the surface of a similar uncharged nanoparticle (Figure 8).

The frequency of collisions of polar molecules of water vapour with a surface of the charged aerosol nanoparticle, $f_{W}$, can be represented as follows:

$$
f_{W}=A n_{W} v \sigma_{W},
$$

where $A$-a numerical factor, $n_{W} \sim 0.02$-the mole fraction of water vapour molecules in the humid atmosphere $(\mathrm{mol} / \mathrm{mol}), \quad v$-the mean speed of gas molecules at a given temperature $(\mathrm{m} / \mathrm{s}), \sigma_{W}$ - an effective cross section for collisions of surrounding polar molecules of water vapour with the charged aerosol nanoparticle $\left(\mathrm{m}^{2}\right)$.

The collision cross section $\sigma_{W}=\pi r^{* 2}$ can be determined estimating the value of potential energy of the charge-dipole interaction between the charged aerosol nanoparticle and polar molecule of water vapour from a gas microenvironment of the aerosol nanoparticle (taking into account that the size of the molecular dipole, that is, polar molecule of water vapour, is only about $0.2 \mathrm{~nm}$ )

$$
U(r)=-\frac{1}{4 \pi \varepsilon_{0}} \frac{p_{Q}}{r^{2}},
$$

where $1 / 4 \pi \varepsilon_{0}=9 \cdot 10^{9}(\mathrm{~m} / \mathrm{F}), p=p_{W}=0.6 \cdot 10^{-29}$-the permanent electric dipole moment of water vapour molecule $(\mathrm{C} \cdot \mathrm{m}), \mathbf{Q}=1.6 \cdot 10^{-19}$ - the charge of the nanoparticle $(\mathrm{C})$, $r$-the distance between the centre of the charged aerosol nanoparticle and the given molecule of water vapour from a gas microenvironment of the nanoparticle, $(\mathrm{m})$.

An electrostatic capture of the surrounding polar molecules of water vapour with a mean kinetic energy, $W$ $3 / 2 k T$, by the aerosol nanoparticle charged with a charge, $Q$, can take place at an effective distance- the effective capture radius $-r^{*}$, such that

$$
\frac{3}{2} k T=\frac{1}{4 \pi \varepsilon_{0}} \frac{p_{Q}}{r^{* 2}},
$$

where $k \approx 1.38 \cdot 10^{-23}$-the Boltzmann constant $(\mathrm{J} / \mathrm{K})$,

$T$ - the temperature of ambient humid air around the nanoparticle $(\mathrm{K})$.

As we assume that the temperature around the nanoparticle remains about $300 \mathrm{~K}$, the mean kinetic energy of the air molecules is about

$$
W=1.5 \cdot 1.38 \cdot 10^{-23}(\mathrm{~J} / \mathrm{K}) \cdot 300(\mathrm{~K})=6.21 \cdot 10^{-21}(\mathrm{~J}) .
$$

Correspondingly, a spherical volume of the radius of $r^{*}$ around the iron metal aerosol nanoparticle with an initial diameter of $2 \mathrm{~nm}$, suspended in humid air and charged with a minimum positive charge $Q=|e|=1.6 \cdot 10^{-19}(\mathrm{C})$, is an area where an effective electrostatic, charge-dipole capture of the surrounding polar water vapour molecules takes place at 


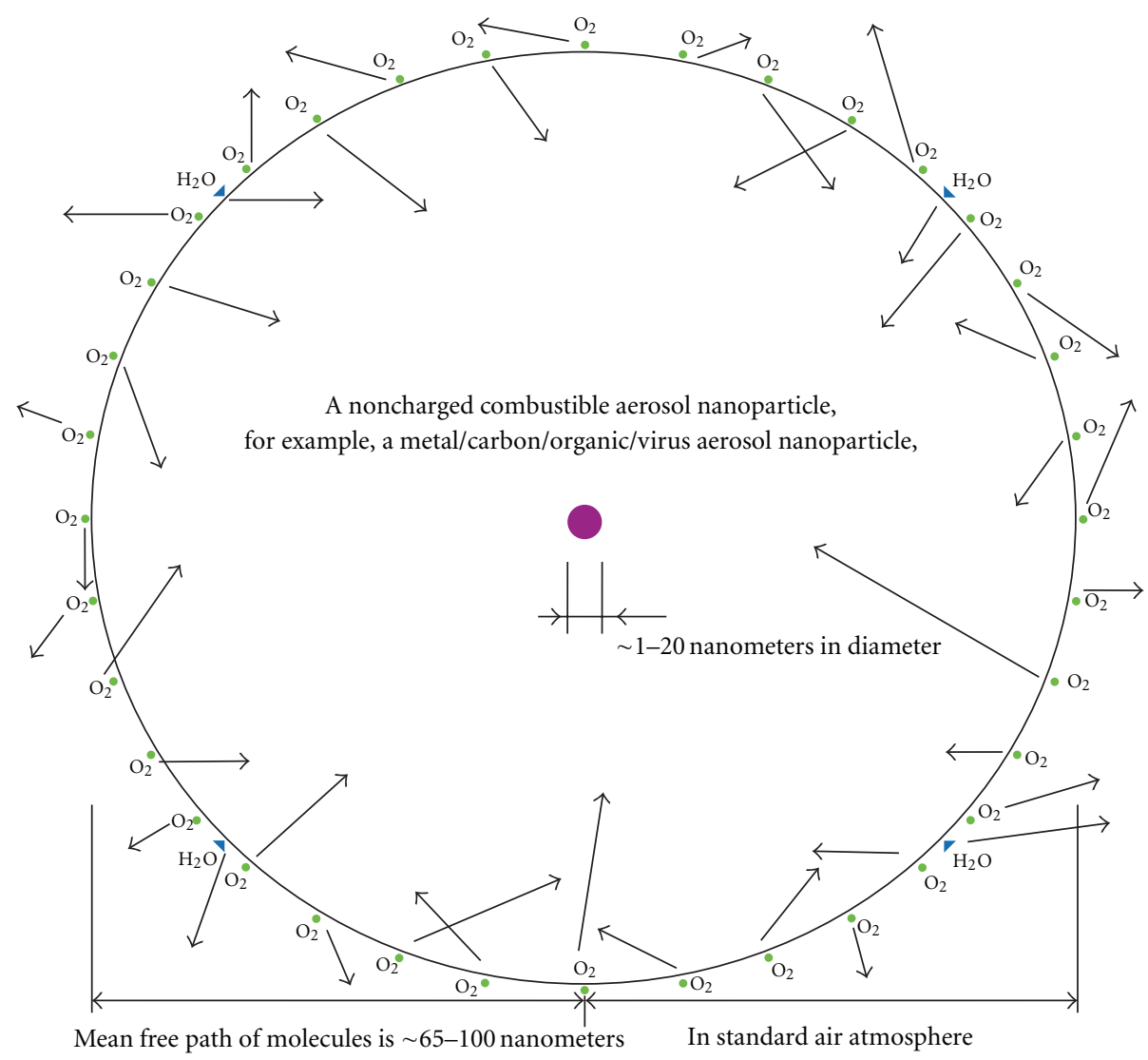

FIGURE 7: Even in a high humid air atmosphere, a high concentration of oxygen gas molecules creates the prerequisites for a preferential oxidation of uncharged combustible aerosol nanoparticles by oxygen molecules but not water vapour molecules. Water vapour, being another major air oxidant, with its much lower air concentration usually plays a secondary role in oxidative reactions on the surface of uncharged combustible nanoparticles. However, this situation can completely change during humid air oxidation of electrostatically charged nanoparticles.

the temperature of about $300 \mathrm{~K}$ and at a mean atmospheric pressure at sea level, $P$, equal to $101,325(\mathrm{~Pa})$.

As one can see the value of the effective radius of the electrostatic capture of the surrounding water vapour molecules by the minimally charged iron nanoparticle

$$
\begin{aligned}
r^{*} & =\sqrt{\frac{p Q}{4 \pi \varepsilon_{0} 3 / 2 k T}} \\
& =\sqrt{9 \cdot 10^{9} \frac{0,6 \cdot 10^{-29} \cdot 1,6 \cdot 10^{-19}}{3 / 2 \cdot 4,1 \cdot 10^{-21}}}=1.19 \cdot 10^{-9}(\mathrm{~m}),
\end{aligned}
$$

only slightly exceeds the $1 \mathrm{~nm}$ radius of the discussed charged nanoparticle and also it is much less than the mean free path of the ambient gas molecules, $l \approx 10^{-7}(\mathrm{~m})$, under the given conditions of temperature and pressure.

The corresponding cross section for collisions of the surrounding polar molecules of water vapour with the discussed charged iron nanoparticle (and probably also for electrostatic capture of these polar molecules by the charged iron nanoparticle) is

$$
\sigma_{W} \sim \pi r^{* 2}=4.5 \cdot 10^{-18}\left(\mathrm{~m}^{2}\right) .
$$

This cross section also only slightly (approximately one and a half times) exceeds a "geometrical" cross-section for possible collisions of this charged nanoparticle with the surrounding non-polar oxygen molecules:

$$
\sigma_{O} \sim 3.14 \cdot 10^{-18}\left(\mathrm{~m}^{2}\right) .
$$

Indeed as one can see the cross-section for collisions of the surrounding polar molecules of water vapour with a minimally charged $2 \mathrm{~nm}$ diameter aerosol nanoparticle can only slightly exceed the cross-section for collisions of this aerosol nanoparticle with the surrounding non-polar molecules of oxygen gas due to a contribution of the charge-dipole attraction between such a charged aerosol nanoparticle and surrounding polar molecules of water vapour.

However, the situation strongly changes when the diameter of the minimally charged nanoparticle becomes less 


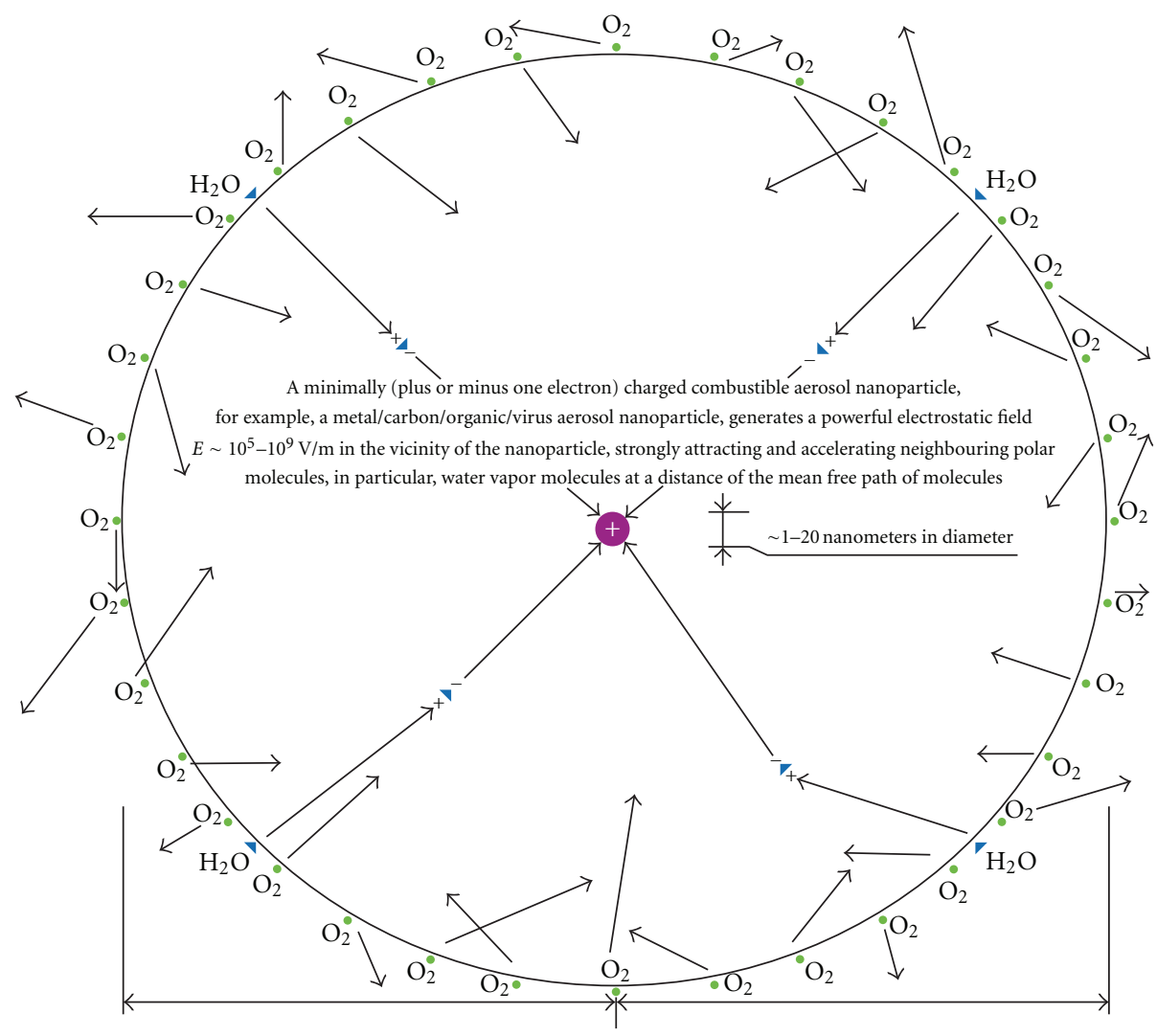

Mean free path of molecules is 65-100 nanometers

In standard air atmosphere

FIGURE 8: Powerful charge-dipole attraction between charged aerosol nanoparticles and the surrounding molecules of water vapour in humid air bends the trajectories of the polar molecules and also additionally accelerates these polar molecules in the direction of the charged nanoparticles at a distance of the air molecules' mean free path. On the other hand, nonpolar air molecules, such as oxygen gas molecules (or nitrogen gas, or carbonic gas) are practically not subject to the influence of charge-dipole attraction in the vicinity of charged aerosol nanoparticles, and so the absolute values of kinetic energy and the directions of movement of the nonpolar air molecules around the charged nanoparticles are practically not changed by forces of the charge-dipole attraction. A significant electrostatic acceleration of water vapour molecules in the vicinity of the charged combustible aerosol nanoparticles substantially increases reactivity of these molecules. Thus, the oxidizing efficiency of the polar molecules of water vapour in relation to the charged combustible nanoparticles considerably exceeds the alternative oxidizing efficiency of nonpolar, correspondingly electrostatically nonaccelerated molecules of oxygen gas, though approximately a tenfold excess of oxygen gas molecules' concentration, in comparison with the concentration of water vapour molecules, normally takes place in air atmosphere.

than $1 \mathrm{~nm}$. For example, it is easy to estimate that a cross section for collisions of a minimally charged $1 \mathrm{~nm}$ diameter aerosol nanoparticle with the surrounding water vapour molecules can approximately by sixfold exceed the cross section for collisions of a similar minimally charged aerosol nanoparticle with the surrounding non-polar gas molecules, including oxygen gas molecules.

Correspondingly, in a humid atmosphere, electrostatic hydration of such small charged aerosol nanoparticles (or such small substrate-integrated charged nanoscale surface sites) can be much more intense than alternative hydration of similar uncharged nanoparticles (or the same uncharged surface sites of substrate-integrated nanostructures).

In fact, spontaneously or artificially minimally charged aerosol nanoparticles or substrate-integrated nanostructures with characteristic sizes of $\leq 1 \mathrm{~nm}$ are extremely widespread objects, both the nanotechnological (e.g., nanotube ends, fullerenes, etc.) and, particularly, biological origin (e.g., peripheral and transmembrane cell receptors).

In a water vapour-containing atmosphere, electrostatical hydration of charged aerosol nanoparticles (or charged substrate-integrated nanostructures) can be particularly intense within a small charged area with a radius of $\leq 1 \mathrm{~nm}$, where the local charge (e.g., an adsorbed hydrated ion) is located at the centre of this area that is most actively bombarded by the surrounding polar molecules, locally electrostatically accelerated. Because this zone with a highest charge density and with a diameter of $\leq 2 \mathrm{~nm}$, exposed to the most active attack by surrounding polar molecules, is able to migrate (together with its charge) onto the surface of an individual combustible aerosol particle or along chains consisting of aggregated aerosol particles, then the processes 
of charge-catalyzed water vapour-induced oxidation can involve a high number of initially uncharged particles.

However, let us return to the question about humid air oxidation of the discussed minimally charged spherical iron nanoparticle with a diameter of $\sim 2 \mathrm{~nm}$.

Despite the fact that $\sigma_{W} \approx 1.5 \sigma_{O}$, when comparing oxygen and water vapour molecular fluxes incident upon the surface of the discussed iron nanoparticle, one can see that the frequency of collisions of the surrounding polar molecules of water vapour with the surface of this nanoparticle, $f_{W}=A n_{W} v \sigma_{W}$, is still much lower than the frequency, $f_{O}=A n_{O} v \sigma_{O}$, of alternative collisions of the surrounding oxygen molecules with this surface

$$
f_{W}<f_{O}
$$

because the mole fraction of atmospheric molecules of oxygen gas $n_{O}=0.21$ is much higher than the mole fraction of water vapour molecules $n_{W} \sim 0.02$ in humid air.

It is clear that this result does not depend on whether the discussed combustible nanoparticle is charged positively, with a single lost electron, or if it is charged negatively, with a single surplus electron.

Thus, we can draw a conclusion that in humid air the charge-dipole attraction between the minimally charged aerosol nanoparticle with a diameter $2 \mathrm{~nm}$ or greater and the surrounding polar molecules of water vapour does not provide a noticeable quantitative advantage of polar molecules of water vapour over non-polar molecules of oxygen in access to a surface of this minimally charged nanoparticle.

However, there is another important aspect to be discussed, namely, the influence of the charge-dipole attraction between the charged aerosol nanoparticle and the surrounding polar molecules of water vapour on the intensity of collisions of these polar molecules with the surface of the nanoparticle.

Indeed, in humid air the charge-dipole attraction between the discussed charged combustible nanoparticle and the surrounding polar molecules of water vapour are not able to appreciably increase the frequency of collisions of the polar molecules with such a large minimally charged nanoparticle.

But the charge-dipole attraction in addition can substantially accelerate the surrounding polar molecules of water vapour in the direction of the nanoparticle at the distance of the mean free path of ambient gas molecules $l \approx 10^{-7}(\mathrm{~m})$ under the given conditions of temperature and pressure, increasing the number of high-energy molecules of water vapour with enough kinetic energy to climb the activation energy barriers of the oxidative reactions induced by water vapour on the surface of the charged combustible aerosol nanoparticle.

Let us now estimate this possibility in order to compare the rates of the corresponding oxidative reactions potentially induced on the surface of the charged iron nanoparticle by either non-polar high-energy molecules of oxygen or electrostatically extra accelerated polar high-energy molecules of water vapour.

\section{Additional Average kinetic Energy Acquired by Polar Molecules of Water Vapour in an Electrostatic Field of a Minimally Charged Aerosol Nanoparticle at the Mean Free Path Distance from the Surface of This Nanoparticle}

Additional average kinetic energy $\varepsilon_{1}$ acquired by the polar molecule of water vapour in the electrostatic field of the iron nanoparticle with an initial diameter of $2 \mathrm{~nm}$, suspended in humid air and charged with the minimum positive charge $Q=|e|=1.6 \cdot 10^{-19}(\mathrm{C})$, at an acceleration distance of the mean free path from the nanoparticle is

$$
\varepsilon_{1}=\frac{1}{4 \pi \varepsilon_{0}} \frac{p Q}{R^{2}}-\frac{1}{4 \pi \varepsilon_{0}} \frac{p Q}{l^{2}} \approx \frac{1}{4 \pi \varepsilon_{0}} \frac{p Q}{R^{2}},
$$

where $1 /\left(4 \pi \varepsilon_{0}\right)=9 \cdot 10^{9}(\mathrm{~m} / \mathrm{F}), p=p_{W}=0.6$. $10^{-29}$ - the permanent electric dipole moment of a water vapour molecule $(\mathrm{C} \cdot \mathrm{m}), Q=1.6 \cdot 10^{-19}$-the charge of the nanoparticle $(\mathrm{C}), R=1.0 \cdot 10^{-9}$-the radius of the nanoparticle $(\mathrm{m}), l \approx 1.0 \cdot 10^{-7}$ - the mean free path of ambient gas molecules $(\mathrm{m})$.

Thus, the additional average kinetic energy $\varepsilon_{1}$ acquired by the polar molecule of water vapour in the electrostatic field of the discussed minimally charged nanoparticle at a distance of the mean free path from the nanoparticle is

$$
\varepsilon_{1}=9 \cdot 10^{9} \cdot 0.6 \cdot 10^{-29} \cdot 1.6 \cdot \frac{10^{-19}}{10^{-18}} \approx 8.6 \cdot 10^{-21}(\mathrm{~J}) .
$$

The additional average energy of electrostatic acceleration of polar molecules of water vapour appreciably, more than twice (!), exceeds the characteristic thermal energy, $k T$, of the surrounding air molecules at a temperature of about $300 \mathrm{~K}$.

$$
k T=4.1 \cdot 10^{-21}(\mathrm{~J}) .
$$

According to [21], "the activation energy for the reaction of Fe with $\mathrm{O}_{2}$ was determined to be $32 \pm 6 \mathrm{~kJ} / \mathrm{mol}$ for exposure times of $20-2000 \mathrm{~s}$ and $28 \pm 3 \mathrm{~kJ} / \mathrm{mol}$ for the reaction of $\mathrm{Fe}$ with water vapour for exposure times of 100-2000s".

Correspondingly, we will suppose that the activation energy for the process of water vapour induced oxidation of iron metal nanoparticle, $E_{a W}(\mathrm{~J} / \mathrm{mol})$, also is about 28,000 $(\mathrm{J} / \mathrm{mol})$ at a temperature of about $300 \mathrm{~K}$. And correspondingly, the activation energy for an alternative competing reaction of oxygen gas induced oxidation of iron metal nanoparticle, $E_{a O}(\mathrm{~J} / \mathrm{mol})$, also is about $32,000(\mathrm{~J} / \mathrm{mol})$ at the given temperature.

These, practically equivalent activation energies, $E_{a W} \approx$ $E_{a O}=E_{a} \approx 30,000(\mathrm{~J} / \mathrm{mol})$, can be calculated per one molecule, $\varepsilon_{a}(\mathrm{~J})$, and, correspondingly, are about

$$
\varepsilon_{a}=\frac{E_{a}}{N_{A}} \approx \frac{30,000}{\left(6.02 \cdot 10^{23}\right)} \approx 5 \cdot 10^{-20}(\mathrm{~J}),
$$

where $N_{A} \approx 6.02 \cdot 10^{23}$ - the Avogadro constant $\left(\mathrm{mol}^{-1}\right)$.

One can see that the ratio of the activation energy, $\varepsilon_{a}(\mathrm{~J})$, for both reactions of iron oxidation (with the participation 
of either the surrounding water vapour or oxygen gas molecules) to the characteristic thermal energy, $k T$, of these air molecules at the temperature of about $300 \mathrm{~K}$ is

$$
\frac{\varepsilon_{a}}{k T}=\frac{5 \cdot 10^{-20}}{4.1 \cdot 10^{-21}}=12,2 .
$$

Only high energy molecules of water vapour and oxygen gas, with kinetic energy $\varepsilon \geq \varepsilon_{a}$, can react with the discussed minimally charged aerosol iron nanoparticle.

The number of such reactive molecules is equal to

$$
\begin{aligned}
\Delta N & =N \frac{2}{\sqrt{\pi}} \frac{1}{(k T)^{3 / 2}} \int_{\varepsilon_{a}}^{\infty} \sqrt{\varepsilon} e^{-\varepsilon /(k T)} d \varepsilon \\
& =N \frac{2}{\sqrt{\pi}} \int_{\varepsilon_{a} /(k T)}^{\infty} \sqrt{\varepsilon^{\prime}} e^{-\varepsilon^{\prime}} d \varepsilon^{\prime}
\end{aligned}
$$

By integration by parts and a series expansion, we can approximately calculate a relative quantity of reactive oxygen gas molecules possessing enough kinetic energy to climb the activation energy barrier of the oxygen-induced oxidative reactions on the surface of the discussed charged iron nanoparticle at a temperature of about $300 \mathrm{~K}$

$$
\begin{aligned}
\frac{\Delta N_{O}}{N_{O}} & =\frac{2}{\sqrt{\pi}} \int_{12.2}^{\infty} \sqrt{\varepsilon^{\prime}} e^{-\varepsilon^{\prime}} d \varepsilon^{\prime} \\
& \approx \frac{2}{\sqrt{\pi}} e^{-12.2}\left(\sqrt{12.2}+\frac{1}{2 \sqrt{12.2}}\right)=2.1 \cdot 10^{-5} .
\end{aligned}
$$

Clearly, to calculate a relative quantity (a fraction) of reactive water vapour molecules possessing enough kinetic energy to climb the activation energy barrier of the water vapour induced oxidative reactions on the surface of the discussed charged iron nanoparticle at the temperature of about $300 \mathrm{~K}$, it is necessary to integrate the above expression with the lower limit of integration

$$
\frac{\varepsilon_{a}}{\left(k T+\varepsilon_{1}\right)}=\frac{5 \cdot 10^{-20}}{\left(4.1 \cdot 10^{-21}+8.6 \cdot 10^{-21}\right)}=3.9 .
$$

Let us recall that $\varepsilon_{1} \approx 8.6 \cdot 10^{-21}(\mathrm{~J})$ is the additional average kinetic energy acquired by the polar molecule of water vapour in the electrostatic field of minimally charged nanoparticle at a distance of the mean free path from the nanoparticle.

Correspondingly, the fraction of the reactive water vapour molecules possessing enough kinetic energy to climb the activation energy barrier of the water vapour-induced oxidative reactions on the surface of the discussed charged iron nanoparticle at a temperature of about $300 \mathrm{~K}$ is

$$
\begin{aligned}
\frac{\Delta N_{H}}{N_{H}} & =\frac{2}{\sqrt{\pi}} \int_{3.9}^{\infty} \sqrt{\varepsilon^{\prime}} e^{-\varepsilon^{\prime}} d \varepsilon^{\prime} \\
& \approx \frac{2}{\sqrt{\pi}} e^{-3.9}\left(\sqrt{3.9}+\frac{1}{2 \sqrt{3.9}}\right)=0.049 .
\end{aligned}
$$

As one can see, this fraction of the additionally electrostatically accelerated reactive water vapour molecules, $\Delta N_{H} / N_{H}=4.9 \cdot 10^{-2}$, substantially exceeds the alternative fraction of the reactive oxygen gas molecules $\Delta N_{O} / N_{O}=$ $2.1 \cdot 10^{-5}$.

Recalculating these relative quantities of oxygen and water vapour reactive molecules, which are able to climb the activation energy barriers of the oxidative reactions on the surface of the discussed charged iron nanoparticle at a temperature of about $300 \mathrm{~K}$, per one mole of humid air, and taking into account the concentration difference (typical for humid air) between these main air oxidants, we have

$$
N_{O}=N_{A} \cdot n_{O}=6.02 \cdot 10^{23} \cdot 0.21=1.26 \cdot 10^{23},
$$

where $N_{O}$ - the quantity of oxygen molecules in one mole of humid air, $N_{A} \approx 6.02 \cdot 10^{23}$ — the Avogadro constant $\left(\mathrm{mol}^{-1}\right)$, $n_{O}=0.21$ - the mole fraction of oxygen molecules in humid air $(\mathrm{mol} / \mathrm{mol})$.

Analogously,

$$
N_{W}=N_{A} \cdot n_{W}=6.02 \cdot 10^{23} \cdot 0.02=1.2 \cdot 10^{22} .
$$

where $N_{W}$-the quantity of water vapour molecules in one mole of humid air $N_{A} \approx 6.02 \cdot 10^{23}$ - the Avogadro constant $\left(\mathrm{mol}^{-1}\right), n_{w} \sim 0.02$ - the mole fraction of water vapour molecules in humid air $(\mathrm{mol} / \mathrm{mol})$, which could for example correspond to normal summer atmospheric conditions with a relative humidity of $\sim 80 \%$ and a temperature of $\sim 300 \mathrm{~K}$ (i.e., $\sim 27^{\circ} \mathrm{C}$ ).

Now, we can calculate:

(a) the absolute quantity of reactive oxygen molecules, which are able to climb the activation energy barrier of the oxidative reactions on the surface of the discussed minimally charged iron nanoparticle at a temperature of about $300 \mathrm{~K}$, and which are contained in one mole of humid air:

$$
\begin{aligned}
\Delta N_{O} & =N_{O} \cdot 2.1 \cdot 10^{-5} \\
& =1.26 \cdot 10^{23} \cdot 2.1 \cdot 10^{-5}=2.6 \cdot 10^{18},
\end{aligned}
$$

(b) the absolute quantity of reactive molecules of water vapour, which are able to climb the activation energy barrier of oxidative reactions on the surface of the discussed minimally charged iron nanoparticle at a temperature of about $300 \mathrm{~K}$, and which are contained in one mole of humid air

$$
\Delta N_{H}=N_{H} \cdot 0.049=1.2 \cdot 10^{22} \cdot 0.049=588 \cdot 10^{18} .
$$

As one can see, the number of reactive water vapour molecules, which are able to oxidize the discussed minimally charged iron nanoparticle suspended in humid air at a temperature of $\sim 300 \mathrm{~K}$ is

$$
\frac{\Delta N_{H}}{\Delta N_{O}}=\frac{588 \cdot 10^{18}}{2.6 \cdot 10^{18}}=226,
$$

times larger than the number of reactive oxygen molecules, which are able to oxidize the charged surface of this 
nanoparticle under similar atmospheric conditions when the mole fraction of water vapour molecules, $n_{W}$, in humid air is $\sim 0.02$.

If the mole fraction of water vapour molecules, $n_{W}$, in humid air is only 0.01 (which could, e.g., correspond to atmospheric conditions with a relative humidity of $\sim 57 \%$ and an air temperature of $\sim 293 \mathrm{~K}$, i.e., $\sim 20^{\circ} \mathrm{C}$, or which could equally correspond to atmospheric conditions with a relative humidity of $\sim 100 \%$ and an air temperature of $\sim 282 \mathrm{~K}$, i.e., $\sim 9^{\circ} \mathrm{C}$ ), even in these cases the number of water vapour molecules, which are able to oxidize the discussed minimally charged aerosol iron nanoparticle is only

$$
\frac{\Delta N_{H}}{\Delta N_{O}}=\frac{226}{2}=113
$$

times larger than the number of oxygen molecules, which are able to oxidize this charged nanoparticle under such conditions.

One can see that despite the relatively small number of polar molecules of water vapour in normal humid air, they can become a major air oxidant of charged aerosol nanoparticles (or, analogously, charged surface nanostructures) due to the influence of the charge-dipole interaction between these charged aerosol nanoparticles (charged surface nanostructures) and surrounding polar molecules of water vapour.

Indeed, it is interesting that the above-calculated number of reactive molecules of water vapour, which are able to oxidize charged iron nanoparticles can hundreds of times exceed the number of reactive molecules of oxygen gas, competitively attacking these charged nanoparticles at an air temperature of about $300 \mathrm{~K}$ in normal conditions of widely varying air humidity. Although this effect of preferential oxidation of charged combustible nanoparticles by water vapour molecules rather than by oxygen gas molecules contained in humid air is shown for a possible process of oxidation of charged iron metal nanoparticles, it seems that the similar effect can take place during humid air oxidation of a lot of various, not only metal-based combustible particles.

The effect of electrostatic, charge-dipole acceleration of polar gas molecules in immediate proximity to a charged aerosol nanoparticle does not depend on the polarity of the nanoparticle charge. Thus, both the positively charged nanoparticles and negatively charged nanoparticles equally can electrostatically attract and accelerate the surrounding polar gas phase molecules, including water vapour molecules, at the distance of the mean free path of these molecules from the charged nanoparticles.

The effect of the electrostatic acceleration of polar gas molecules near to the charged nanoparticle also does not depend on the specific material components of the charged nanoparticle. During oxidation, a final diameter of the completely oxidized nanoparticle, originally consisting of iron metal, can be only slightly increased in comparison with the initial diameter of the iron metal nanoparticle due to the oxidative growth of the iron hydroxide and/or iron oxyhydroxide-based porous surface layers, which are less dense than iron metal. However, it is simple to estimate that a change in the diameter of the iron metal nanoparticle during this oxidation process does not exceed $50 \%$ of the initial diameter of the iron metal nanoparticle.

Now, let us consider the analogous process of humid air oxidation of the spherical iron metal aerosol nanoparticle with a diameter of $2 \mathrm{~nm}$ charged with the minimum positive charge $Q=|e|=1.6 \cdot 10^{-19}(\mathrm{C})$ at the higher temperature of about $573 \mathrm{~K}$.

\section{Humid Air Oxidation of a Spherical ( $\sim 2 \mathrm{~nm}$ in diameter) Iron Metal Aerosol Nanoparticle Charged With the Minimum Positive Charge $Q=|e|=1.6 \cdot 10^{-19}(\mathbf{C})$ at a Relatively High Temperature of about $573 \mathrm{~K}$}

Let us suppose again that in humid air the mole fraction of molecules of water vapour, $n_{W}$, is $\sim 0.02$. Let us suppose also that at a temperature of $\sim 573 \mathrm{~K}$ (i.e., $\sim 300^{\circ} \mathrm{C}$ ), for the competing processes of water vapour- and oxygen gas induced oxidation of iron metal surface, that is, for the oxidative processes with high-temperature parabolic kinetics, an average value, $E_{a}(\mathrm{~J} / \mathrm{mol})$, of both activation energies (i.e., the activation energy for water vapour-induced oxidation of iron nanoparticle, $E_{a W}(\mathrm{~J} / \mathrm{mol})$, and the activation energy for oxygen gas induced oxidation of this nanoparticle $E_{a O}$ $(\mathrm{J} / \mathrm{mol}))$ can be of $\sim 150,000(\mathrm{~J} / \mathrm{mol})[21,32,33]$

$$
E_{a} \approx E_{a W} \approx E_{a O} \sim 150,000(\mathrm{~J} / \mathrm{mol}) . s
$$

An essential deceleration of the oxidative processes on the surface of the iron metal nanoparticle takes place due to a passivating influence of the growing surface layers, which probably consist mainly of mixed iron hydroxide $\mathrm{Fe}(\mathrm{OH})_{2}$ and iron oxy-hydroxide $\mathrm{FeOOH}$ (at this temperature), and which are exposed to continuous saturation with hydrogen gas evolved on and within the growing surface layers [22] due to reaction between iron metal nanoparticle and electrostatically absorbed water vapour. Thus, the growing iron hydroxide-based surface layers and evolving hydrogen gas can probably significantly inhibit iron metal oxidation in humid air (Figure 9).

Thus, at $T \approx 573 \mathrm{~K}$,

$$
\begin{aligned}
& k T \approx 1.38 \cdot 10^{-23} \cdot 573=7.91 \cdot 10^{-21}(\mathrm{~J}), \\
& \varepsilon_{a}=\frac{E_{a}}{N_{A}} \approx \frac{150,000}{\left(6.02 \cdot 10^{23}\right)} \approx 2.5 \cdot 10^{-19}(\mathrm{~J}),
\end{aligned}
$$

where $N_{A} \approx 6.02 \cdot 10^{23}$ — the Avogadro constant $\left(\mathrm{mol}^{-1}\right)$.

Consequently, in the case of this relatively high temperature humid air oxidation of the charged iron metal aerosol nanoparticle

$$
\begin{gathered}
\frac{\varepsilon_{a}}{k T}=31.6 \\
\frac{\varepsilon_{a}}{\left(k T+\varepsilon_{1}\right)}=\frac{2.5 \cdot 10^{-19}}{\left(7.91 \cdot 10^{-21}+8.6 \cdot 10^{-21}\right)}
\end{gathered}=15.2 .
$$

Let us again recall that $\varepsilon_{1} \approx 8.6 \cdot 10^{-21}(\mathrm{~J})$ is the additional average kinetic energy acquired by the polar molecule of 


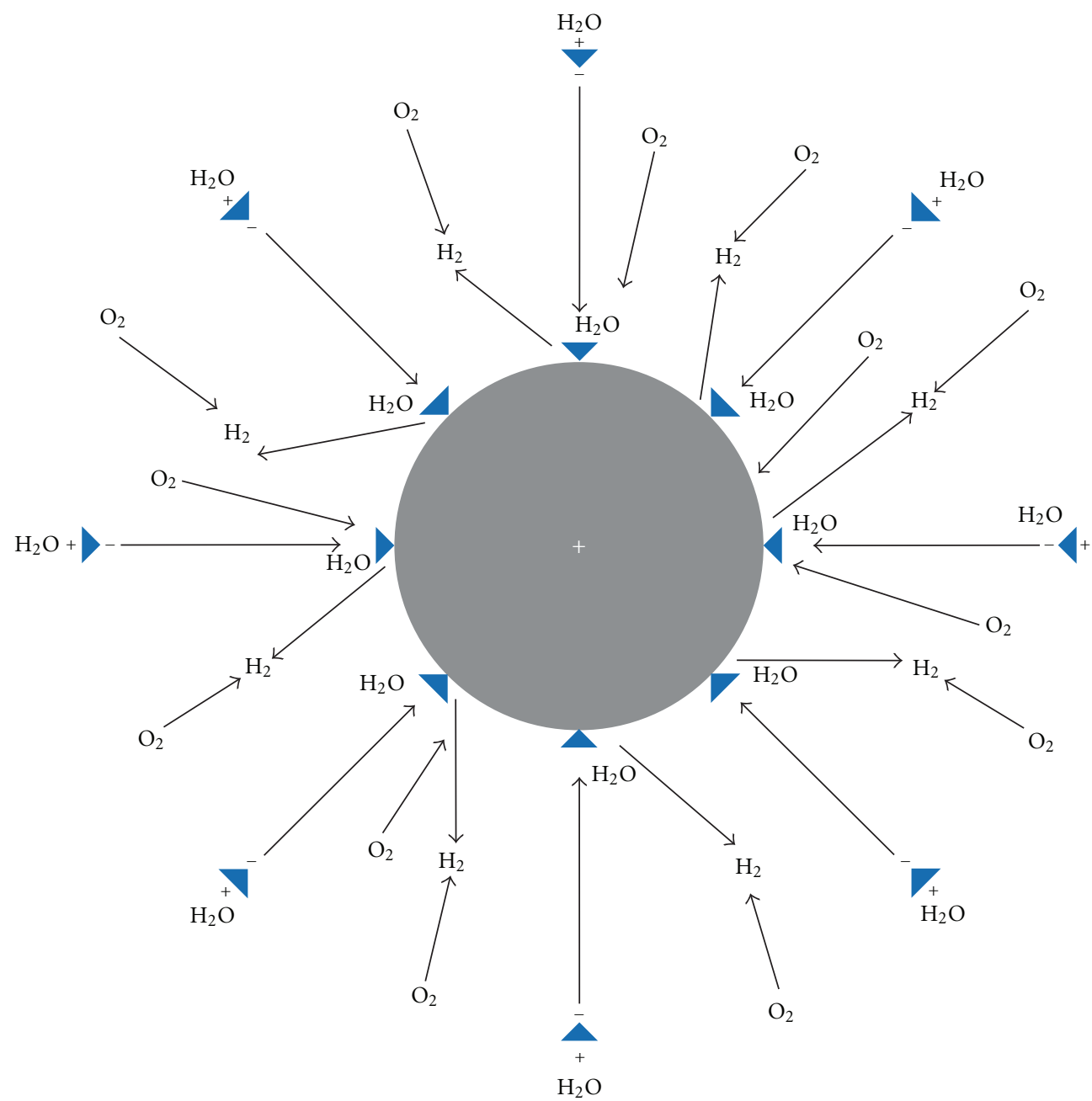

FigURE 9: An attack of surrounding molecules of water vapour against charged combustible aerosol nanoparticles is much more intense in comparison with an alternative attack of nonpolar molecules of oxygen gas, because a powerful charge-dipole acceleration of the polar molecules of water vapour towards the charged nanoparticles takes place in immediate proximity to the nanoparticles. In such cases, at an ambient air temperature of $\sim 300 \mathrm{~K}$ the only elementary charge, either a positive or negative one, when located on the surface of the nanoparticle, can significantly (approximately twice) increase the kinetic energy of the collisions of the surrounding water vapour molecules with the surface of this charged nanoparticle due to the powerful charge-dipole attraction of the water vapour molecules to the charged surface or, more precisely, due to a powerful electrostatic attraction of surrounding water vapour molecular dipoles into regions on the surface of the charged nanoparticle with a maximum charge density and a corresponding maximum local electrostatic intensity. Even at ambient air temperatures of $\sim 500-700 \mathrm{~K}$, the water vapour molecules, electrostatically accelerated around the highly charged aerosol nanoparticles, can collide with the surface of the nanoparticles with kinetic energy still greater than the average kinetic energy of the rest of the air molecules in a thermal equilibrium. This means that accelerated polar molecules of water vapour can effectively oxidize the charged combustible nanoparticles in humid air under such temperature conditions when thermally equilibrium nonpolar molecules, including oxygen gas molecules, remain practically inactive. In humid air, molecules of water vapour can become a major oxidant in relation to various highly charged combustible nanoparticles, in both their aerosol and/or precipitated form.

water vapour in the electrostatic field of minimally charged nanoparticle at a distance of the mean free path from the nanoparticle.

Thus, the fraction of the reactive oxygen gas molecules, that is, oxygen gas molecules possessing enough kinetic energy to climb the activation energy barrier of the oxygen gas-induced oxidative reactions on the surface of the discussed iron nanoparticle at a temperature of about $573 \mathrm{~K}$, is

$$
\begin{aligned}
\frac{\Delta N_{O}}{N_{O}} & =\frac{2}{\sqrt{\pi}} \int_{31.6}^{\infty} \sqrt{\mathcal{\varepsilon}^{\prime}} e^{-\varepsilon^{\prime}} d \varepsilon^{\prime} \\
& \approx \frac{2}{\sqrt{\pi}} e^{-31.6}\left(\sqrt{31.6}+\frac{1}{2 \sqrt{31.6}}\right)=1.22 \cdot 10^{-13} .
\end{aligned}
$$

Consequently, the competing fraction of the reactive water vapour molecules possessing enough kinetic energy to climb 
the activation energy barrier of the water vapour-induced oxidative reactions on the surface of the discussed minimally charged iron nanoparticle at a temperature of about $573 \mathrm{~K}$ is

$$
\begin{aligned}
\frac{\Delta N_{H}}{N_{H}} & =\frac{2}{\sqrt{\pi}} \int_{15.2}^{\infty} \sqrt{\varepsilon^{\prime}} e^{-\varepsilon^{\prime}} d \varepsilon^{\prime} \\
& \approx \frac{2}{\sqrt{\pi}} e^{-15.2}\left(\sqrt{15.2}+\frac{1}{2 \sqrt{15.2}}\right)=1.14 \cdot 10^{-6} .
\end{aligned}
$$

Thus, in this relatively high temperature case of oxidation of the charged iron metal particle:

(a) the absolute quantity of the reactive non-polar molecules of oxygen gas, which is able to climb the activation energy barrier of the oxidative reactions on the surface of the discussed minimally charged nanoparticle at a temperature of about $573 \mathrm{~K}$, and which are contained in one mole of humid air

$$
\begin{aligned}
\Delta N_{O} & =N_{O} \cdot 1.22 \cdot 10^{-13} \\
& =1.26 \cdot 10^{23} \cdot 1.22 \cdot 10^{-13}=1.53 \cdot 10^{10} .
\end{aligned}
$$

(b) the absolute quantity of the reactive polar molecules of water vapour, which is able to climb the activation energy barrier of the oxidative reactions on the surface of the discussed minimally charged nanoparticle at a temperature of about $573 \mathrm{~K}$, and which are contained in one mole of humid air

$$
\begin{aligned}
\Delta N_{H} & =N_{H} \cdot 1.14 \cdot 10^{-6} \\
& =1.2 \cdot 10^{22} \cdot 1.14 \cdot 10^{-6}=1.37 \cdot 10^{16} .
\end{aligned}
$$

As one can see, the number of reactive water vapour molecules, which are able to oxidize the discussed minimally charged iron nanoparticle suspended in humid air at a temperature of $\sim 573 \mathrm{~K}$ is

$$
\frac{\Delta N_{\mathrm{H}}}{\Delta N_{\mathrm{O}}}=\frac{1.37 \cdot 10^{16}}{1.53 \cdot 10^{10}}=0.9 \cdot 10^{6} .
$$

times larger than the number of reactive oxygen gas molecules, which are able to oxidize the charged surface of this nanoparticle under such relatively high-temperature conditions of humid air oxidation when the mole fraction of water vapour molecules, $n_{W}$, in humid air is $\sim 0.02$.

Indeed, an unexpectedly large (approximately one million times!) difference is found between the number of reactive water vapour molecules, which are able to oxidize the minimally charged iron nanoparticle in humid air and the number of alternative reactive molecules of oxygen gas, competitively participating in this humid air oxidation. This means that relatively few polar molecules of water vapour in humid air, being additionally electrostatically accelerated by their charge-dipole attraction towards the minimally charged iron nanoparticle, become practically the only air oxidant of the discussed minimally charged iron nanoparticle, and non-polar molecules of oxygen gas actually do not take part in humid air oxidation of this charged nanoparticle both at an oxidation temperature of $\sim 300 \mathrm{~K}$ and especially at an oxidation temperature of $\sim 573 \mathrm{~K}$. Thus, probably, that one of the main oxidative reactions on the surface of the minimally charged iron nanoparticle will be a water vapourinduced exothermal reaction of oxidation of iron metal with formation of the hydrated, iron hydroxide $\mathrm{Fe}(\mathrm{OH})_{2}$ and/or iron oxy-hydroxide $\mathrm{FeOOH}$-based porous electrolyte surface layers with synchronous evolution of hydrogen gas

$$
\begin{aligned}
& 3 \mathrm{Fe}_{(\text {nanoparticle })}+6 \mathrm{H}_{2} \mathrm{O}_{\text {(vapour) }} \\
& \quad=\mathrm{Fe}(\mathrm{OH})_{2}+2 \mathrm{FeOOH}+4 \mathrm{H}_{2} \uparrow .
\end{aligned}
$$

The solid reaction products, growing on the surface of the charged iron nanoparticle in the form of the hydrated electrolyte layers, will limit diffusion and direct oxidation of the nanoparticle by external neutral oxidizing species, but at the same time, these electrolyte layers will effectively transport:

(a) ionized oxidizing species, such as $\mathrm{OH}^{-}$ions and $\mathrm{O}_{2}{ }^{-}$ ions into the iron core,

(b) iron metal ions, such as $\mathrm{Fe}^{2+}$, or $\mathrm{Fe}^{3+}$, or $\mathrm{Fe}(\mathrm{OH})^{+}$, $\mathrm{Fe}(\mathrm{OH})^{2+}$, in the opposite direction, to the outer surface of the iron metal nanoparticle.

The solid reaction products in the form of the hydrated hydroxide or oxy-hydroxide surface electrolyte layers are quite thermostable, and reactions of their thermal decomposition proceed only at relatively high temperatures

$$
\begin{aligned}
\mathrm{Fe}(\mathrm{OH})_{2} & =\mathrm{FeO}+\mathrm{H}_{2} \mathrm{O}_{\text {(vapour) }} 423-473 \mathrm{~K}, \\
2 \mathrm{FeOOH} & =\mathrm{Fe}_{2} \mathrm{O}_{3}+\mathrm{H}_{2} \mathrm{O}_{\text {(vapour) }} 773-973 \mathrm{~K}, \\
2 \mathrm{FeOOH}+\mathrm{Fe}(\mathrm{OH})_{2} & =\mathrm{Fe}_{3} \mathrm{O}_{4}+2 \mathrm{H}_{2} \mathrm{O}_{\text {(vapour) }} 873-1273 \mathrm{~K} .
\end{aligned}
$$

Therefore, electrochemical oxidation of the discussed minimally charged iron metal nanoparticle is the process that can effectively compete with the process of oxidation of this nanoparticle by neutral oxidizing species even at quite high temperatures.

At such temperatures, hydrogen gas evolved in exothermal reaction (44), can be auto-ignited directly on the surface of the discussed aerosol nanoparticle in humid air, because an autoignition temperature of the air-hydrogen gas mixtures is about $858 \mathrm{~K}$ (i.e., $585^{\circ} \mathrm{C}$ ). Preferential electrochemical oxidation of the continuously electroydrated charged iron nanoparticles in humid air will convert these nanoparticles into the short-circuited iron/air core-shell nanobatteries, or more precisely into the short-circuited iron metal/iron oxy-hydroxide/water vapour nanobatteries, that is, into the short-circuited core-shell nanobatteries with the iron metal-based core reductant, with the iron hydroxide/iron oxy-hydroxide-based porous electrolyte shell, and with the external gas oxidant-that is, predominantly with water vapour (Figure 10). 


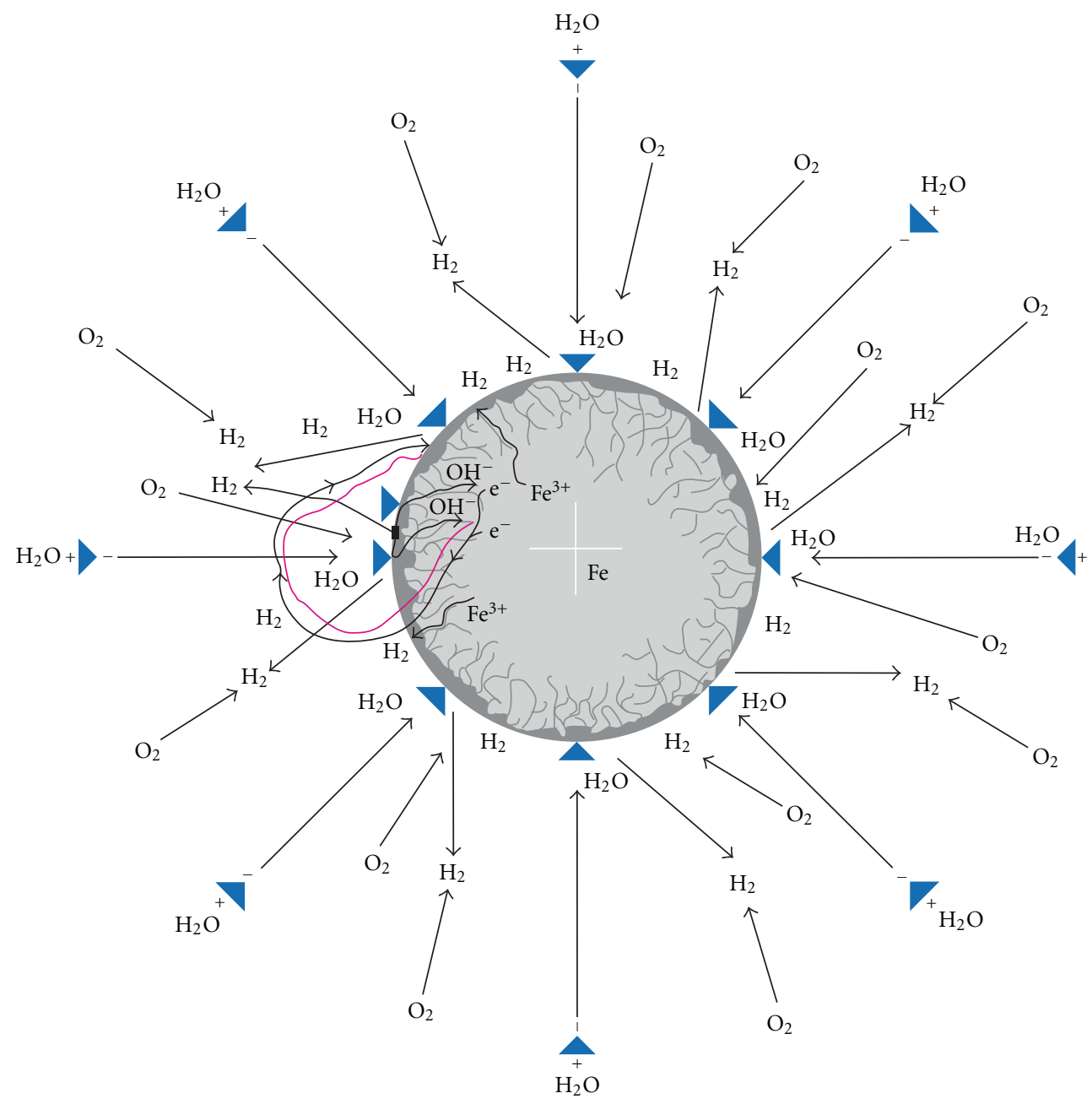

A core reductant of the nanoparticle, for example, $\mathrm{Fe}, \mathrm{Al}, \mathrm{Cu}, \mathrm{W}, \mathrm{Si}, \ldots$

A growing shell electrolyte of the nanoparticle, for example, $\mathrm{FeOOH}, \mathrm{Fe}(\mathrm{OH})_{3}, \mathrm{Fe}(\mathrm{OH})_{2}$, $\mathrm{AlOOH}, \mathrm{Al}(\mathrm{OH})_{3}, \mathrm{Cu}(\mathrm{OH})_{2}$, tungstic acids, silicic acids, ...

A field-emission and/or thermionic repetitive break-down

$\sum$ through the hydrated porous electrolyte nanolayer

FIGURE 10: The electrostatic charge of combustible nanoparticles can be a powerful catalyst of their intense oxidation by polar molecules of water vapour in a humid atmosphere. Due to the additional energy of electrostatic acceleration, polar molecules of water vapour can actively oxidize various highly charged nanoparticles, including nanoparticles consisting of materials, which are relatively inert at temperatures of $\leq 700 \mathrm{~K}$ (e.g., such as soot and other carbon-based nanoparticles that, when uncharged, are inert in humid air at these temperatures). During the process of such charge-catalyzed humid air oxidation of combustible nanoparticles by surrounding water vapour molecules, various combustible gases can be generated (e.g., hydrogen gas when oxidizing nanoparticles consisting of some reactive metals; or a mixture of hydrogen gas and carbon monoxide when oxidizing some carbon-containing nanoparticles; or hydrogen sulphide gas when oxidizing some metal sulphide nanoparticles). Repeating processes of auto-ignition of evolved combustible gases can accompany water vapour-induced oxidation of charged combustible nanoparticles in humid air. When the autoignition of the combustible gases takes place within such a cloud, a flame is not always visible. In the case of the water vapour induced oxidation of charged metal/metalloid nanoparticles, for example, the iron or aluminium or silicon ones, the evolved hydrogen gas can be auto-ignited without a visible flame.

Thus, the charged iron metal nanoparticle can be spontaneously transformed into the nanoscale battery with an internal reductant-iron metal core-an anode of the battery. During the process of humid air oxidation, the internal iron metal anode is periodically emitting electrons outwards, through the porous surface electrolyte layers, to the outer surface of the nanoparticle, that is, to a virtual cathode of this core-shell nano capsule-nanobattery.

The diffusion flux of the oxidizable iron-containing ions, that is, $\mathrm{Fe}^{2+}, \mathrm{Fe}^{3+}, \mathrm{Fe}(\mathrm{OH})^{+}$, or $\mathrm{Fe}(\mathrm{OH})^{2+}$, also moves 
from the iron metal core through the $\mathrm{FeOOH} / \mathrm{Fe}(\mathrm{OH})_{2}$ containing surface electrolyte layers to the outer surface of the charged iron metal nanoparticle. These positively charged iron-containing ions are exposed to oxidation on and within the hydroxide-based nanoparticle shell where these ions meet and recombine with electrons that are periodically emitted from iron metal core (i.e., only when the electrochemically generated intra-particle electrostatic intensity reaches limiting values permitting field and/or thermionic electron emission from the iron metal core.

One of numerous possible oxidative reactions, which can take place directly on the hydrated iron hydroxide based surface of the charged iron metal nanoparticle-nanobattery, with the participation of the surrounding electrostatically accelerated water vapour molecules and the oxidizable ironcontaining ions

$$
\mathrm{Fe}(\mathrm{OH})^{2+}{ }_{\text {(ion) }}+2 \mathrm{e}+2 \mathrm{H}_{2} \mathrm{O}_{\text {(vapour })} \longrightarrow \mathrm{Fe}(\mathrm{OH})_{3}+\mathrm{H}_{2} .
$$

The counter diffusion flux of negative hydroxyl ions through the $\mathrm{FeOOH} / \mathrm{Fe}(\mathrm{OH})_{2}$ containing surface electrolyte layers transports new electrons to the nanoparticle iron metal core also contributing to an additional negative charging of the iron metal core.

The hydroxyl ions that combine with the iron metal core will oxidize the iron and leave additional electrons on this metal core of the nanoparticle. These diffusion processes, involving hydroxyl ions, will also provoke the subsequent field and/or thermionic electron emission intraparticle breakdowns.

In humid air, an iron metal nanoparticle, being even uncharged with a surplus charge, can still be electrostatically hydrated by surrounding water vapour molecules because of its initial oxygen-induced oxidative charging due to electron diffusion from the iron metal core to the growing semiconductor $\mathrm{FeO} / \mathrm{Fe}_{3} \mathrm{O} 4$ based-shell (Figure 11).

During electrochemical oxidation of the charged iron metal nanoparticle, quick-alternating electric dipole moments and huge potential differences can arise within the nanoparticle between the randomly arising local oxidative spots of positive charges on the outer surface of the nanoparticle and the corresponding uncompensated residual negative charges of the iron metal core.

Successive random "Brownian" collisions of the nanoparticle with surrounding gas molecules will not only continuously and irregularly change the coordinates of the aerosol nanoparticle but also, in a random manner, they will change the instantaneous values of the electric dipole moment of the nanoparticle because of the stochastic, "spotty", nature of the nanoparticle oxidation.

The electrochemically generated fluctuating electric dipole moments, continuously arising within the nanoparticles-nanobatteries during oxidation, will cause a powerful electric dipole-dipole attraction between the separate aerosol nanoparticles-nanobatteries within a cloud of such nanobatteries. At the same time, the huge intraparticle core-shell voltage, electrochemically generated, will also cause repeating processes of the field and/or thermoionic electron emission breakdowns from the iron metal core to the outer surface of the nanoparticle during oxidation.

As these core-shell nanobatteries, spontaneously generated from the combustible iron metal aerosol nanoparticles during their oxidation, can be exposed to such continuously repeating relaxation processes (i.e., the intra-particle electron emission breakdowns), these periodically short-circuited aerosol nanoparticles-nanobatteries can be also described as pulsating aerosol current loops [20].

The strong alternating currents and corresponding magnetic moments generated within the periodically shortcircuited aerosol nanobatteries can cause an additional powerful magnetic dipole-dipole attraction within a cloud of such nanobatteries.

Within a ball lightning cloud, despite the presence of unipolar charges on the surface of many aerosol nanoparticles-nanobatteries, both the electric and magnetic dipole-dipole attractions take place between the unipolar charged nanoparticles-nanobatteries. Both these alternating types of electromagnetic dipole-dipole attraction between charged aerosol nanobatteries contribute to significant dipole-dipole electromagnetic cohesion of the aerosol substance of such a cloud. (Certainly, the greater part of surplus electrostatic charges of the nanoparticles-nanobatteries within ball lightning will be probably localized on the peripheral aerosol nanoparticles due to mutual Coulomb repulsion between these unipolar charged nanoparticles and also due to a minimal air electroconductivity.)

Reaction (43)

$$
\begin{aligned}
& 3 \mathrm{Fe}_{(\text {nanoparticle })}+6 \mathrm{H}_{2} \mathrm{O}_{\text {(vapour) }} \\
& \quad=\mathrm{Fe}(\mathrm{OH})_{2}+2 \mathrm{FeO}(\mathrm{OH})+4 \mathrm{H}_{2} \uparrow
\end{aligned}
$$

is only slightly exothermal, with a moderate heat-evolution $\sim 150 \mathrm{~kJ}$ per one mol of the produced $\mathrm{Fe}(\mathrm{OH})_{2}$. However, a concomitant auto-ignition and combustion of the evolved hydrogen gas in ambient air could give considerable additional heat and water vapour

$$
4 \mathrm{H}_{2 \text { (gas) }}+2 \mathrm{O}_{2 \text { (gas) }}=4 \mathrm{H}_{2} \mathrm{O}_{(\text {vapour })}-968 \mathrm{~kJ} / \mathrm{mol} .
$$

The repetitive processes of heating the nanoparticlesnanobatteries, caused by the repetitive processes of heatevolution from reactions (36) and (39), can periodically and probably with a high frequency dehydrate the $\mathrm{FeOOH} / \mathrm{Fe}(\mathrm{OH})_{2}$-based surface electrolyte layers, which grow on these iron metal nanoparticles, and so this thermal nanoparticle dehydration will periodically inhibit the process of electrochemical oxidation of the charged iron metal nanoparticles with a temporary conversion of the iron hydroxide based surface electrolyte layers into the dehydrated, $\mathrm{Fe}_{3} \mathrm{O}_{4}$-based, semi-conducting surface layers of mixed iron oxides (Figure 12).

However, a fast electrostatic rehydration of the charged iron metal aerosol nanoparticle by surrounding polar molecules of water vapour in humid air will contribute to immediately cooling this combustible nanoparticle, and so the process of predominantly electrochemical, ionmediated oxidation of the iron nanoparticle through its 


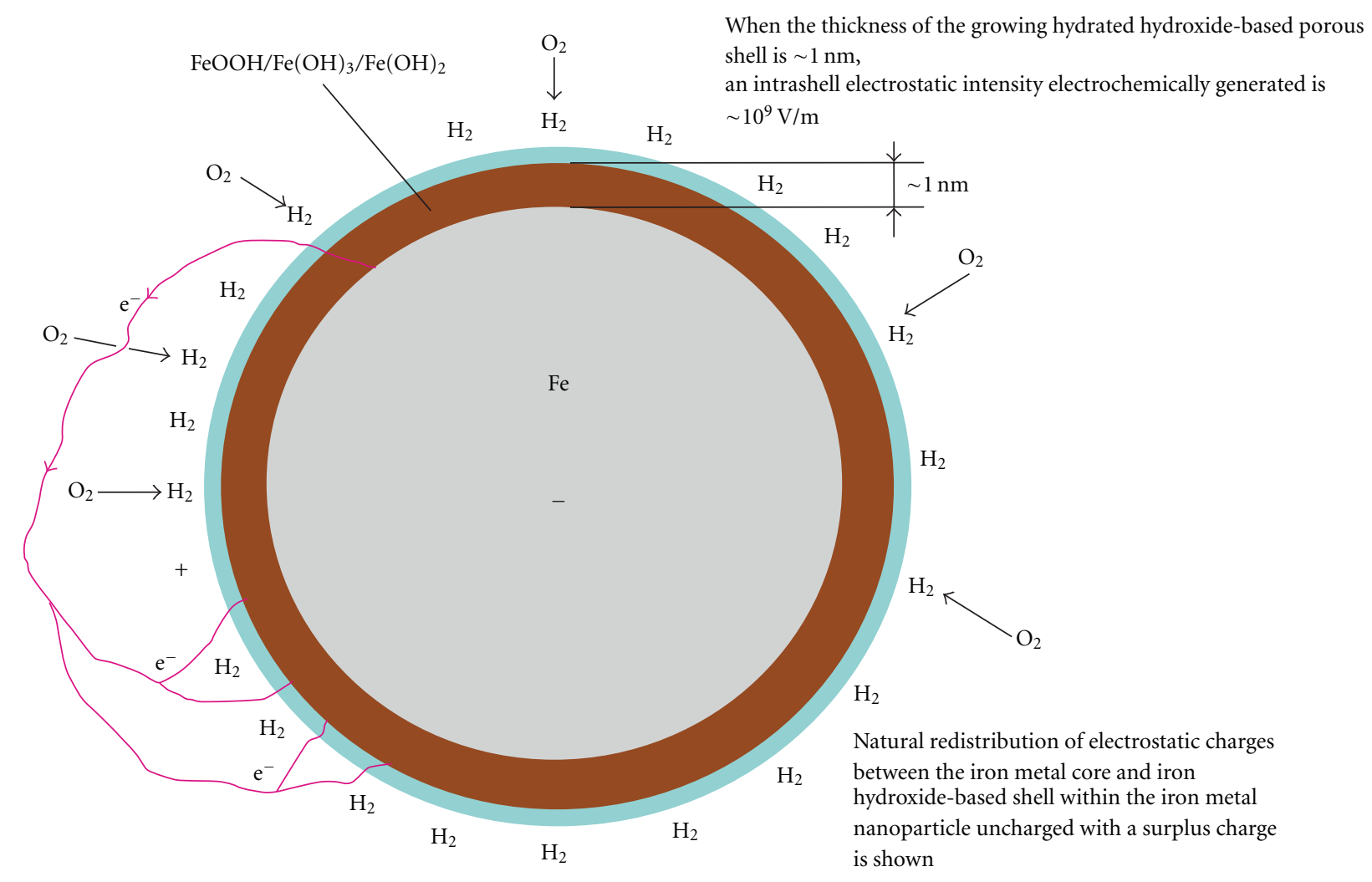

Dynamic layer of the absorbed

molecules of water vapor

This iron metal nanoparticle is predominantly oxidized by water vapour molecules

Dynamic porous layer/shell of the

hydrated hydroxide electrolytes

An oxidizable core of nanoparticle

FIGURE 11: When electrostatically neutral metal aerosol particle is exposed to a gradual spotty oxidation in humid air, it is initially covered with a semi-conductor or dielectric metal oxide shell. Correspondingly, electron diffusion from the metal core into the semiconductor or dielectric shell will charge the metal core with a positive charge, while the metal oxide shell of this particle will be spotty charged with a negative charge. Local spots of the negative charge will arise on the surface of this particle during its initial oxidation. These locally charged oxidized surface spots will attract surrounding polar molecules of water vapour from ambient air due to a charge-dipole interaction. So, the particle surface will be quickly and completely hydrated. Such electrostatic hydration of the nanoparticle surface can often transform the metal oxide semiconductor or dielectric shell into the metal hydroxide or oxyhydroxide electrolyte shell. In this case, at relatively low temperatures, preferential diffusion of the metal or hydroxyl ions within the hydrated electrolyte surface layers will form a new, opposite redistribution of electrostatic charges between the metal core (negatively charged now) and electrolyte shell (positively charged) with periodic relaxation of such electrochemically generated increasing electrostatic intensity by the field and thermoionic electron emission core-shell breakdowns.

rehydrated $\mathrm{FeOOH} / \mathrm{Fe}(\mathrm{OH})_{2}$ based electrolyte shell will be reactivated again. Probably, such self-oscillating processes of electrochemical oxidation of the charged combustible nanoparticles, continuously electrostatically re-hydrated by surrounding molecules of water vapour, could be quite high-frequency due to the extremely low heat capacity and the extremely high specific surface area of the aerosol nanoparticles.

These oxidative self-oscillating thermocycling processes can be partially (or totally) synchronized between all the charged aerosol nanoparticles-nanobatteries within ball lightning. Probably, such synchronization of the selfoscillating oxidative processes within ball lightning could also contribute to similar synchronization of the thermoionic electron emission processes of nanobatteries' short circuits with corresponding generation of powerful coherent radio frequency radiation by such a ball lightning cloud.

Within a ball lightning cloud, possibly, there is another mechanism in order that to synchronize an alternation of the stages of electrostatic oxidative hydration of nanoparticlesnanobatteries with the stages of electron emission breakdowns within these nanobatteries. The collective electron emission breakdowns of the aerosol nanobatteries within ball lightning are probably able to generate a wideband radio frequency radiation. At the same time, radio frequency radiation with resonant wavelengths, which are correlated with the specific diameter of the specific lightning ball, could become most strong. If a ball lightning nanobattery cloud 


\begin{abstract}
A highly charged unoxidized iron metal aerosol nanoparticle suspended in humid air
\end{abstract}
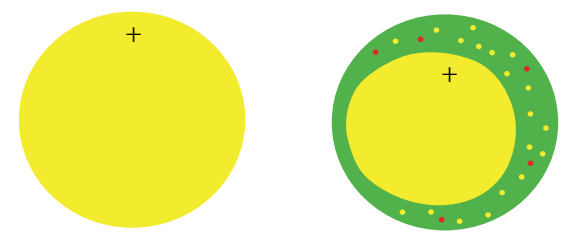

First step of a possible self-oscillating process of water vapor oxidation of a highly charged iron nanoparticle,

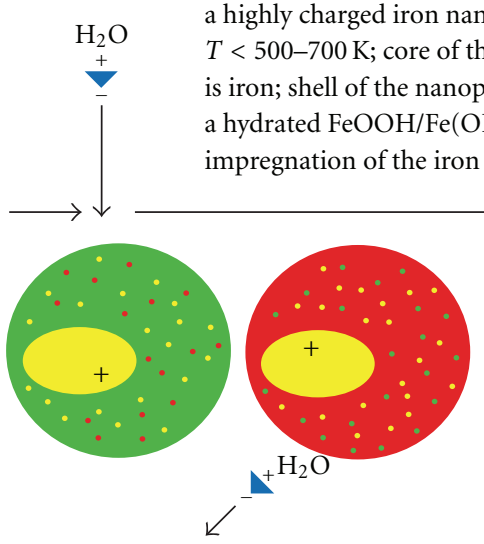

FIgURE 12: The stages of stepped exothermal oxidation of iron metal nanoparticles by surrounding polar molecules of water vapour successively alternate with stages of thermal dehydration of these nanoparticles to periodically convert the $\mathrm{FeOOH} / \mathrm{Fe}(\mathrm{OH})_{2}$ based electrolyte shells of the nanoparticles into the $\mathrm{Fe}_{3} \mathrm{O}_{4} / \mathrm{Fe}_{2} \mathrm{O}_{3}$ based semiconducting shells. Electrostatic oxidative adsorption of water vapour molecules forms a hydroxide based site on the charged nanoparticle surface. Such periodic oxidative electroadsorption of water vapour is accompanied by local evolving hydrogen gas. The hydrogen gas can autoignite in humid air, heating the nanoparticle and so again dehydrating it. These electrostatic oxidative hydration/thermal dehydration alternating processes will repeat time and again. A final reaction product will consist of almost completely oxidized nanoparticles. The alternating stages of the nanoparticle's hydration/dehydration are accompanied by a successive change of electrophysical characteristics of the core/shell junctions within the nanoparticles, from the iron metal/iron hydroxide electrolyte junctions to iron metal/iron oxide semiconductor junctions, and then in the opposite direction. These periodic changes of the nanoparticle's surface characteristics can cause a successive intra-particle electron-ion transfer. Thus, the surface of the charged metal nanoparticle can continuously modify its composition and structure from metal oxide into metal oxy-hydroxide into metal hydroxide, then in the opposite direction and so forth, through such a self-oscillating thermocycling process of nanoparticle's humid air oxidation. An alternating electrostatic intensity of $\sim$ one billion volts/metre will be generated inside the surface layers at each new stage of their repeating electrostatic hydration or thermal hydration. Correspondingly, the field or thermionic electron emission intraparticle breakdowns will occur at each stage of such reincreasing of core/shell electrostatic intensity.

is predominantly resonant self-oscillating radio frequency generator with the inner oxidative energy source, such model of the self-sustaining nanoparticle-based resonant radio frequency aerosol generator can be considered as a similar and, at the same time, inverse model in relation to the theoretical and experimental mechanisms described in [5, 6, 34-38]. Within ball lightning, the collective short circuits of trillions of the charged nanobatteries could generate the self-coordinated resonant oscillations (namely, the very high frequency or ultra-high frequency or super-high frequency oscillations according to the typical ball lightning diameters). Such resonant electromagnetic oscillations probably could in turn synchronize the successive intra-cloud processes of the periodic total dehydration, total heating, and total thermoionic electron emission breakdowns within all or almost all the aerosol nanobatteries. A presence of such electromagnetic negative feedback within the cloud of the electrostatically charged and periodically short-circuited nanobatteries could help to understand a normal constancy of the ball lightning diameter during its life time if certainly indeed ball lightning is a self-oscillating resonant radio frequency generator.

So, oxidation of the charged iron nanoparticles in humid air is a predominantly water vapour induced process. 
Correspondingly, this oxidative process can proceed predominantly through electrochemical, that is, ion-mediated mechanism with formation of hydroxide or oxy-hydroxide or hydroxo-carbonate-based nanoporous electrolyte layers on the charged nanoparticle's surface and evolving potentially inflammable hydrogen gas. Correspondingly, this humid air oxidative process can be accompanied by periodic autoignition of the evolved hydrogen gas and so this process can have a self-oscillating thermocycling character with significant temperature pulsations.

With reference to the problem of ball lightning, it would also be interesting to find out whether minimally charged aluminium aerosol nanoparticles can be exposed to a similar strong electrostatic attack by accelerated highreactive surrounding polar molecules of water vapour in humid air.

\section{Humid Air Oxidation of a Spherical $(\sim 2 \mathrm{~nm}$ in Diameter) Aluminium Metal Aerosol Nanoparticle Charged with the Minimum Positive Charge $Q=|e|=1.6 \cdot 10^{-19}(\mathbf{C})$ at the Aluminium Melting-Point of about $933 \mathrm{~K}$.}

Let us again suppose that in humid air the mole fraction of molecules of water vapour, $n_{W}$, is $\sim 0.02$. Let us also suppose that at aluminium's melting-point of $\sim 933 \mathrm{~K}$ (i.e., $\sim 660^{\circ} \mathrm{C}$ ), an average value of both activation energies, $E_{a-A l}(\mathrm{~J} / \mathrm{mol})$, for the competing processes of water vapour and oxygen gas induced oxidation of aluminium metal (i.e., an average value of the activation energy for the process of water vapour induced oxidation of an aluminium nanoparticle, $E_{a W-A l}$ $(\mathrm{J} / \mathrm{mol})$, and the activation energy for the process of oxygen gas induced oxidation of this nanoparticle $E_{a O-A l}(\mathrm{~J} / \mathrm{mol})$ can be of $\sim 200,000(\mathrm{~J} / \mathrm{mol})$ [39]

$$
E_{a-A l} \approx E_{a W-A l} \approx E_{a O-A l} \sim 200,000(\mathrm{~J} / \mathrm{mol}) .
$$

Thus, at $T \approx 933 \mathrm{~K}$ :

$$
\begin{aligned}
k T & \approx 1.38 \cdot 10^{-23} \cdot 933=1.29 \cdot 10^{-20}(\mathrm{~J}), \\
\mathcal{E}_{a-A l} & =\frac{E_{a-A l}}{N A} \approx \frac{200,000}{\left(6.02 \cdot 10^{23}\right)} \approx 3.3 \cdot 10^{-19}(\mathrm{~J}),
\end{aligned}
$$

where $N_{A} \approx 6.02 \cdot 10^{23}$ - the Avogadro constant $\left(\mathrm{mol}^{-1}\right)$.

Consequently, in the case of humid air oxidation of the minimally charged aluminium aerosol nanoparticle at aluminium's melting point

$$
\begin{gathered}
\frac{\varepsilon_{a-A l}}{k T}=25.6 \\
\frac{\varepsilon_{a-A l}}{(k T+\varepsilon 1)}=\frac{3.3 \cdot 10^{-19}}{\left(2.15 \cdot 10^{-20}\right)}=15,35
\end{gathered}
$$

Let us again recall that $\varepsilon_{1} \approx 8.6 \cdot 10^{-21}(\mathrm{~J})$ is the additional average kinetic energy acquired by the polar molecule of water vapour in the electrostatic field of a minimally charged nanoparticle at a distance of the mean free path from the nanoparticle.
Thus, the fraction of the reactive oxygen gas molecules, that is oxygen gas molecules possessing enough kinetic energy to climb the activation energy barrier of the oxygen gas induced oxidative reactions on the surface of the discussed aluminium nanoparticle at a temperature of about $933 \mathrm{~K}$, is

$$
\begin{aligned}
\frac{\Delta N_{O}}{N_{O}} & =\frac{2}{\sqrt{\pi}} \int_{25.6}^{\infty} \sqrt{\varepsilon^{\prime}} e^{-\varepsilon^{\prime}} d \varepsilon^{\prime} \\
& \approx \frac{2}{\sqrt{\pi}} e^{-25.6}\left(\sqrt{25.6}+\frac{1}{2 \sqrt{25.6}}\right)=4.44 \cdot 10^{-11} .
\end{aligned}
$$

Consequently, the competing fraction of the reactive water vapour molecules possessing enough kinetic energy to climb the activation energy barrier of the water vapour induced oxidative reactions on the surface of the discussed minimally charged aluminium nanoparticle at a temperature of about $933 \mathrm{~K}$ is

$$
\begin{aligned}
\frac{\Delta N_{H}}{N_{H}} & =\frac{2}{\sqrt{\pi}} \int_{15.35}^{\infty} \sqrt{\varepsilon^{\prime}} e^{-\varepsilon^{\prime}} d \varepsilon^{\prime} \\
& \approx \frac{2}{\sqrt{\pi}} e^{-15.35}\left(\sqrt{15.35}+\frac{1}{2 \sqrt{15.35}}\right)=9.89 \cdot 10^{-7} .
\end{aligned}
$$

Thus, in this relatively high temperature case of oxidation of the minimally charged aluminium nanoparticle:

(a) the absolute quantity of the reactive non-polar molecules of oxygen gas, which is able to climb the activation energy barrier of the oxidative reactions on the surface of the discussed minimally charged nanoparticle at a temperature of about $933 \mathrm{~K}$, and which are contained in one mole of humid air

$$
\begin{aligned}
\Delta N_{O-A l} & =N_{O} \cdot 4.44 \cdot 10^{-11} \\
& =1.26 \cdot 10^{23} \cdot 4.44 \cdot 10^{-11}=5.6 \cdot 10^{12} .
\end{aligned}
$$

(b) the absolute quantity of the reactive polar molecules of water vapour, which is able to climb the activation energy barrier of the oxidative reactions on the surface of the discussed minimally charged nanoparticle at a temperature of about $933 \mathrm{~K}$, and which are contained in one mole of humid air

$$
\begin{aligned}
\Delta N_{H-A l} & =N_{H} \cdot 9.89 \cdot 10^{7} \\
& =1.2 \cdot 10^{22} \cdot 9.89 \cdot 10^{-7}=1.19 \cdot 10^{16} .
\end{aligned}
$$

As one can see, the number of reactive water vapour molecules, which are able to oxidize the discussed minimally charged molten aluminium nanoparticle suspended in humid air at a temperature of $\sim 933 \mathrm{~K}$ is

$$
\frac{\Delta N_{H-A l}}{\Delta N_{O-A l}}=\frac{1.19 \cdot 10^{16}}{5.6 \cdot 10^{12}}=2.1 \cdot 10^{3}
$$


times larger than the number of reactive oxygen gas molecules, which are able to oxidize the charged surface of this nanoparticle under such relatively high-temperature conditions when the mole fraction of competing water vapour molecules, $n_{W}$, in humid air is $\sim 0.02$.

In this case, also an unexpectedly large (approximately two thousand times) difference is found between the number of reactive water vapour molecules, which are able to oxidize the minimally charged aluminium nanoparticle in humid air and the number of reactive molecules of oxygen gas, competitively participating in this humid air oxidation. This means that relatively few polar molecules of water vapour in humid air, being additionally electrostatically accelerated by their charge-dipole attraction towards the minimally charged aluminium nanoparticle, become practically the only essential air oxidant for the discussed minimally charged aluminium nanoparticle, and non-polar molecules of oxygen gas actually do not take part in humid air oxidation of this charged nanoparticle even at a temperature of aluminium melting of $\sim 933 \mathrm{~K}$.

Thus, probably, that one of the main oxidative reactions on the surface of the minimally charged aluminium nanoparticle will be a water vapour-induced exothermal reaction of oxidation of aluminium metal with formation of the hydrated, aluminium hydroxide $\mathrm{Al}(\mathrm{OH})_{3}$ and/or aluminium oxy-hydroxide $\mathrm{AlOOH}$ based porous electrolyte surface layers with synchronous evolution of hydrogen gas

$$
\begin{aligned}
& 2 \mathrm{Al}_{(\text {nanoparticle })}+6 \mathrm{H}_{2} \mathrm{O}_{(\text {vapour })} \\
& \quad=2 \mathrm{Al}(\mathrm{OH})_{3(\text { nanoparticle shell })}+3 \mathrm{H}_{2} \uparrow \\
& \mathrm{Al}(\mathrm{OH})_{3(\text { nanoparticle shell })} \\
& \quad=\mathrm{AlOOH}_{(\text {nanoparticle shell })}+\mathrm{H}_{2} \mathrm{O}_{(\text {vapour })} \leq 200^{\circ} \mathrm{C}
\end{aligned}
$$

$2 \mathrm{AlOOH}_{(\text {nanoparticle shell) }}$

$$
=\mathrm{Al}_{2} \mathrm{O}_{3 \text { (nanoparticle shell) }}+\mathrm{H}_{2} \mathrm{O}_{(\text {vapour })} \geq 420^{\circ} \mathrm{C} \text {. }
$$

In the case of such predominantly water vapour induced oxidation of the charged aluminium aerosol nanoparticles, the solid reaction products, growing on the surface of these nanoparticles mainly in the form of the thermolabile hydrated aluminium oxy-hydroxide-based electrolyte layers saturated with hydrogen, will limit diffusion and direct oxidation of these aluminium nanoparticles by external neutral oxidizing species, for example such as oxygen gas, but at the same time, these electrolyte shells will effectively transport ionized species, such as $\mathrm{Al}^{3+}$ or $\mathrm{Al}(\mathrm{OH})^{2+}$ or hydroxyl ions, contributing to the preferential electrochemical mechanism of oxidation of the charged aluminium nanoparticles.

One of numerous possible oxidative reactions, which could take place directly on the hydrated aluminium hydroxide based surface of the charged aluminium metal nanoparticle-nanobattery, with the participation of the surrounding electrostatically accelerated water vapour mole- cules and the oxidizable aluminium-containing ions:

$$
\mathrm{Al}(\mathrm{OH})^{2+}{ }_{\text {(ion) }}+2 \mathrm{e}+2 \mathrm{H}_{2} \mathrm{O}_{\text {(vapour) }} \longrightarrow \mathrm{Al}(\mathrm{OH})_{3}+\mathrm{H}_{2} \text {. }
$$

Thus, in humid air, minimally electrostatically charged aluminium metal-based aerosol nanoparticles might be oxidized by surrounding water vapour molecules rather than by non-polar oxygen gas molecules even at the aluminium melting point $\sim 933 \mathrm{~K}$. Such processes of intense electrostatic hydration and corresponding predominantly water vapour induced oxidation of the charged aerosol molten aluminium nanodroplets can automatically convert these nanoparticles into aluminium/air core-shell nanobatteries with the aluminium core anodes and the aluminium oxy-hydroxide based porous electrolyte shells, which play the role of airdepolarized cathodes of such aluminium/air nanobatteries.

As mentioned above, numerous electron emission breakdowns could probably occur within a cloud consisting of the electrostatically charged metal, metalloid, or carbon based nanobatteries, for example within aluminium metal based ball lightning, during preferential electrochemical oxidation/burning of such charged nanoparticles in humid air. Even only partially synchronized intra-particle electron breakdowns within ball lightning consisting of trillions of aerosol nanobatteries could generate a powerful radiofrequency radiation, including microwave radiation.

It seems that a possible generation of radio frequency radiation over the range of $\sim 0.1$ gigahertz up to $\sim 20$ gigahertz from burning aerosol clouds consisting of charged combustible particles could be found in corresponding aerosol combustion experiments. Probably, a condensed disperse phase of such radio frequency emitting slowly burning aerosol clouds could consist of bipolar or unipolar charged nano or submicron combustible particles, e.g., such as aluminium-, or iron-, or magnesium-, or zirconiumbased nanoparticles, or also carbon- or silicon-based combustible nanoparticles co-aggregated with additional electrolyte nanoparticles. In standard humid air, predominantly electrochemical burning of such electrostatically charged nanoparticles could be accompanied with intense thermoemission and ultra-violet induced photoemission charging processes, sustaining spontaneous electrostatic charging of these burning nanoparticles.

In particular, the formation of electrochemically burning aerosol clouds, capable of generating powerful radio frequency radiation, could be spontaneously realized in various hypervelocity flight processes, for example in a troposphere movement of iron-nickel-based meteors or in a flight of hypervelocity rockets. During the troposphere movement of hypervelocity objects, aerosol clouds consisting of the short-circuited nanobatteries can be produced either by high-temperature ablation of heat-insulating materials or by incomplete combustion of carbon based fuels. The presence of combustion-generated water vapour, highly charged soot nanoparticles and additional electrolyte impurities in plasma-condensation trails of hypervelocity rockets can probably contribute to activation of a charge-catalyzed water vapour induced electrochemical oxidation of these soot 
nanoparticles-nanobatteries with a resultant generation of powerful radiointerference.

\section{Some Final Remarks}

Clearly, the discussed effect of the charge-catalyzed water vapour-induced oxidation of unipolar and/or bipolar, electrostatically or electrodynamically charged aerosol nanoparticles/substrate-integrated nanostructures can take place in the processes of humid air oxidation of various combustible nanoobjects, not only metal or metalloid ones, as the large additional kinetic energy, acquired by surrounding polar molecules of water vapour in the electrostatic field of the charged nanoparticles at a distance of their mean free path from the nanoparticles, is independent of the materials constituting these nanoobjects. A change in polarity of the surplus charge of the combustible nanoparticles is also not able to change the value of the additional kinetic energy acquired by surrounding polar gas molecules in the electrostatic field in immediate proximity to the charged nanoparticles.

Correspondingly, combustible particles charged with equal, either negative or positive, electrostatic charges can be equally intensively attacked by surrounding water vapour molecules accelerated in the electrostatic field of these nanoparticles, absolutely irrespective of the polarity of the nanoparticle's surplus charges.

In many cases, in humid air, such an intense chargecatalyzed water vapour induced oxidation of the charged metal or metalloid nanoparticles can mainly proceed through the electrochemical mechanism just due to the preferential growth of the hydrated hydroxide based dynamic electrolyte surface layers, resaturated with hydrogen gas. These thermally unstable, continuously renewed and again decomposed, passivating surface electrolyte layers can significantly inhibit further oxidation of combustible nanoparticles by gas phase neutral oxidizing species. At the same time, these surface electrolyte layers will stimulate the relatively low-temperature process of the intra-particle ion diffusion and ion-mediated oxidation of the combustible nanoparticles. A time-average temperature of such electrochemical oxidation of the combustible nanoparticles can be relatively low, probably ranging from $\sim 300$ to $900^{\circ} \mathrm{C}$, particularly taking into account the above-described scenario of the selfoscillating electrochemical oxidation with the alternating thermocycling processes of oxidative electrostatic hydration and thermal flame dehydration of the combustible particles.

According to [20], when an average diameter of the soot-based aerosol nanoparticles-nanobatteries constituting a soot-based ball lightning cloud is $\sim 100 \mathrm{~nm}$ and when a total mass of this condensed phase of the ball lightning cloud is $\sim 4$ grammes, then the average number of such carbon/air nanoparticles-nanobatteries can be of $\sim 10^{15}$ in this $\sim 20 \mathrm{~cm}$ diameter ball lightning cloud. If a net electrostatic charge of such "average" ball lightning ranges at least from $\sim 0.1$ to 1 microcoulomb, that is, from $\sim 6.2 \times 10^{11}$ to $\sim 6.2 \times$ $10^{12}$ elementary charges, then large aerosol nanoaggregates consisting of $\sim 200-2.000$ such nanoparticles can be charged only with a single surplus mobile ion per one large nanoaggregate (or also with a single surplus mobile ion per one submicron- or micrometre-sized large compact combustible aerosol particle).

In any case, it is evident that combustible aerosol particles constituting ball lightning can only have $\sim$ one surplus ion per one aerosol particle on average. Correspondingly, within ball lightning, the charge-catalyzed water vapour induced oxidation of combustible particles will practically always proceed through the step-by-step successive mechanisms, which are illustrated in the Figures (2-5). Equally, it is clear that a high hydrophility of the oxidized surface of these combustible nanoparticles (the high hydrophility is a typical property of the overwhelming majority of metal oxides, metal oxy-hydroxides, and metal hydroxides) could enable a fast redistribution of locally electroadsorbed water vapour molecules over all the reacting surface of such large, submicron- or micrometre-sized, either aggregated or compact, combustible aerosol particles.

Probably, a lot of the combustible aerosol particles constituting the ball lightning cloud are in fact uncharged. At the same time, within ball lightning these particles are strongly polarized, so these oxidizable particles can still be exposed to an intense local charge-catalyzed water vapour induced oxidation.

For the sake of brevity, a lot of important additional aspects cannot be considered in this paper. Although discussion of certain additional aspects, such as a temperaturedependent core-shell jumping of mobile surplus charges inside the nanoparticles, the effect of the polarity, mobility and intra-particle location of the surplus ions on the rate of electrochemical oxidation of the combustible nanoparticles, the charge-dependent activated "anodic" oxidation of negatively multiply charged combustible nanoparticles or, on the contrary, the charge-dependent inhibited oxidation of positively multiply charged combustible nanoparticles ("cathodic protection") - the effects, which are somewhat similar to those described in $[40,41]$, could be also useful, all these important aspects fall outside the limits of this paper. At the same time, various possible versions of electrochemical redistribution of charges inside some metal aerosol nanoparticles, that could spontaneously form the shortcircuited metal/air aerosol nanobatteries, minimally charged and hence actively electrostatically hydrated in humid air, are shown in the Figures 13, 14, 15, 16, 17 and 18.

The additional kinetic energy of the electrostatic acceleration acquired by polar gas molecules near to a charged nanoparticle is independent not only of the specific material components of the charged nanoparticle and the polarity of the nanoparticle's surplus charge, but also of the presence and type of chemical reactions on the surface of the nanoparticle.

This means that the substantial additional kinetic energy of $\geq 10^{-20}(\mathrm{~J})$, which can be acquired by various electrostatically accelerated polar gas molecules in immediate proximity to charged aerosol nanoparticles (or charged surfaceintegrated nanostructures), could contribute to powerful catalysis of various heterophase reactions, not only oxidative 


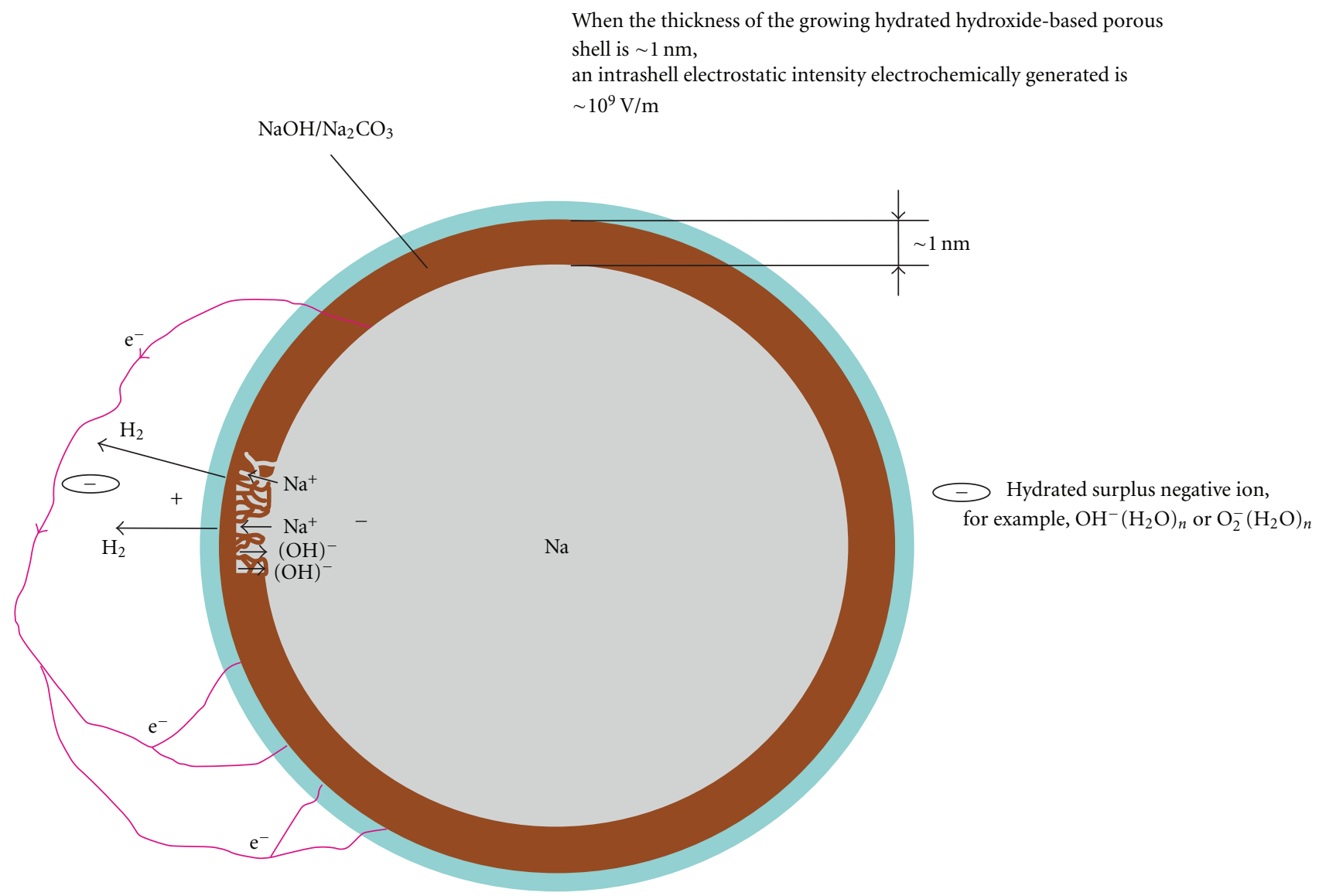

\footnotetext{
Dynamic layer of the absorbed molecules of water vapor Dynamic porous layer/shell of the hydrated hydroxide electrolytes
}

An oxidizable core of nanoparticle

FIGURE 13: In humid air, negatively charged sodium metal based nanoparticles or their aggregates with soot carbon nanoparticles are continuously re-electrohydrated by surrounding molecules of water vapour, and so they can be spontaneously transformed into the sodium/air or aggregated composite sodium-carbon/air core-shell nanobatteries, which are periodically short-circuited by the intraparticle core-shell electron emission breakdowns. Probably, the small ball-shaped clouds of such sodium/air core-shell nanoparticles-nanobatteries could be generated by high-voltage pulse-arc electrolysis from the $\mathrm{NaHCO}_{3}$ containing water solutions in the experiments [4, 7]. At an initial stage, the sodium metal and hydrogen gas could be synchronously produced on the hot carbon cathode. In these experiments, milligrammes of the fresh-reduced electrolysis-generated sodium metal could be immediately evaporated by the pulse arc discharge. Then, the evaporated sodium metal can be condensed in the form of the small cloud of the negatively charged sodium metal nanoparticles in the local hydrogen gas based reducing atmosphere. In humid air, predominantly electrochemical oxidation of the highly charged and continuously electrohydrated sodium nanoparticles-nanobatteries could probably take place in these specific experiments, while similar ball lightning clouds consisting of the negatively charged sodium-, calcium-, iron-, aluminium-, or carbon-based nanoparticles spontaneously converted into metal/air or carbon/air nanobatteries, could be generated by both the pulse electrolysis and arc evaporation of carbon/metal cathodes in the earlier similar experiments $[1,2]$.

ones, on the charged surface of such particles or surfaceintegrated structures which are in contact with any potential polar gas phase reactants.

In such cases, electrostatic charges (solvated ions or electrons), either adsorbed by these nanoparticles/nanostructures or induced on their surface by external fields, could probably act as local catalysts of various heterophase reactions.
Of course, the successive process of the charge-catalyzed predominantly water vapour induced electrochemical oxidation can take place in a humid atmosphere not only in relation to highly charged nano- or micrometer-sized combustible aerosol particles or substrate-integrated structures, but also in relation to certain highly charged macroscopic objects, for example, such as the corona-forming highly porous rusty iron/carbon-based electrodes, where a 


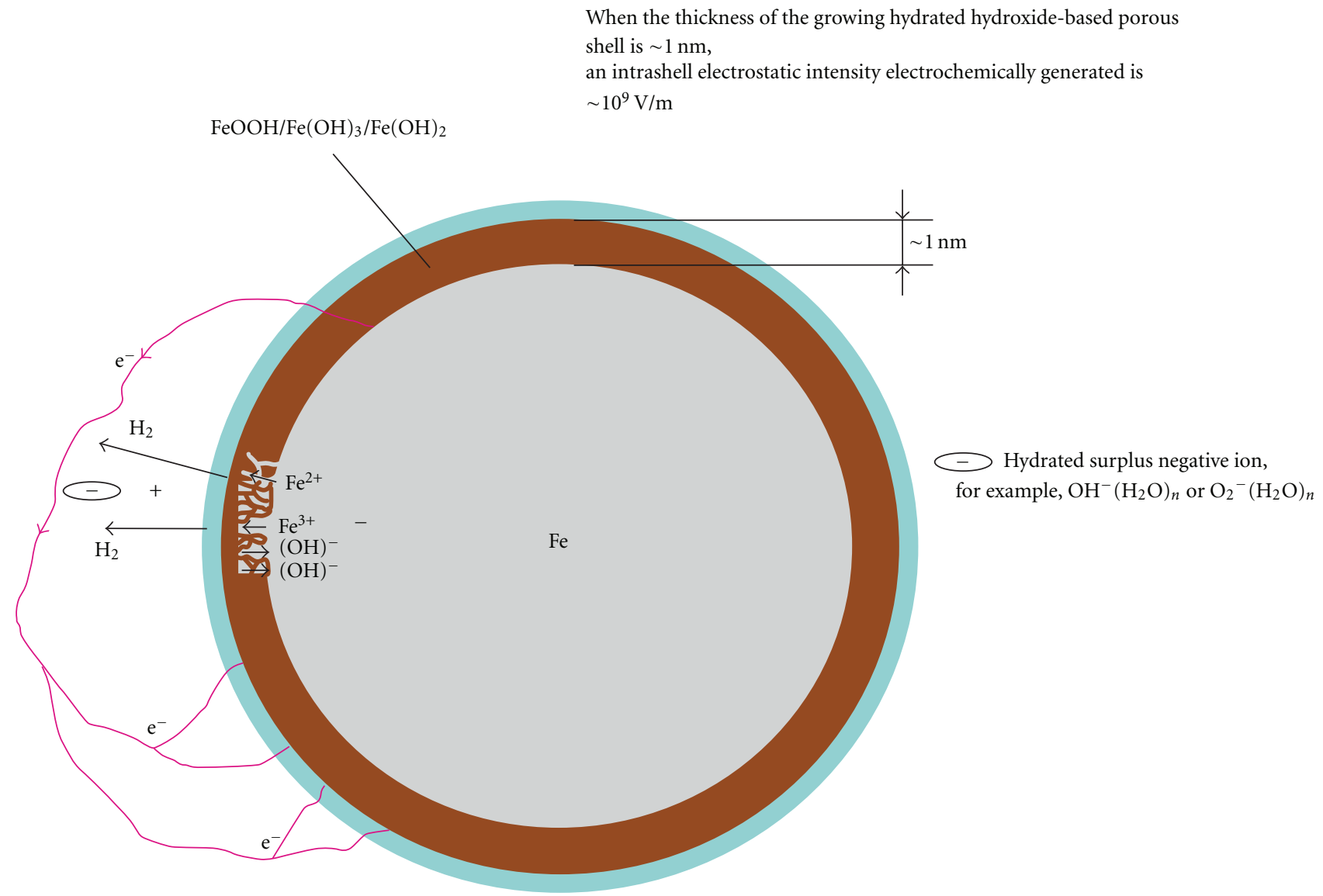

\footnotetext{
Dynamic layer of the absorbed molecules of water vapor

Dynamic porous layer/shell of the hydrated hydroxide electrolytes

An oxidizable core of nanoparticle
}

FIGURE 14: In humid air, negatively charged iron metal nanoparticles are continuously re-electrohydrated by surrounding molecules of water vapour, and so they can be spontaneously transformed into the iron/air core-shell nanobatteries, which are periodically short-circuited by the intraparticle electron emission breakdowns.

secondary electrospraying effect, which we earlier named "field-assisted metal dusting corrosion", could be caused by a local charge-catalyzed water vapour-induced oxidation of both carbon and iron metal components of corona-forming corrodible electrodes, with an additional local generation of water gas. In this case, the charge-dipole attraction between the charged porous surface of the steel or cast iron based high-voltage electrodes and the surrounding molecules of water vapour could play the role of an electrostatic water pump to transport and accelerate the water vapour polar molecules, but not the oxygen gas molecules, towards the charged oxidizable surface of the high-voltage electrodes.

Normally, uncharged pure carbon based nanomaterials, for example soot nanoparticles are quite inert in humid air. However, in view of the aforesaid it seems that highly charged carbon based nanoparticles can be much more reactive.
One of the possible direct reactions of the surrounding electrostatically accelerated molecules of water vapour with the charged carbon based aerosol nanoparticle can be the socalled water gas endothermic reaction

$$
\mathrm{C}_{\text {(nanoparticle) }}+\mathrm{H}_{2} \mathrm{O}_{\text {(vapour) }}=\mathrm{CO}+\mathrm{H}_{2}+131.3 \mathrm{~kJ} / \mathrm{mol} \text {. }
$$

Though this reaction is able to generate a combination of the two high-calorific fuel gases, this first stage of process of the water vapour induced oxidation of the carbon-based nanoparticle absorbs a lot of heat.

Alternative reactions of the charged carbon nanoparticle with the non-polar and, accordingly, electrostatically nonaccelerated molecules of ambient oxygen gas are exothermal reactions:

$$
2 \mathrm{C}_{(\text {nanoparticle })}+\mathrm{O}_{2}=2 \mathrm{CO}-221 \mathrm{~kJ} / \mathrm{mol}
$$




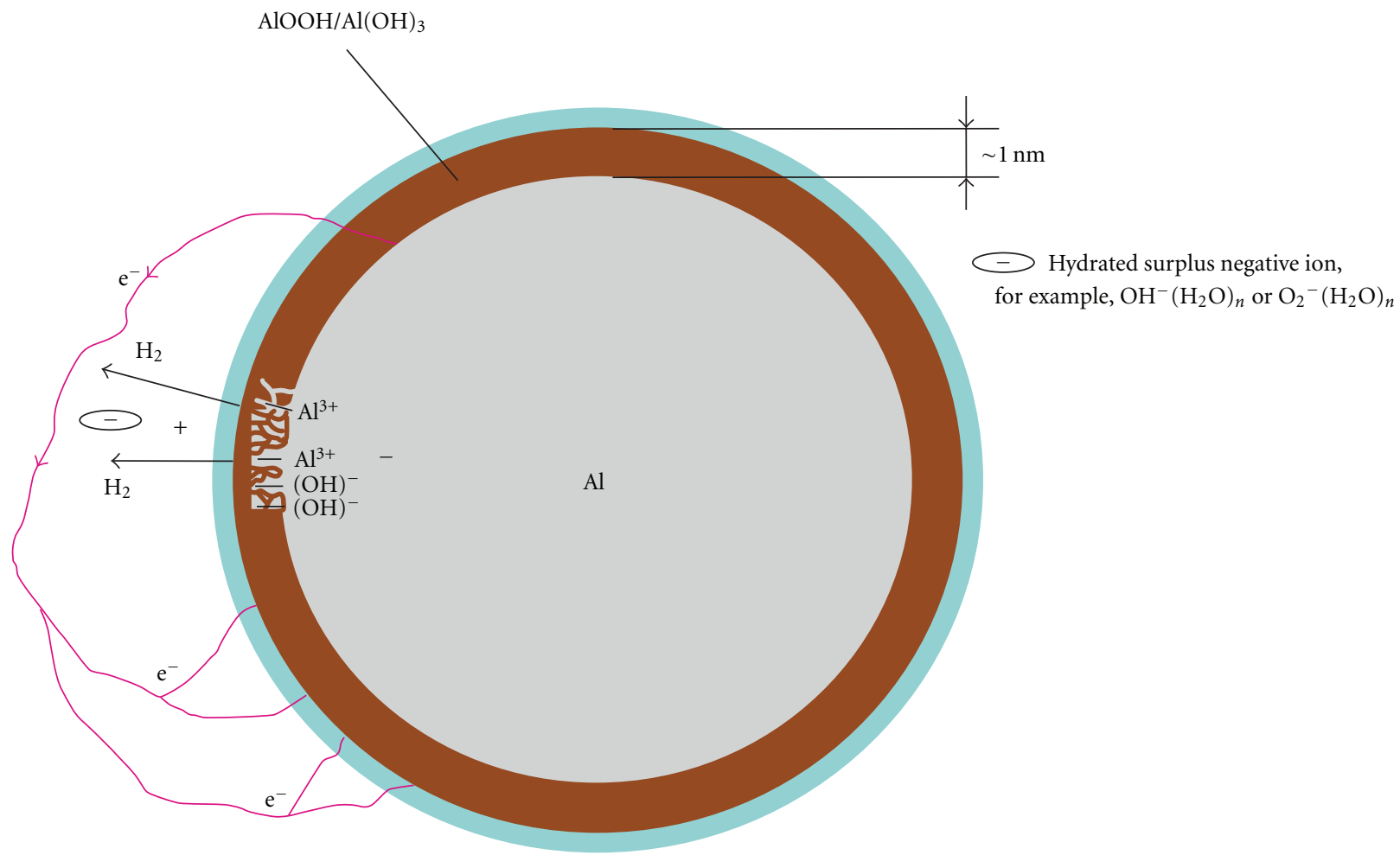

Dynamic layer of the absorbed molecules of water vapor Dynamic porous layer/shell of the hydrated hydroxide electrolytes An oxidizable core of nanoparticle

FIGURE 15: Similarly, in humid air, negatively charged aluminium nanoparticles can repeatedly be electrohydrated by surrounding molecules of water vapour, and so they can be spontaneously transformed into the aluminium/air core-shell nanobatteries, which are periodically short-circuited by the intraparticle electron emission breakdowns.

with carbon monoxide as the predominant product at a temperature above $800^{\circ} \mathrm{C}$, and

$$
\mathrm{C}_{(\text {nanoparticle })}+\mathrm{O}_{2}=\mathrm{CO}_{2}-393.5 \mathrm{~kJ} / \mathrm{mol}
$$

with carbon dioxide as the predominant product at a temperature $\sim 600-700^{\circ} \mathrm{C}$.

Intense electrostatic hydration of the oxygen gas preoxidized carbon based nanoparticles by the surrounding polar molecules of water vapour will contribute both to a temporary cooling of these red hot nanoparticles and to a pulse evolution of the secondary inflammable gases, that is, hydrogen gas and carbon monoxide gas. In ambient humid air, the heat released from subsequent auto-ignition of these high-calorific fuel gases

$$
\begin{aligned}
2 \mathrm{H}_{2 \text { (gas) }}+\mathrm{O}_{2 \text { (gas) }} & =2 \mathrm{H}_{2} \mathrm{O}_{(\text {vapour })}-484 \mathrm{~kJ} / \mathrm{mol} \\
\mathrm{CO}+\mathrm{H}_{2} \mathrm{O}_{(\text {vapour })} & =\mathrm{CO}_{2}+\mathrm{H}_{2}-41.2 \mathrm{~kJ} / \mathrm{mol} \\
2 \mathrm{CO}+\mathrm{O}_{2} & =2 \mathrm{CO}_{2}-566 \mathrm{~kJ} / \mathrm{mol}
\end{aligned}
$$

is again able to dehydrate and make the charged carbon based nanoparticles red-hot. This flame dehydration is able to restart the relatively high-temperature process of oxygen gas-induced oxidation of these electrostatically charged carbon-based nanoparticles. The high heat emission of these nanoparticles and their relatively low reactivity in normal humid air will again contribute to the fast cooling of these nanoparticles with restarting of their electrostatic hydration and their relatively low-temperature water vapourinduced oxidation. Thus, such a prolonged self-oscillating thermocycling process of the fast alternation of oxygen gas and water vapour-induced oxidation of the charged soot nanoparticles can probably take place in humid air.

Probably, pure water-based dynamic shells, consisting only of the water vapour molecules, electrostatically attacking the surface of the charged soot nanoparticles, are not able to directly convert these charged carbon nanoparticles into short-circuited carbon/air nanobatteries. The electroadsorbed dynamic layers of the pure superheated water are still a weak surface electrolyte to efficiently transport the ionized 
The simplest two-particle aerosol nanoaggregate can, for example, consist of:

(a) an oxidable metal nanoparticle, for example, such as ana iron, or aluminium, or tungsten, or molybdenium, or silicon nanoparticle, (b) a carbon /soot nanoparticle. and

(c) a hydrated oxide-hydroxide/hydroxide boundary layer (green). in humid air, the uncharged oxidizable metal or metalloid nanoparticle (yellow) is only slightly

covered by a hydrated oxide-hydroxide/hydroxide based shell (green), consisting of such components as, for example,

$\mathrm{FeOOH}$, or $\mathrm{AlOOH}$, or $\mathrm{H}_{2} \mathrm{WO}_{4}$, or $\mathrm{H}_{2} \mathrm{MoO}_{4}$, or $\mathrm{H}_{2} \mathrm{SiO}_{3}$.

If this metal/carbon nanoaggregate is uncharged with an additional charge, that is, if $Q=0(\mathrm{C})$, a surface hydration of the nanoaggregate by water vapor molecules

from ambient air is minimum, and the process of an electrochemical charge separation within such a neutral nanoaggregate-nanobattery is weekly marked.

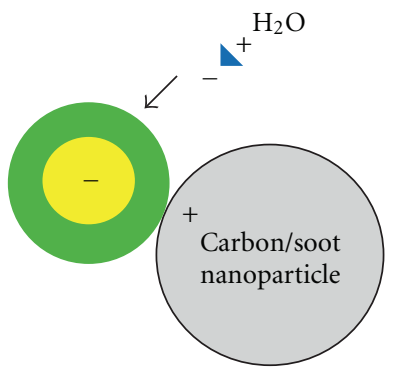

$Q=0(\mathrm{C})$

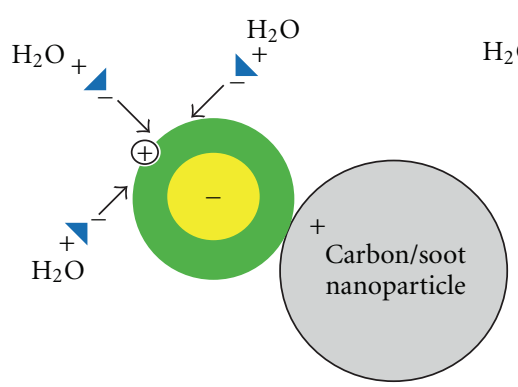

$Q=+1.6 \cdot 10^{-19}(\mathrm{C})$

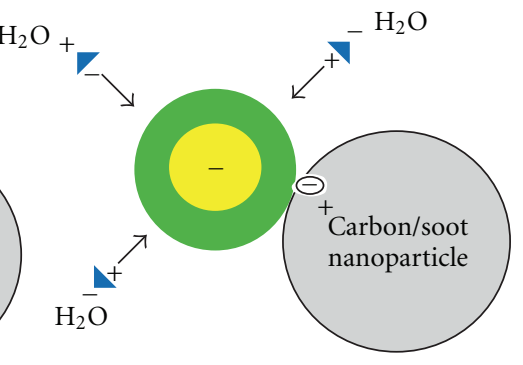

$Q=-1.6 \cdot 10^{-19}(\mathrm{C})$

In an alternative case, if a metal/carbon nanoaggregate is charged, an electrostatic hydration of such a charged nanoaggregate by water vapor molecules from ambient air may be substantial due to a charge-dipole attracting between the charged nanoaggregate and surrounding polar molecules of water vapor. Thus, processes of the electrochemical oxidation of the metal nanocomponent and internal electrochemical redistribution of charges within the charged aerosol nanoaggregate-nanobattery can be significantly activated by its electrostatic hydration.

This statin recalls the filling of a dry accumulator-charged aerosol metal/carbon/air nanoaggregate-nanobattery with a liqufied electrolyte superheated electrostatically accelerated molecules of water vapor that are continuously or periodically absorbed on the surface of the charged nanoaggregate-nanobattery. The charged metal/carbon/air nanoaggregates-nanobatteries are evolving hydrogen gas and are periodically short-circuited by an electron emission from a metal anode to a carbon cathode of these aerosol nanobatteries.

FIGURE 16: Charged aggregated aerosol nanocomposites, for example the aggregated aerosol nanobatteries or aggregated aerosol nanothermites are continuously electrostatically rehydrated in humid air. This can result in a periodic transformation of the metal oxide based dielectric or semiconductor interphase barriers into the metal hydroxide based electrolyte interphase barriers, with the corresponding periodic reactivation of the electrochemical and electron emission discharge processes in the aggregated aerosol nanocomposites.

oxidizing species into the oxidizable surface of the carbon core.

It is possible that additional strong electrolyte surface impurities, such as sodium hydroxide/sodium carbonate or potassium hydroxide/potassium carbonate or calcium hydroxide/calcium carbonate or barium hydroxide/barium carbonate aerosol impurities, cocondensed and/or coaggregated along with soot nanoparticles, could contribute to a conversion of such molten salt electrolyte aggregated charged soot nanoparticles into the short-circuited carbon/air aerosol nanobatteries in humid air.

The intense electrostatic acceleration and corresponding substantial activation of polar gas molecules, for example, such as molecules of hydrogen peroxide, nitric acid, nitrogen monoxide, carbon monoxide, ozone, sulphuric acid, ammonia, hydrogen sulphide, hydrochloric acid, as well as many organic polar molecules, which take place in immediate proximity to naturally or artificially electrostatically charged oxidizable nanoobjects, can perhaps be successfully used in many nanotechnological applications, for example in those such as heterophase catalysis, heterophase copolymerization with the participation of polar monomers (e.g., methyl methacrylate, styrene, etc.) on the surface of highly charged nanoscale catalysts of polymerization, in exhaust systems with the purpose of a secondary combustion of soot nanoparticles artificially highly precharged, either with the help of a high-frequency corona discharge, or by bipolar direct current corona discharge, either by contact, or microwave or plasma charging. Such electrostatic or electrodynamic catalysis of the oxidation/combustion processes with an intense high-frequency bipolar charging of solid or liquid fuel particles in engines or power generating plants could also be of practical interest.

Generally speaking, similar intense charge-dipole attacks of electrostatically extra accelerated polar gas molecules, directed against adjacent highly charged nanoobjects, can probably be a widespread phenomenon in nature. In particular, such electrostatic oxidative attacks of the polar gas molecules (e.g., blood nano-bubble contained polar gas molecules of hydrogen peroxide vapour, water vapour, hydrogen sulphide, hydrochloric acid, nitrogen monoxide, carbon monoxide etc.), directed against naturally or artificially, electrostatically or electrodynamically charged nanobio-objects, for example, such as lipoproteins open to injury by oxidation or DNA's open to injury by mutations (that can be generated due to possible high-energy collisions DNA's based biopolymers with the surrounding charge-dipole extra accelerated epithermal polar gas molecules), can probably play the important role in both natural biological processes and potential biomedical uses.

So, we assume that a ball lightning substance consists of short-circuited aerosol nanobatteries. Also, we assume that electrostatic oxidative hydration of highly charged combustible aerosol nanoparticles can substantially change the rate and mechanism their atmospheric oxidation in humid air, converting such nanoparticles into reductant/air 

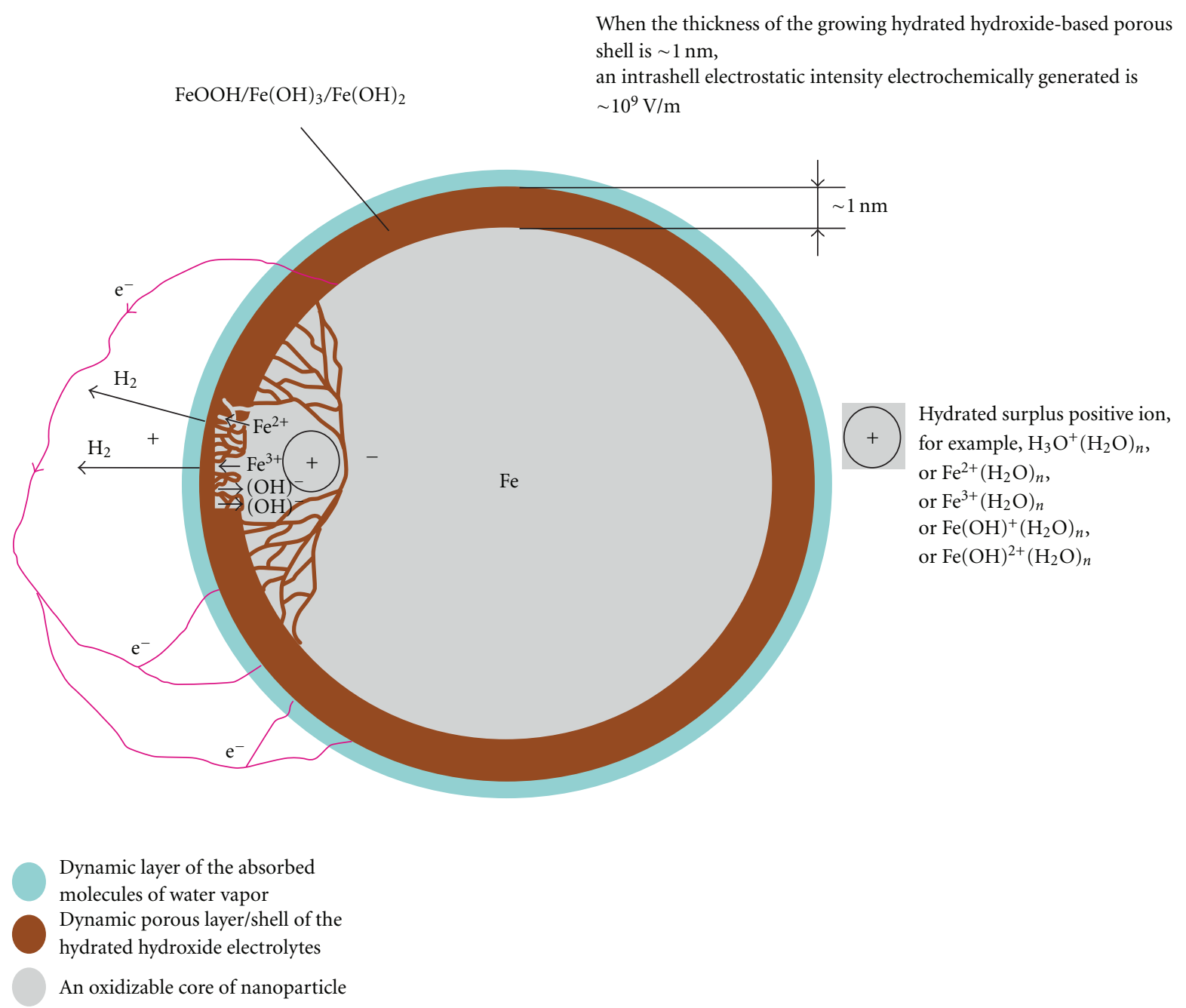

FIGURE 17: Positively charged iron nanoparticles can equally be continuously re-electrohydrated by surrounding molecules of water vapour, and so they can be transformed into the iron/air core-shell nanobatteries, periodically short-circuited by the intra-particle electron emission breakdowns.

aerosol nanobatteries, periodically short-circuited by internal electron emission breakdowns. At the same time, we would like to emphasize that such reductant/air nanobatteries, that can be spontaneously generated from highly charged combustible nanoparticles in humid air, could be an electrogenerating solid fuel aerosol component for only one type of ball lightning, that is, for the electrochemical nanobatteries based ball lightning that use mainly external gas oxidants, water wapour and oxygen.

To avoid misunderstanding and/or excessive generalizations concerning a possible key role of atmospheric water vapour in a life of any types of the nanobattery based lightning balls, we would also like to emphasize that certain hypothetic sorts of the short-circuited aerosol nanobatteries can contain their own, solid or liquid phase, compact oxidant nanocomponents. Such completely self-contained electrochemical aerosol nanobatteries can exceptionally use the internal condensed fuel and oxidant nanocomponents, either aggregated or core/shell structured [20]. Thus, an influence of external atmospheric gas oxidants on the internal redox and discharge processes in such completely self-contained aerosol nanobatteries could be negligible.

Moreover, it is clear that not only electrochemical processes inside composite nanoparticles can contribute to their spontaneous transformation into short-circuited nanobatteries, constituting an electrogenerating aerosol substance of ball lightning. For example, it would be reasonable to assume that highly exothermic self-propagating processes of flameless combustion, that is, self-propagating hightemperature synthesis or SHS, could frequently arise inside either composite aerosol micro- or nanoaggregates or inside composite aerosol micro- or nanodroplets, containing at least two adjacent condensed heterogeneous reactants. Probably, such highly exothermic SHS processes could proceed in a self-oscillating mode inside composite aerosol particles, contributing to a partial or total melting of constituting them micro- or nanoreactants, also contributing to intra-particle formation of new phases in the form of intermetallic/ceramic 


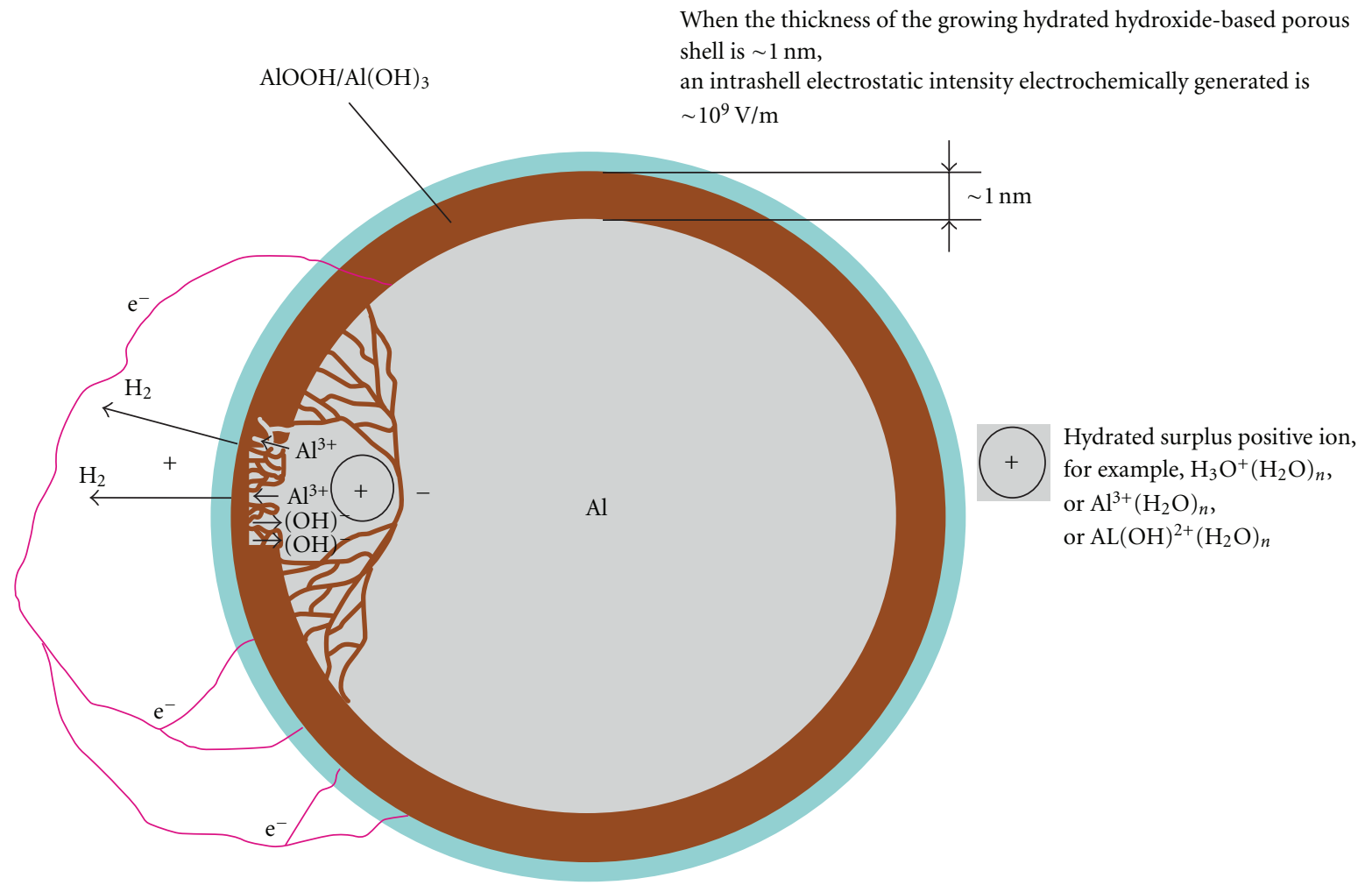

FIGURE 18: Positively charged aluminium nanoparticles can equally be electrohydrated by surrounding molecules of water vapour, and so they can be transformed into the aluminium/air core-shell nanobatteries, periodically short-circuited by the intra-particle electron emission breakdowns.

reaction products, and also contributing to an intense interphase charge separation. Such interphase charge separation inside the composite aerosol particles-microscopic aerosol SHS reactors-could be caused by several reasons, for example, both thermoelectric and thermionic interphase processes due to extremely high temperature gradients and extremely high rates of heating $\left(\sim 10^{3}\right.$ to $\left.10^{6} \mathrm{~K} / \mathrm{sec}\right)$, that generally take place in a SHS combustion wave. The intraparticle processes of the self-propagating high-temperature synthesis can probably generate a very intense, thermoelectrically and thermionically caused, interphase charge separation inside the composite aerosol/aerogel nanoaggregates or nanodroplets, which will automatically convert such composite nanoparticles into thermoelectric nanobatteries or thermionic nanoconverters. Such thermoelectric nanobatteries and thermionic nanoconverters, being formed from the intra-particle SHS-reacting condensed aerosol nanoreactants, and being periodically short-circuited by intra-particle/surface electron emission breakdowns, could, in principle, be another type of an electrogenerating solid fuel aerosol substance for a nanobattery based ball lightning. In this case, inside such "thermoelectric" or "thermionic" nanobattery based ball lightning, an influence of external gas oxidants on the intra-particle SHS and electric processes could also be substantially minimized due to passivating oxide shells growing on outer surface of such hightemperature thermoelectric or thermionic nanobatteries.

It is clear, when highly exothermic SHS processes take place inside the heterogeneous composite aggregated aerosol particles/droplets, a formation of the passivating dense oxidelayers on the outer surface of these aerosol composite particles can contribute to a vital protection of such internally burning hot particles from their external oxidation by atmospheric gas oxidants.

Probably, a lot of different pyrotechnic aerosol nanocomposites, spontaneously formed from combustible aerosol nanoparticles in a local reducing or inert atmosphere, could be transformed into the short-circuited high-temperature 
thermoelectric/thermionic aerosol nanobatteries through the intra-particle highly exothermic SHS processes. In particular, bimetallic composite particles consisting, for example, of aluminium metal and nickel metal could form selfcontained micro- or nanoaggregates/nanodroplets, externally covered with a dense alumina shell. In such $\mathrm{Ni} / \mathrm{Al}$ nanocomposites, highly exothermic intra-particle SHS reactions between metallic aluminium and nickel will synthesize new numerous intermetallic phases of nickel aluminides. The intra-particle high-temperature processes of flameless synthesis of nickel aluminides can be correspondingly accompanied with synchronous formation of numerous interphase nanothermocouples, such as the nickel/aluminium, or nickel/nickel aluminide, or aluminium/nickel aluminide nanothermocouples and so forth.

Similarly, composite aerosol particles, consisting, for example, of titanium metal and soot carbon nanoparticles-nanoreactants, or titanium metal and silicon nanoparticles-nanoreactants, could form micro- or nanoaggregates/droplets, externally covered with either a porous soot carbon shell or with a dense silica shell, inside which highly exothermic intra-particle liquid/solid SHS reactions between titanium and carbon, or between titanium and silicon, will synthesize titanium carbides reaction products, such as $\mathrm{TiC}$ (with enthalpy of formation $\sim 186 \mathrm{~kJ} / \mathrm{mol}$ ), or titanium silicides, such as TiSi (with enthalpy of formation $\sim 73 \mathrm{~kJ} / \mathrm{mol}$ ).

Similarly, numerous nanothermocouples, such as titanium/silicon or titanium/titanium carbide, or titanium/ titanium silicide nanothermocouples and so forth, could be formed, and then be periodically short-circuited by the electron emission breakdowns, inside the intra particle combustion waves.

A lot of aerosol nanoreactants, such as nanoparticles of aluminium, titanium, silicon, tantalum, zirconium, calcium, boron, carbon, platinum, palladium, molybdenum, tungsten, zinc, sulfur, selenium and so forth, are able to mutual highly exothermic SHS reactions, accompanied with formation of intermetallic and/or ceramic nanocompositions. Being formed from the arc-, or microwave-, or laserproduced high-temperature condensation aerosol nanoreagents in a local reducing or inert atmosphere, carbides of tantalum, or zirconium, or vanadium, or titanium, or niobium; silicides of platinum, or palladium, or molybdenum, or tungsten, or zirconium; aluminides of nickel, or cobalt, or calcium, or platinum, or palladium, or iron; borides of tantalum, or zirconium, or vanadium, or titanium, or niobium, or molybdenum; sulphides of magnesium, or zinc, or molybdenum, or tungsten; all these flameless combustion products could be primary products of the particularly highly exothermic intra particle SHS reactions.

Consequently, a lot of the bi-component or multi-component composite aerosol particles, consisting of widespread mutually reacting condensed substances, could be excellent candidates to spontaneously form the short-circuited thermoelectric/thermionic aerosol nanobatteries in the highly exothermic intra-particle SHS processes. In nature, a lightning induced, total or subtotal high-temperature gas phase reduction of ordinary aluminosilicate/clay minerals by carbon or hydrogen, that can be generated by a synchronous pyrolysis of adjacent organic substances, could form unipolar charged, bi- or polymetal composite aerosol nanoparticles, that is, burning hot nanoaggregates or nanodroplets that contain the reacting condensed SHS nanocomponets. Such aluminosilicate-derived, hot aerosol composite nanoparticles can consist, for example, of the mutually highly reactive Al-Ca-Si-Fe-Ti metals, partially mixed with metal oxide nanocomponents and partially protected from atmospheric gas phase oxidation by outer mixed metal oxide shells. Inside such clay-derived polymetal nanocomposites, numerous thermoelectric and thermionic nanobatteries can probably be formed from the high-temperature interphase contacts, due to huge local temperature gradients, that continuously arise in intra-particle self-propagating combustion waves.

Probably, even in many other aspects relatively well studied thermite based nanocomposites, particularly, socalled aerosol nanothermites, consisting, for example, of the $\mathrm{Al} / \mathrm{Fe}_{3} \mathrm{O}_{4}$ or $\mathrm{Al} / \mathrm{CuO}$ nanomixtures with some overstoichiometric excess of aluminium metal, also could spontaneously form burning ball lightning like clouds, containing the short-circuited thermoelectric and/or thermionic aerosol nanobatteries, that can be based on the spontaneously formed $\mathrm{Al} / \mathrm{Fe}-$, or $\mathrm{Al} / \mathrm{FeO}-, \mathrm{Al} / \mathrm{Fe}_{3} \mathrm{O}_{4}{ }^{-}$, or $\mathrm{Al} / \mathrm{Cu}-$, or $\mathrm{Al} / \mathrm{Cu}_{2} \mathrm{O}$-nanocontacts/nanothermocouples, and that can be periodically shortcircuited by internal electron emission breakdowns. During the highly exothermic SHS reactions, self-propagating intra-particle waves of thermionic electron emission short-circuits, arising on the developed surface of the thermoelectric/thermionic aerosol nanobatteries, can probably be closely coupled with the intra-particle selfpropagating combustion waves.

Inside a cloud that consist of trillions of such flameless burning composite nanoparticles-SHS nanoreactorsperiodic intra-particle electron emission breakdowns could generate powerful total microwave radiation, contributing to interparticle electromagnetic dipole-dipole attraction that will compress this luminous ball lightning cloud.

Thus, as one can see, the preliminary described effect of the preferential water vapour induced oxidation of the highly charged combustible aerosol nanoparticles in humid air can be only one of many important, interesting, and mutually complementary phenomena, which careful study could help us to understand the mysterious nature of ball lightning.

\section{References}

[1] A. I. Egorov, S. I. Stepanov, and G. D. Shabanov, "Laboratory demonstration of ball lightning," Physics-Uspekhi, vol. 47, no. 1, pp. 99-101, 2004.

[2] A. Versteegh, K. Behringer, U. Fantz, G. Fussmann, B. Jüttner, and S. Noack, "Long-living plasmoids from an atmospheric water discharge," Plasma Sources Science and Technology, vol. 17, no. 2, Article ID 024014, 2008.

[3] G. S. Paiva, A. C. Pavão, E. Alpes de Vasconcelos, O. Mendes, and E. Felisberto da Silva Jr., "Production of ball-lightninglike luminous balls by electrical discharges in silicon," Physical Review Letters, vol. 98, no. 4, Article ID 048501, 2007. 
[4] Y. Sakawa, K. Sugiyama, T. Tanabe, and R. More, "Fireball generation in a water discharge," Plasma and Fusion Research: Rapid Communications, vol. 1, article 039, 2006.

[5] J. B. A. Mitchell, J. L. Legarrec, M. Sztucki, T. Narayanan, V. Dikhtyar, and E. Jerby, "Evidence for nanoparticles in microwave-generated fireballs observed by synchrotron X-ray scattering," Physical Review Letters, vol. 100, no. 6, Article ID 065001, 2008.

[6] E. Jerby, A. Golts, Y. Shamir et al., "Nanoparticle plasma ejected directly from solid copper by localized microwaves," Applied Physics Letters, vol. 95, no. 19, Article ID 191501, 2009.

[7] N. Hayashi, H. Satomi, T. Kajiwara, and T. Tanabe, "Properties of ball lightning generated by a pulsed discharge on surface of an electrolyte in the atmosphere," IEEJ Transactions on Electrical and Electronic Engineering, vol. 3, no. 6, pp. 731-733, 2008.

[8] I. Stakhanov, About Physical Nature of Ball Lightning, Energoatomizdat, Moscow, Russia, 1985.

[9] A. I. Grigor'ev, Ball Lightning, YarGU Yaroslavl, 2006.

[10] C. Chun, J. Mumford, and T. Ramanarayanan, "On the mechanism of metal dusting corrosion," http://www.electrochem.org/dl/ma/201/pdfs/1111.pdf.

[11] R. Jones and K. Baumert, "Metal dusting - an overview of current literature," NACE International, Corrosion, paper no. 01372, 2001.

[12] H. J. De Bruyn, E. Edwin, and S. Brendryen, "Apparent influence of steam on metal dusting," NACE International, Corrosion, paper no. 01383, 2001.

[13] Z. Zeng and K. Natesan, "Relationship between the growth of carbon nanofilaments and metal dusting corrosion," Chemistry of Materials, vol. 17, no. 14, pp. 3794-3801, 2005.

[14] J. C. Nava Paz and H. J. Grabke, "Metal dusting," Oxidation of Metals, vol. 39, no. 5-6, pp. 437-456, 1993.

[15] C. M. Chun and T. A. Ramanarayanan, "Metal-dusting corrosion of low-chromium steels," Oxidation of Metals, vol. 62, no. 1-2, pp. 71-92, 2004.

[16] A. Al-Meshari and J. Little, "Oxidation of heat-resistant alloys," Oxidation of Metals, vol. 69, no. 1-2, pp. 109-118, 2008.

[17] K. D. Stephan, "Electrostatic charge bounds for ball lightning models," Physica Scripta, vol. 77, no. 3, Article ID 035504, 2008.

[18] D. J. Turner, "Ball lightning and other meteorological phenomena," Physics Report, vol. 293, no. 1, pp. 1-60, 1998.

[19] J. Abrahamson and J. Dinniss, "Ball lightning caused by oxidation of nanoparticle networks from normal lightning strikes on soil," Nature, vol. 403, no. 6769, pp. 519-521, 2000.

[20] O. Meshcheryakov, "Ball lightning-aerosol electrochemical power source or a cloud of batteries," Nanoscale Research Letters, vol. 2, no. 7, pp. 319-330, 2007.

[21] A. P. Grosvenor, B. A. Kobe, and N. S. McIntyre, "Activation energies for the oxidation of iron by oxygen gas and water vapour," Surface Science, vol. 574, no. 2-3, pp. 317-321, 2005.

[22] A. P. Grosvenor, B. A. Kobe, and N. S. McIntyre, "Studies of the oxidation of iron by water vapour using X-ray photoelectron spectroscopy and QUASES ${ }^{\mathrm{TM}}$," Surface Science, vol. 572, no. 23, pp. 217-227, 2004.

[23] A. P. Grosvenor, B. A. Kobe, and N. S. McIntyre, "Examination of the oxidation of iron by oxygen using X-ray photoelectron spectroscopy and QUASES ${ }^{\mathrm{TM}}$," Surface Science, vol. 565, no. 23, pp. 151-162, 2004.

[24] A. P. Grosvenor, B. A. Kobe, and N. S. McIntyre, "Studies of the oxidation of iron by air after being exposed to water vapour using angle-resolved X-ray photoelectron spectroscopy and
QUASES $^{\mathrm{TM}}$," Surface and Interface Analysis, vol. 36, no. 13, pp. 1637-1641, 2004.

[25] A. P. Grosvenor, B. A. Kobe, N. S. McIntyre, S. Tougaard, and W. N. Lennard, "Use of QUASES ${ }^{\mathrm{TM}} / \mathrm{XPS}$ measurements to determine the oxide composition and thickness on an iron substrate," Surface and Interface Analysis, vol. 36, no. 7, pp. 632-639, 2004.

[26] C. Govern, I. Spitsberg, and B. T. Hazel, "Protective layer for barrier coating for silicon-containing substrate and process for preparing same," US Patent Application 20060211241, 2006.

[27] D. E. Hagen and S. H. Suck Salk, "Entropy and enthalpy change in hydrated ion clusters," Chemical Physics, vol. 102, no. 3, pp. 459-466, 1986.

[28] T. Langer, D. Markus, F. Lienesch, and U. Maas, "Ignition of hydrogen/air mixtures by streamer discharges," in Proceedings of the European Combustion Meeting, 2009, http://www.combustion.org.uk/ECM_2009/P810066.pdf.

[29] N. Cabrera and N. F. Mott, "Theory of the oxidation of metals," Reports on Progress in Physics, vol. 12, no. 1, pp. 163$184,1949$.

[30] A. Fromhold, Theory of Metal Oxidation, North Holland, Amsterdam, 1976.

[31] G. Amato, "Silica-encapsulated efficient and stable Si quantum dots with high biocompatibility," Nanoscale Research Letters, vol. 5, no. 7, pp. 1156-1160, 2010.

[32] M. Graham, S. Ali, and M. Cohen, "Low temperature oxidation $\left(24^{\circ}\right.$ to $\left.200^{\circ} \mathrm{C}\right)$ and krypton adsorption studies on polycrystalline and single crystal iron surfaces," Journal of the Electrochemical Society, vol. 117, no. 4, pp. 513-516, 1970.

[33] D. A. Channing and M. J. Graham, "A study of iron oxidation processes by Mössbauer spectroscopy," Corrosion Science, vol. 12, no. 3, pp. 271-IN13, 1972.

[34] P. L. Kapitsa, “O priroda sharovoi molnii," Doklady Akademii Nauk SSSR, vol. 101, pp. 245-248, 1955.

[35] Y. H. Ohtsuki and H. Ofuruton, "Plasma fireballs formed by microwave interference in air," Nature, vol. 350, no. 6314, pp. 139-141, 1991.

[36] H. Ofuruton and Y. H. Ohtsuki, "Experimental research on ball lightning," Il Nuovo Cimento C, vol. 13, no. 4, pp. 761$768,1990$.

[37] H. Ofuruton, N. Kondo, M. Kamogawa, M. Aoki, and Y. H. Ohtsuki, "Experimental conditions for ball lightning creation by using air gap discharge embedded in a microwave field," Journal of Geophysical Research D, vol. 106, no. 12, pp. 1236712369, 2001.

[38] V. Dikhtyar and E. Jerby, "Fireball ejection from a molten hot spot to air by localized microwaves," Physical Review Letters, vol. 96, no. 4, Article ID 045002, 2006.

[39] M. Schoenitz, C. M. Chen, and E. L. Dreizin, "Oxidation of aluminum particles in the presence of water," Journal of Physical Chemistry B, vol. 113, no. 15, pp. 5136-5140, 2009.

[40] M. Tello and R. García, "Giant growth rate in nano-oxidation of p-silicon surfaces by using ethyl alcohol liquid bridges," Applied Physics Letters, vol. 83, no. 12, pp. 2339-2341, 2003.

[41] K. Bhavani and V. K. Vaidyan, "Effect of an electric field on the oxidation of aluminium at room temperature," Bulletin of Materials Science, vol. 2, no. 4, pp. 265-270, 1980. 

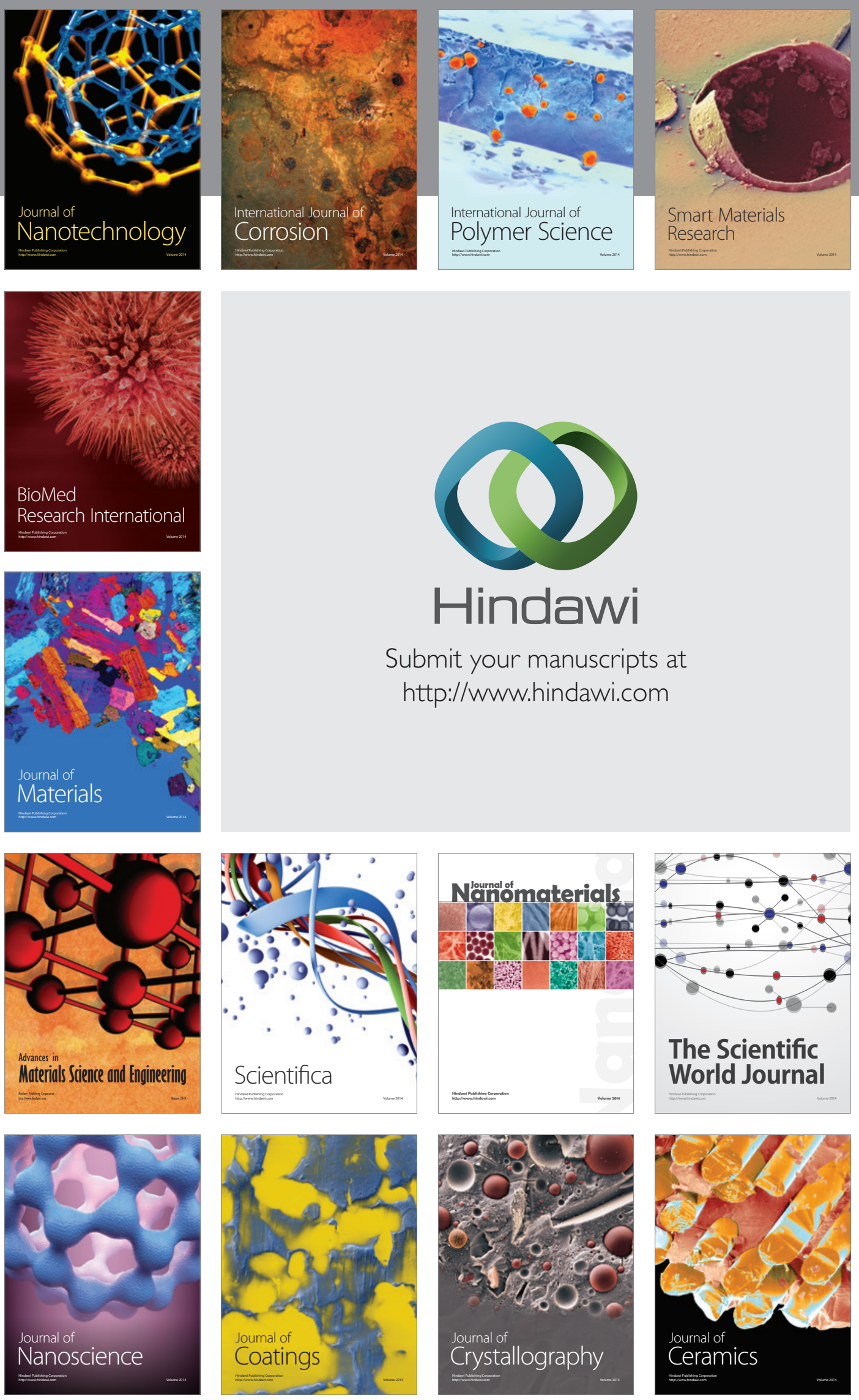

The Scientific World Journal

Submit your manuscripts at

http://www.hindawi.com

\section{World Journal}

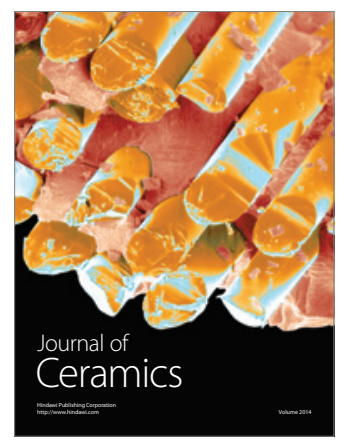

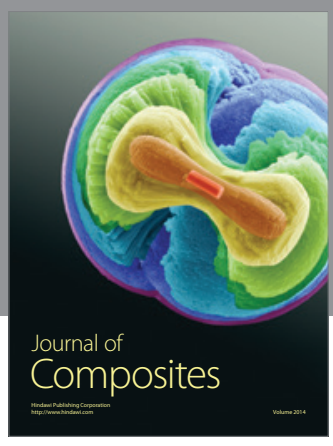
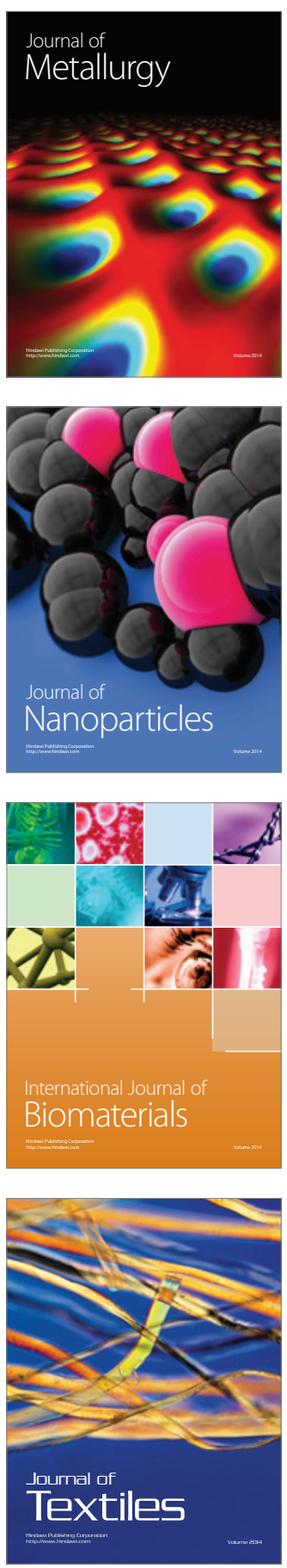